\title{
COMBINING TRANSCRANIAL ELECTRICAL STIMULATION WITH MAGNETIC RESONANCE IMAGING IN BEHAVIOURAL MEASUREMENTS IN HEALTH AND DISEASE
}

\author{
Dissertation \\ zur Erlangung des mathematisch-naturwissenschaftlichen Doktorgrades \\ "Doctor rerum naturalium" \\ Der Georg-August-Universität Göttingen
}

vorgelegt von

Catarina Saiote

aus Lissabon, Portugal

Göttingen, 2014 
Doctoral Thesis Committee:

Prof. Dr.rer. nat. Andrea Antal (Supervisor, First Referee)

Georg August University Göttingen

University Medical Center,

Department of Clinical Neurophysiology

Robert-Koch-Straße 40, 37075 Göttingen, Germany

\section{Prof. Dr. Michael Hörner}

Georg August University Göttingen

Coordinator MSc/PhD Neuroscience Program

International Max Planck Research School

European Neuroscience Institute

Grisebachstraße 5, 37077 Göttingen, Germany

\section{Prof. Dr. Florentin Wörgötter}

Georg-August-Universität Göttingen

Bernstein Center for Computational Neuroscience

Department for Computational Neuroscience

III Physikalisches Institut - Biophysik

Friedrich-Hund Platz 1, 37077 Göttingen, Germany

Date of submission: $30^{\text {th }}$ January, 2014

Date of examination: $31^{\text {st }}$ March, 2014 


\section{Statement of Originality}

I hereby declare that this thesis is my own work and has been written independently with no other sources and aids than quoted in the text, contributions, references and acknowledgements.

Göttingen, 30th January 2014

Catarina Saiote Ferreira Leite 


\section{Table of Contents}

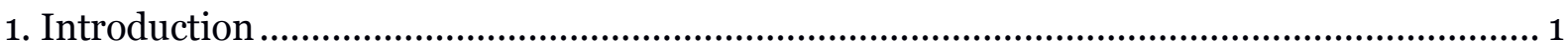

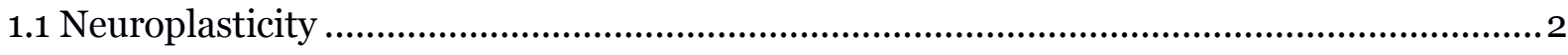

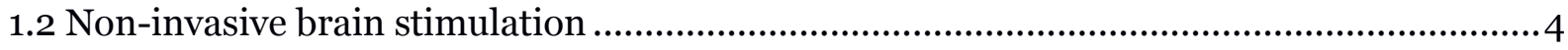

1.2.1 Transcranial direct current stimulation …............................................................. 5

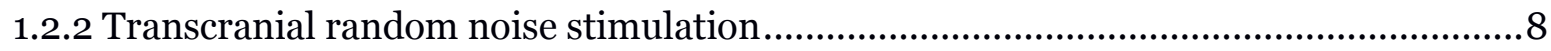

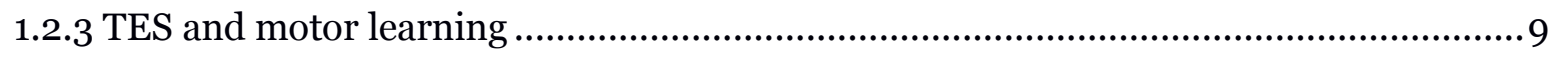

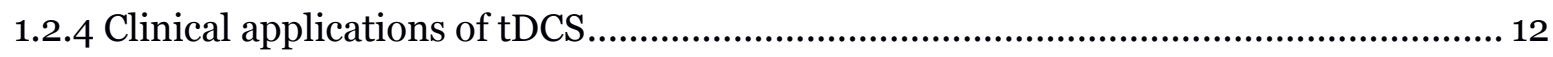

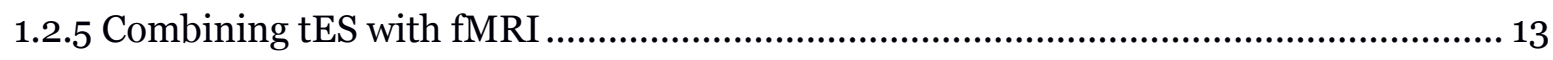

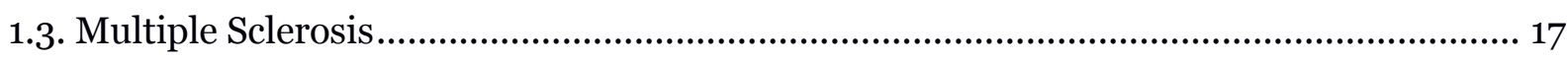

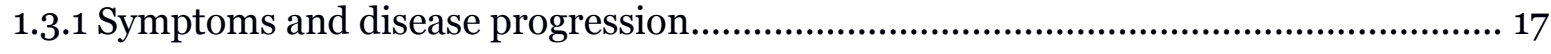

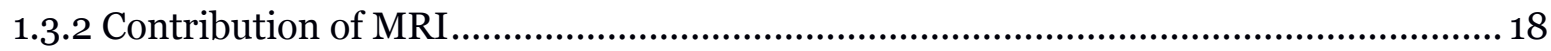

1.3.3. Fatigue in Multiple Sclerosis ................................................................................. 19

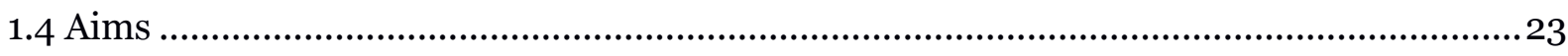

2. Impact of tDCS and tRNS on visuomotor learning behaviour and associated brain activity

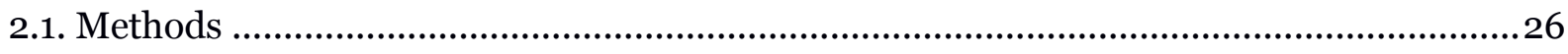

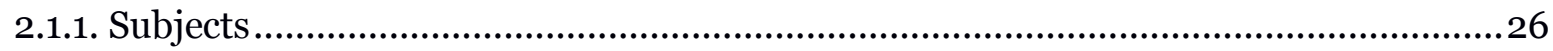

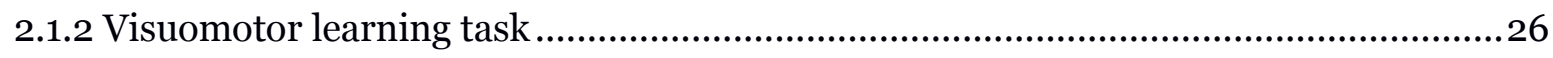

2.1.3. Experimental design....................................................................................... 27

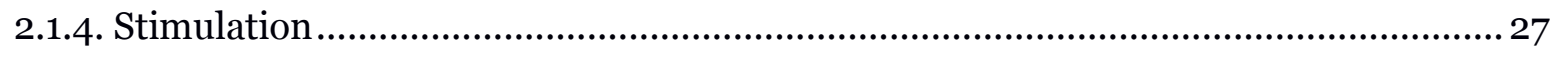

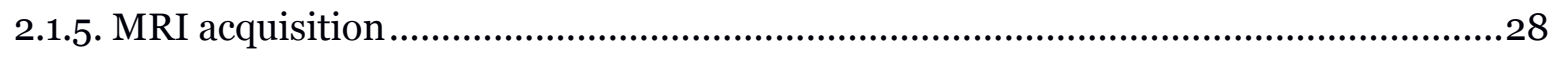

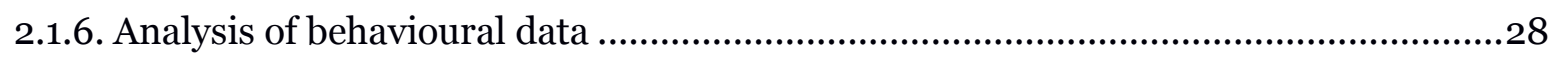

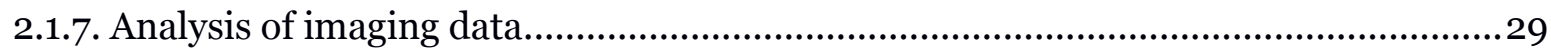




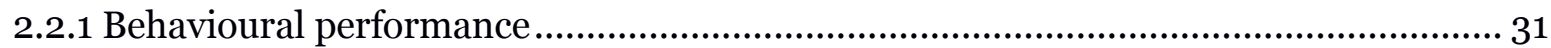

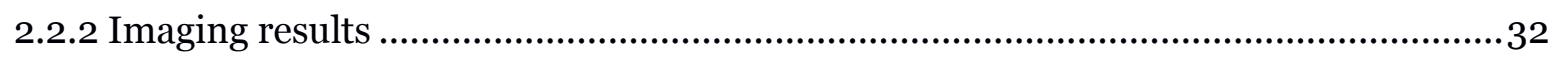

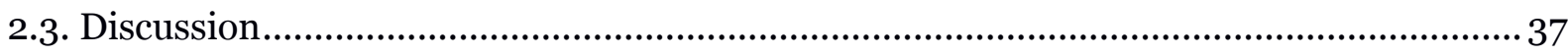

2.3.1 General learning and associated brain activity .................................................... 37

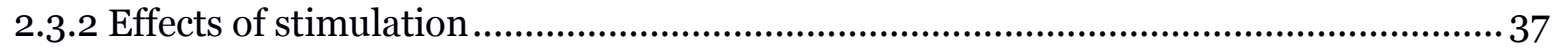

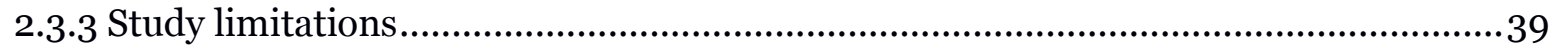

3. Project 2 - Improvement of fatigue symptoms in patients with multiple sclerosis with

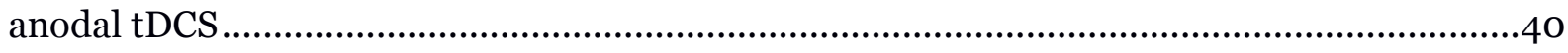

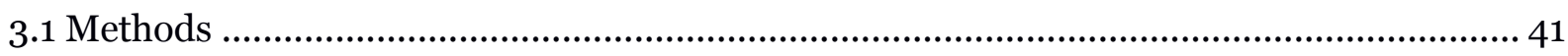

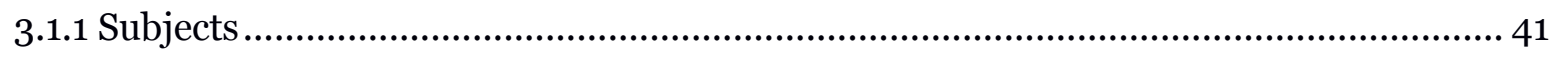

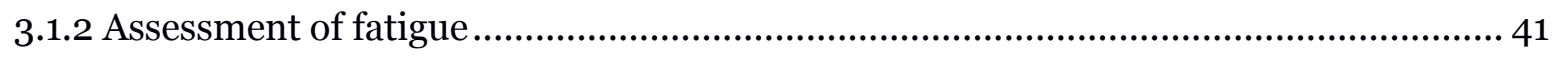

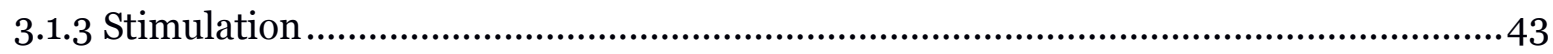

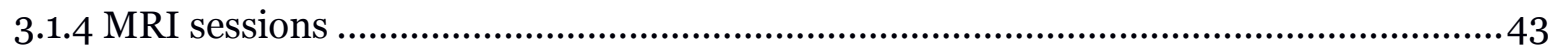

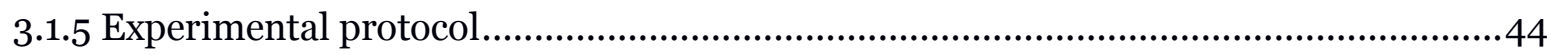

3.1.6 Neuron-specific enolase (NSE) ....................................................................... 45

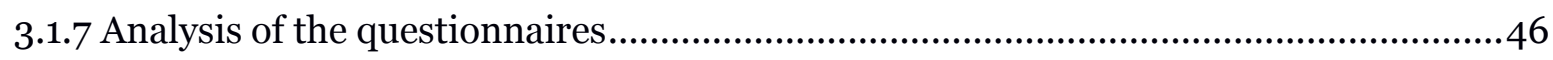

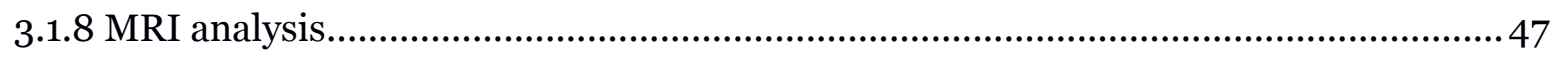

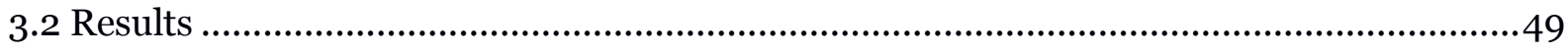

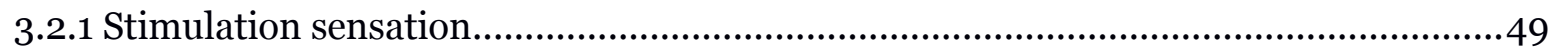

3.2.2 Neuron-specific enolase ..................................................................................49

3.2.3 Correlation of fatigue scales at baseline ................................................................50

3.2.4 Subjective changes of fatigue .............................................................................5

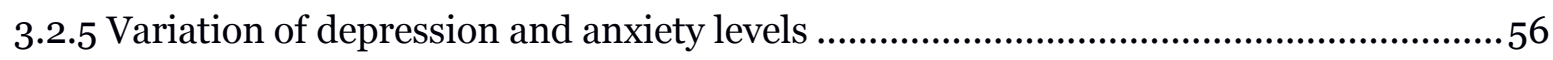

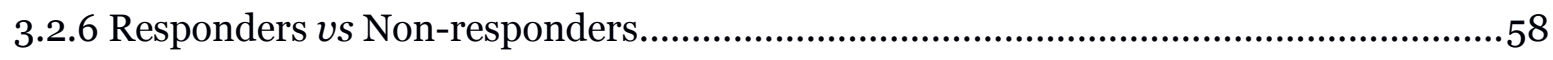




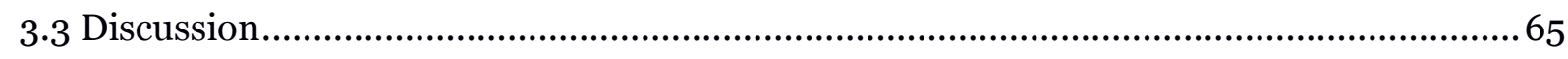

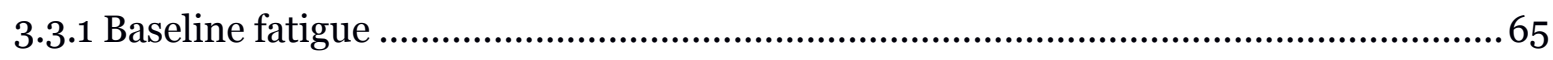

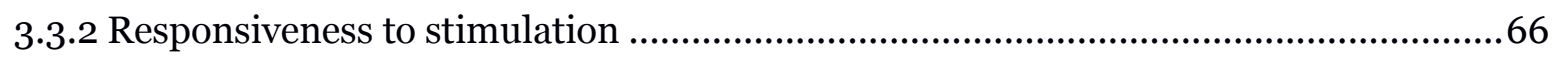

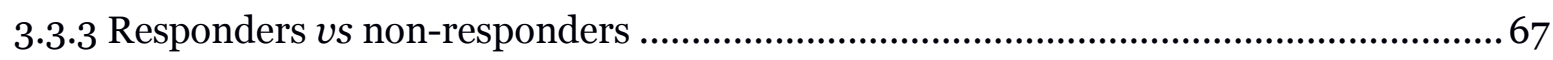

3.3.4 Fatigue scales results and limitations.................................................................68

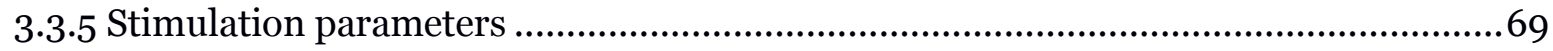

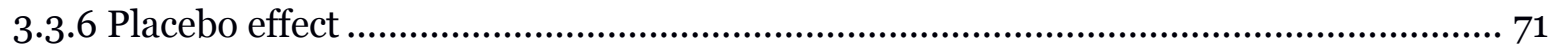

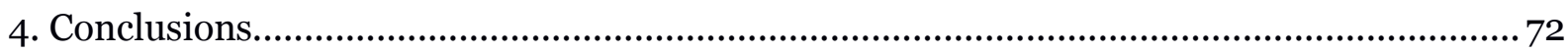

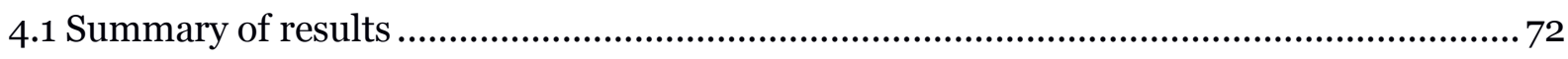

4.2 Final conclusions and future directions .................................................................. 73

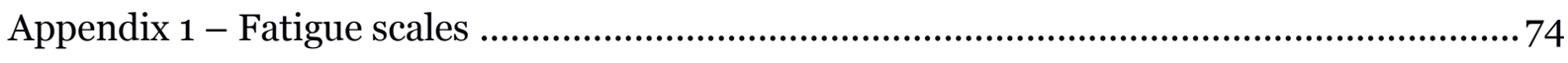

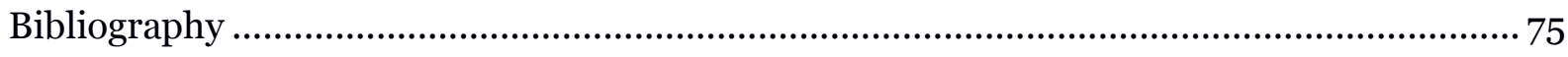

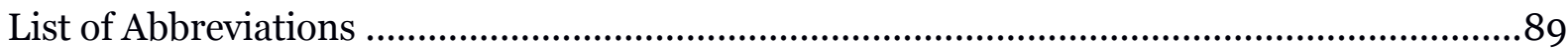

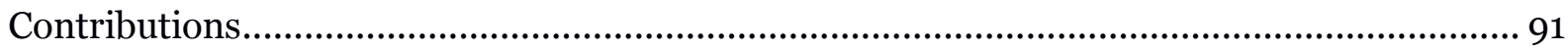

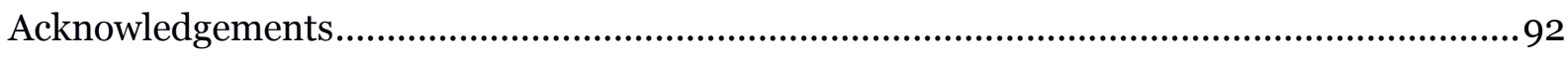

$\mathrm{CV}$ 


\section{Introduction}

The ability of the brain to rearrange its circuitry and adapt its functioning is termed neuroplasticity and underlies early brain development and complex brain functions, such as learning and memory formation. Furthermore, mechanisms responsible for neuroplasticity are of fundamental importance for recovery from brain injury and malfunctioning of such mechanisms plays a relevant role in the development of several neuropsychiatric diseases.

The cellular mechanisms associated with neuroplasticity have been a subject of extensive research and multiple methods have been developed to induce neuroplasticity in cell cultures and brain slices. Manipulation of human neuroplasticity in vivo can be achieved by invasive electric stimulation of the brain or with pharmacological interventions, which are often developed in an attempt to prevent, cure or delay the progression of diseases related to neuroplasticity alterations. However, pharmacological interventions affect the whole brain and are often accompanied by undesirable side-effects, consequently having limited application.

Non-invasive brain stimulation (NIBS) techniques have received increased attention in the last decades as methods for the research of plasticity in the intact human brain. Transcranial magnetic stimulation (TMS) is a well established technique while transcranial electric current stimulation (tES) methods have more recently gained popularity and demonstrated growing potential in basic neuroscience research as well as in numerous clinical applications.

This thesis addresses the effects of tES techniques on aspects of human healthy and pathologic cognitive functioning, using behavioural measurements and functional imaging methods. The first section presents the basic concepts concerning neuroplasticity and techniques of non-invasive brain stimulation, which are necessary for the understanding of the studies presented in the thesis. The second and the third sections describe the research projects that were performed, presenting and discussing the obtained results. Finally, the last section summarizes and discusses the overall findings and offers a perspective on future research directions. 


\subsection{Neuroplasticity}

The brain undergoes transformations throughout the lifespan in response to internal and external stimuli. This property is termed neuroplasticity. The changes in neural circuitry will ultimately result in an adjustment or modification of the exhibited behaviour (Ganguly and Poo, 2013; Lledo et al., 2006). One particular type of neuroplasticity is synaptic plasticity, which refers to the changes taking place at the synaptic level by an increase or decrease in strength or efficacy of the synapses. Such changes occur within a time span ranging from milliseconds to several minutes (short-term synaptic plasticity), or even lasting for hours, days and possibly longer (long-term synaptic plasticity) (Citri and Malenka, 2008). Several operating mechanisms have been described for each type of synaptic plasticity, two of which are long-term potentiation (LTP) and long-term depression (LTD) (Bliss and Cooke, 2011; Collingridge et al., 2010; Cooke and Bliss, 2006). Neuroplasticity is of outstanding relevance in several neural processes, and synaptic plasticity in particular is thought to be one of the mechanisms by which activity modulates the strength of existing synapses, playing an important role in establishing long lasting memory, knowledge and learning (Cooke and Bliss, 2006; Ganguly and Poo, 2013). Both LTP and LTD have mostly been studied in hippocampal slices, a structure known for its role in memory formation (Whitlock et al., 2006). Moreover, several studies have been able to manipulate performance of memory and learning tasks by inducing defective synaptic plasticity in pharmacological and animal studies (Lynch, 2004; Martin et al., 2000).

Macroscopic correlates of the neuroplasticity cellular mechanisms are evident as functional and structural brain reorganization, which can be observed using imaging methods such as magnetic resonance imaging (MRI) and positron emission tomography (PET) (May and Gaser, 2006; Pascual-Leone et al., 2011; Zatorre et al., 2012). For instance, functional reorganization has been observed in Braille readers, as an expansion of the cortical representation of the finger used for reading (Pascual-Leone and Torres, 1993). Examples of structural plasticity induced by experience can be found associated to the development of expertise by training (Ganguly and Poo, 2013). One widely studied example is that of changes in the brain of musicians (Fauvel et al., 2014; Herholz and Zatorre, 2012): musical training has been shown to produce structural changes in the auditory cortex (Bermudez and Zatorre, 2005) and motor systems (Hyde et al., 2009). A similar example in other field of expertise is that of mathematicians, who were found to have increased gray matter density in the parietal 
cortex (Aydin et al., 2007). However, even the simple action of studying for exams has been shown to increase gray matter thickness in the parietal cortex and the hippocampus (Draganski et al., 2006).

Another important role of neuroplasticity is rehabilitation after brain injury (Murphy and Corbett, 2009). One example is that of functional reorganization observed in recovery after stroke, such as an increase in the cortical representation of a muscle of an affected motor area after training. The extent of recovery is also related to plasticity between the injured and contralateral hemisphere (Ganguly and Poo, 2013).

In scenarios of neurodegeneration, such as Alzheimer's disease, Parkinson's disease and multiple sclerosis (MS), neuroplasticity often contributes to the delay on the manifestation of certain symptoms even when brain tissue degeneration is already occurring (Cramer et al., 2011). For instance, increased brain activation and functional connectivity alterations have been described in MS patients without cognitive deficits, which are thought to reflect compensatory mechanisms (Hulst et al., 2012; Mainero et al., 2004; Schoonheim et al., 2013).

However, several neuropsychiatric diseases are associated with diverse manifestations of maladaptive plasticity. Impaired plasticity caused by disruption of mechanisms similar to LTP and LTD during early development has been considered as one of the possible causes of autism (Ebert and Greenberg, 2013) and also impaired hippocampal synaptic plasticity has been related to schizophrenia symptoms such as hallucinations and cognitive impairment (Stephan et al., 2006). Other cases of maladaptive neuroplasticity are characterized by excessive plasticity, such as chronic pain (Saab, 2012) and focal dystonia (Lin and Hallett, 2009). Therefore, great effort is made not only to understand the underlying mechanisms, but also on the development of therapeutic strategies targeting modulation of neuroplasticity, by training and rehabilitation, pharmacological approaches, cell-based therapies or electrical stimulation techniques (Cramer et al., 2011; Ganguly and Poo, 2013). 


\subsection{Non-invasive brain stimulation}

Given the utmost relevance of neuroplasticity in both physiological and pathological aspects, the possibility of inducing and studying such processes in vivo in the human brain in a noninvasive manner, resorting to non-expensive tools presents enormous potential in basic research and therapeutics. Externally applied electric fields have been tested in animal and human studies, and it was observed that in brain slices both weak direct current (DC) fields and slow and fast oscillating fields are able to modulate neuronal excitability (Bikson et al., 2004). In humans, this can be achieved with NIBS techniques in a variety of ways.

One since long established technique is TMS, which consists on the application of magnetic pulses through the scalp. Such pulses induce a secondary electric field in the brain by electromagnetic induction. If strong enough, the magnetic pulse can even elicit neuronal action potentials without causing pain. Simple TMS applications include the monitoring of cortical excitability, for instance by evoking motor evoked potentials (MEPs) when it is applied over the motor cortex (Rothwell, 1993) or by eliciting phosphenes when applied over the visual cortex (Amassian et al., 1998). In addition, several TMS protocols, such as repetitive TMS at different frequencies, have been developed to modify neuroplasticity and for diverse therapeutic applications (Wassermann and Zimmermann, 2012).

Another type of NIBS technique is tES, which consists on application of weak electric currents through electrodes positioned on the scalp (Ruffini et al., 2013). In transcranial direct current stimulation (tDCS) the intensity of the applied current is constant through time, whereas in other methods such as transcranial alternating current stimulation (tACS) and transcranial random noise stimulation (tRNS), the current oscillates with a certain frequency (or range of frequencies), usually with a sinusoidal shape. These techniques are also able to induce changes in neuroplasticity in a non-invasive and painless manner and several studies have addressed the impact of tES on cognitive functions (for a review see Kuo and Nitsche, 2012), namely motor learning (Reis and Fritsch, 2011), working memory (Mulquiney et al., 2011; Teo et al., 2011), semantic and episodic memory (de Vries et al., 2009; Jacobson et al., 2012a; Manenti et al., 2013), categorization ability (Ambrus et al., 2011) and numeric skills (Kadosh et al., 2010; Snowball et al., 2013) among others, as well as its therapeutic applications in neuropsychiatric (Kuo et al., 2013) and neurologic diseases (Flöel, 2013). 


\subsubsection{Transcranial direct current stimulation}

The most widely used tES technique is tDCS, in which a weak current is applied through the scalp at a constant intensity, typically between $1-2 \mathrm{~mA}$. The earliest studies investigated the effect of tDCS on motor cortex excitability using TMS. This is convenient as the motor cortex is an output cortex: a TMS pulse is able to elicit action potentials on the targeted neurons or neuronal groups, which propagate along the corticospinal tract, eliciting movement. Typically, the cortical representation of small hand muscles in the primary motor cortex (M1) is targeted to elicit MEPs. The MEP amplitudes can therefore be compared before and after stimulation as a measure of change in corticospinal excitability of the motor system induced by tDCS. Using this method, the application of $4 \mathrm{~s}$ of anodal and cathodal tDCS was tested using several montages, and positioning one electrode over the M1 (often named "active" electrode) and the other (often named "reference" or "return" electrode) on the contralateral forehead (M1-ClF) proved the most effective. The stimulation induced an increase in excitability with the anode over the M1 and a decrease in excitability with the inverse polarity (Nitsche and Paulus, 2000), as reflected in the increase or decrease of MEP amplitudes, respectively. Subsequently, it was found that longer stimulation duration, particularly $13 \mathrm{~min}$ of anodal and 9 min of cathodal tDCS, was able to induce excitability changes for up to 30 minutes or even longer (Nitsche and Paulus, 2001; Nitsche et al., 2003a). These findings established the M1-ClF montage as the standard for M1 stimulation at the time, and prompted the exploration of a wider range of applications of tDCS as a tool for modulating neuroplasticity.

The effects elicited by tDCS depend on - but are not completely determined by - the following stimulation parameters: the current intensity, the duration of stimulation, the electrode size and the electrode positioning (referred to as the stimulation montage). It was initially observed that the strength and/or duration of the effects of tDCS on motor cortex excitability increased with higher current intensities, and that an increase in stimulation duration led to longer lasting effects (Nitsche and Paulus, 2001; Nitsche et al., 2003a). However, the relationship proved to be far more complex (Batsikadze et al., 2013; MonteSilva et al., 2013). For instance, doubling the intensity led to inverse effects after cathodal 
tDCS (Batsikadze et al., 2013) and increasing of the duration of anodal tDCS up to 26 min led to excitability decreases (Monte-Silva et al., 2013).

Both the electrode size and relative position are highly relevant factors. Typical electrode sizes range between $16 \mathrm{~cm}^{2}$ to $35 \mathrm{~cm}^{2}$. The stimulation montage was generally determined by placing the electrodes on top of the region of interest (see, for a compilation of montages used on tDCS studies Nitsche and Paulus, 2011; Nitsche et al., 2008). For example, in case of the M1, the location is often determined by identifying the cortical representation of the target muscle using TMS. Alternatively and for other brain areas, the positioning of electrodes can be made using the 10-20 EEG electrode system as a reference frame. However, even though the traditional montage $(\mathrm{M} 1-\mathrm{ClF})$ and electrode sizes were found to be optimal for immediate effects on modulation of M1 excitability (Nitsche and Paulus, 2000), it presents some limitations. First, even though approximately $90 \%$ of tDCS studies focus on the motor or visual cortex, it is important to note, specially for other applications, that the second electrode, usually placed over the contralateral forehead, is not functionally inert, as confirmed with, for instance neuroimaging studies (Polanía et al., 2011). Therefore, whatever the effect of this electrode on the prefrontal cortex, it should not be ignored. One proposed strategy to overcome the possibly undesired effect of the return electrode when aiming at an effect of stimulation under only one of the electrodes, consists on increasing the area of the return electrode while keeping the current constant, thus decreasing current density (current intensity/electrode area) and the effects under this electrode (Nitsche et al., 2007). Alternatively, researchers have placed the return electrode in an extracephalic position, such as the shoulder or the forearm (Cogiamanian et al., 2007; Moliadze et al., 2010).

A second limitation derives from the widespread electric field induced in the brain by such large electrodes and their relative positioning. The consequence is a relatively poor focusing which can make the interpretation of the results difficult when it is not possible to precisely pinpoint the structures affected by stimulation. In addition, it can prevent a more localized stimulation. However, it has been suggested that by reducing the electrode size it is possible to target a brain region with considerable precision (Nitsche et al., 2007). An alternative workaround for the focusing problem has been achieved by ingenious combinations of electrode size and montage, such as the one recently presented as high-definition tDCS (HDtDCS) (Minhas et al., 2010). This electrode montage consists on one small electrode (diameter $<12 \mathrm{~mm}$ ) positioned over the location of interest, surrounded by 4 equally small 
return electrodes in a ring-like configuration, and has been shown to modulate neuroplasticity of the M1 with greater spatial precision (Edwards et al., 2013; H.-I. Kuo et al., 2013).

A valuable contribution to the understanding of the problem of stimulation montage and electrode size has been given by computational studies modelling the electric field distribution induced by tDCS (for a review see Miranda et al., 2013). Although the earliest simulations used simplified spherical head models, these have evolved to highly detailed models based on individual structural MRI data, providing estimates of the electric field in the brain, which could not otherwise be easily obtained either in vitro or in vivo. Indeed, besides verifying the broad effect induced by typical stimulation electrodes, modelling studies observed that in a usual montage (two electrodes on the skull) the maximum of the electric field is not necessarily directly underneath the anode and cathode, but also lies between the two electrodes, thus questioning the established rationale for electrode positioning (Salvador et al., 2010).

In addition, the theoretical predictions suggest that factors, which cannot be determined by the experimenter, such as individual anatomical characteristics like skull morphology, play an important role with regard to the electric field induced in the brain (Datta et al., 2010). The manner in which the electric field is spatially distributed across the brain is of relevance, since studies in rat cortical neurons in vitro suggest that, besides cell type and morphology, the extent to which neurons are affected by tDCS depends as well on the orientation of the cells with regard to the induced electric field (Radman et al., 2009). Besides intrinsic stimulation parameters and anatomical features, the effect of stimulation is also strongly dependent on the functional state of the brain, before or during the application of the current, that is, whether the stimulation is given during rest or simultaneously with some motor or cognitive task (Antal et al., 2007).

To understand the relationship between all the relevant factors and the effects induced by tDCS, it is necessary to investigate the underlying mechanisms. It is thought that during tDCS, a shift occurs in the resting membrane potential of the neurons, in opposite directions according to polarity: anodal stimulation will shift the membrane potential towards depolarization whereas cathodal will more strongly hyperpolarize the neuronal membrane (Liebetanz et al., 2002; Nitsche and Paulus, 2000). Pharmacological studies carried out to further elucidate the mechanisms underlying the after-effects of tDCS suggest that these 
closely resemble LTP and LTD, as the induced long-lasting excitability changes were abolished by the administration of an NMDA receptor antagonist (Liebetanz et al., 2002; Nitsche et al., 2003a). In addition, it has also been suggested that in the development of the after-effects, both glial cells (Ruohonen and Karhu, 2012) and other non-synaptic mechanisms may play a role (Ardolino et al., 2005). In spite of the achieved progress, the mechanisms by which tDCS leads to long-lasting changes in the brain are still a matter of investigation.

\subsubsection{Transcranial random noise stimulation}

Another variant of transcranial electrical stimulation is tRNS. In this modality, the frequency of the applied alternating current is randomly sampled between 0.1 and $640 \mathrm{~Hz}$ and this frequency range is often divided into low-frequency (0.1-100 Hz, lf-tRNS) and highfrequency (101-640 Hz, hf-tRNS). The observed physiological effects following $10 \mathrm{~min}$ of tRNS at $1 \mathrm{~mA}$ peak-to-peak amplitude over the M1 were, similarly to anodal tDCS, an increase in cortical excitability lasting for 1 hour. When investigating separately the effects of high and low frequency ranges, it was found that the observed increase in M1 excitability was caused by hf-tRNS. Lf-tRNS had no effect on MEP amplitudes, as well as hf-tRNS applied over the premotor cortex (Terney et al., 2008). As previously described for tDCS (Antal et al., 2008b), the changes in excitability after tRNS are affected by the state of the brain during stimulation: contraction of the target muscle or attending to a cognitive task during stimulation leads to a decrease in M1 excitability. Offered explanations for the underlying mechanisms so far suggest that tRNS may reduce the time that sodium channels require to be able to repolarise after undergoing depolarization, thus enhancing their activity (Terney et al., 2008). To explain the differential effects of low and hf-tRNS and anodal tDCS, is was suggested that a summation of sub-threshold stimuli could be taking place, when the frequency would be high-enough to present two stimuli within the period of the time constant of a neuron (Fertonani et al., 2011). Experimentally, tRNS has the advantage of allowing better blinding of the participants than tDCS, as it causes reduced cutaneous perception (Ambrus et al., 2010). However, when considering the possibility of replacing excitatory tDCS with this technique, one needs to take into account the differences in mechanisms of action. 


\subsubsection{TES and motor learning}

With the effects of tDCS having initially been studied most extensively in the motor cortex, a bulk of the research on tDCS has been dedicated to several aspects of motor function and learning. One early study showed that implicit motor learning could be improved by applying anodal tDCS over M1, during a serial reaction time task (SRTT) (Nitsche et al., 2003b). This was interpreted as a beneficial effect of plasticity increase during learning and boosted research of tDCS as an enhancer of motor function, motor learning or cognitive functioning in general.

The timing of stimulation with regard to the task or training (stimulation before learning or during learning), seems to be critical for the obtained results. Indeed, a later study using again the SRTT and the same stimulation parameters reported no changes in performance when anodal tDCS was applied before the task, instead of simultaneously (Kuo et al., 2008). The differential effect of stimulation according to polarity is unclear, as several studies use only anodal tDCS, a few observe no effects after cathodal stimulation, whereas in others polarity interacts with the timing of stimulation (Jacobson et al., 2012b; Reis and Fritsch, 2011). For instance, on tasks involving motor and visual integration, learning was improved by anodal tDCS over M1 or visual area $\mathrm{V}_{5}-\mathrm{Cz}$ whereas cathodal tDCS had no effect (Antal et al., 2004a); however, once learning had been stabilized, cathodal tDCS further improved performance, while anodal tDCS had no effect (Antal et al., 2004b). It was suggested that increasing plasticity with anodal tDCS was beneficial during learning whereas in later stage, cathodal stimulation had the advantage of reducing signal to noise ratio in the brain, contributing to improved performance. Further interactions between the timing of stimulation and polarity were found in the study by Stagg and colleagues (2011) where the rate of learning of an explicit motor sequence task was faster during anodal tDCS and slower during cathodal tDCS over the M1, but learning was slowed regardless of polarity, when the task was performed after stimulation. In addition, carefully choosing the timing of stimulation in combination with training can possibly lead to longer-lasting effects: the improvement achieved with anodal tDCS over the M1 during the performance of a sequential visual isometric pinch task (offline effect) for 5 consecutive days was still present 3 months later, in comparison with sham stimulation (Reis et al., 2009).

Another important factor is the intensity of the current applied during tDCS. The complex relationship between the effects induced by tDCS and the intensity with which it is applied 
has not often been addressed in cognitive studies. Cuypers and colleagues (2013) compared the effect of anodal tDCS (20 min) over the M1 with $1 \mathrm{~mA}$ or $1.5 \mathrm{~mA}$ intensity in an explicit motor sequence learning task. They observed that both intensities improved performance, but only higher intensity resulted in significant effect.

Although the focusing of most tDCS methods is poor, montage and region of stimulation are relevant, and by comparing results of different stimulation locations one can infer the differential role of specific brain areas. Contribution to the clarification of the specific roles of the M1 and the cerebellum to the adaptation of visuomotor transformations came from a study where using anodal tDCS (15 min, $2 \mathrm{~mA}$ ) on either of the regions it was found that the cerebellum was involved in the adaptation to the transformation whereas the M1 influenced retention of the adaptation (Galea et al., 2011). Also lateralization aspects of motor skill learning have been addressed using tDCS, by testing hemispheric specialization. It was found that anodal tDCS of the left M1 (shoulder cathode, $1 \mathrm{~mA}, 20 \mathrm{~min}$ ) led to greater skill learning with either hand, which was not found when stimulating the right M1 (Schambra et al., 2011). The authors interpreted this result as evidence of specialization of the left M1 for motor skill learning, which had already been found for representation of learned actions. This further suggests that effects of tDCS on general motor function can also depend on whether the stimulated hemisphere is dominant or non-dominant. Results of this study also support the idea that bihemispheric stimulation of the motor cortex is more effective on motor skill improvement that unihemispheric. Also, Boggio and colleagues (2006) compared motor function in a small set of healthy subjects after anodal tDCS (M1-ClF, $20 \mathrm{~min}, 1 \mathrm{~mA}$ ) and found improvements only when the non-dominant hemisphere was stimulated. Results from a subsequent study suggest that this effect can be enhanced, when simultaneous cathodal stimulation of the dominant M1 is applied (Vines et al., 2008) showing that tDCS can be used to modulate inter-hemispheric functional relations.

Another aspect of motor learning is the ability to generalize and transfer previously learned movements (Krakauer and Mazzoni, 2011). To study the relationship between this process and motor cortex plasticity, tDCS (1 mA, $20 \mathrm{~min}$ ) was applied during a force field adaptation task (Orban de Xivry et al., 2011). In this study, it was reported that the application of tDCS over the M1 increased generalization in intrinsic coordinates, regardless of the polarity, contributing to the evidence that this phenomena depends on plasticity in the sensorimotor cortex. 
Further efforts to understand how tDCS affects different learning stages were made by Saucedo Marquez and colleagues (2013). They compared online, offline and retention effects of anodal tDCS (1 mA intensity, 20 min duration, cathode over the shoulder) of the right motor cortex for an explicit sequence tapping task and for a sequential visual isometric pinch task performed with the left hand. Although anodal tDCS improved learning for both tasks, this happened in different stages for each task. The sequential task benefited from stimulation during online learning, whereas for the force task the stimulation acted mainly on retention, which can be explained in part by the contributions of the M1 for a different stage on each task.

There is considerably less research combining tRNS with cognitive paradigms. As an excitability-enhancing method, the effect of tRNS on implicit motor learning was comparable to that of anodal tDCS, causing significant improvement when applied over the M1 during a SRTT (Terney et al., 2008). Similarly, when applied over the right dorsolateral prefrontal cortex (DLPFC) (return electrode over Cz), tRNS (full range) impaired categorization learning in a comparable way to anodal tDCS (Ambrus et al., 2011). However, contrarily to anodal tDCS, 10 min of hf-tRNS over the left DLPFC failed to induce detectable changes on the performance of a 2-back task (Mulquiney et al., 2011). Another differential effect of these two types of stimulation was described by Fertonani and colleagues (2011), who found that only hf-tRNS (at $1.5 \mathrm{~mA}$ ) over the visual cortex improved orientation discrimination when applied during a perceptual learning task. However, in a subsequent study, anodal tDCS led to significantly improved performance, when applied before the same task, instead of simultaneously, thus suggesting that the difference in the underlying mechanisms of the two types of stimulation is likely to dictate differences in the stimulation protocols, namely the timing (Pirulli et al., 2013). As described for tDCS, timing is a relevant factor and coupling of hf-tRNS with a deeply encoding cognitive training paradigm can lead to a long-lasting effect of the stimulation (Cappelletti et al., 2013; Snowball et al., 2013). In a recent study, hf-tRNS was applied for 5 consecutive days over the DLPFC bilaterally while subjects practised arithmetic exercises for 22 minutes, causing an improvement that was still detectable after 6 months (Snowball et al., 2013) whereas bilateral parietal stimulation increased numerosity discrimination ability (Cappelletti et al., 2013). There are no systematic studies testing the influence of different stimulation intensities. However, the skin perception, when using 
alternating currents is reduced, offering the possibility of applying higher intensities of stimulation without loosing the ability to sham.

Therefore, in spite of the many unanswered questions regarding the underlying mechanisms and ideal choice of parameters for the best stimulation protocols for enhancement of performance, tDCS studies have contributed to understanding certain aspects of motor learning.

\subsubsection{Clinical applications of tDCS}

Transcranial stimulation techniques have been widely tested as therapeutic agents in several neurological (Flöel, 2013) and psychiatric disorders (M.-F. Kuo et al., 2013). One major field of application of tDCS is in motor rehabilitation after stroke. It is generally accepted that the rehabilitation potential after stroke is associated to the degree of imbalance between the lesioned and contralateral hemispheres: the spared hemisphere shows increased activation, which is higher in patients who are less able to recover (Stagg and Johansen-Berg, 2013). Thus, tDCS has been used with the aim to reduce this imbalance, either by applying anodal tDCS over the M1 of the lesioned hemisphere or by applying cathodal tDCS over the contralesional M1, in both cases with an M1-ClF montage, or even, by using bilateral stimulation with the anode over the lesioned M1 and the cathode over the contralateral M1 simultaneously (Lindenberg et al., 2010). All in all, the use of tDCS after stroke, which is also possible in combination with other rehabilitation strategies, has shown promising results (Nair et al., 2011).

In the treatment of depression, tDCS approaches (Nitsche et al., 2009) aim at targeting the abnormal activity and plasticity of the prefrontal cortex, particularly the decreased activity of the left hemisphere. Typical protocols apply anodal tDCS over the left DLPFC at with an intensity of $2 \mathrm{~mA}$ with a diversity of montages, with the return electrode over the contralateral forehead (Boggio et al., 2008a), in an extra-cephalic position (Martin et al., 2011), or even bilateral stimulation (Brunoni et al., 2011), usually for several consecutive days. The results are promising with the effects of tDCS being able to reach those of pharmacological interventions, and the two approaches combined can be even more effective (Brunoni et al., 2013).

TDCS has also been helpful in the management of pain of diversified origin, applied either over the M1 or DLPFC to target regions belonging to the pain processing network or involved 
in pain-related affections, respectively. When applied over the M1, anodal tDCS has, for instance, reduced pain after spinal cord injury (Felipe Fregni et al., 2006a) or in fibromyalgia (Felipe Fregni et al., 2006c; Riberto et al., 2011; Valle et al., 2009), and 5 consecutive days of stimulation improved chronic neuropathic pain in patients with MS. Anodal tDCS over $\mathrm{C}_{3}$ or $\mathrm{C}_{4}$ (ref contralateral) was applied for 5 consecutive days at $2 \mathrm{~mA}$ (parallel groups). The effect was significant and $60 \%$ of patients reported reduced pain by 50\% or more after 4 weeks (Mori et al., 2010). The same protocol improved tactile perception also in MS patients (Mori et al., 2013) and reduced pain of diverse origins for several weeks in chronic pain patients (Antal et al., 2010). Also concerning migraine has tDCS shown therapeutic potential. The most successful approach so far, consists on applying cathodal stimulation over the occipital cortex with the goal of reducing the hyperexcitability of the visual cortex that is characteristic of migraine patients (Antal et al., 2005; Chadaide et al., 2007, Antal et al., 2011).

Other explored applications include tinnitus, in which anodal tDCS of the left temporoparietal area is able to reduce the intensity (Fregni et al., 2006; Shekhawat et al., 2013) whereas over the prefrontal cortex can be used to reduce its perception (Faber et al., 2012); bilateral DLPFC stimulation against alcohol or cigarette addiction (Boggio et al., 2008b; Fregni et al., 2008); cathodal tDCS of the left temporoparietal cortex (TPC) for reduction of auditory hallucinations in schizophrenia (Brunelin et al., 2012), cathodal tDCS for excitability reduction in epilepsy (Nitsche and Paulus, 2009), as well as several stimulation protocols for improving cognitive performance in, for instance, mild cognitive impairment and Alzheimer's disease dementia (Boggio et al., 2012, 2009; Ferrucci et al., 2008). In spite of the promising results, the progress in therapeutic applications of tDCS has been slowed by the incomplete understanding of the mechanisms by which tES techniques, and tDCS in particular, act in the brain.

\subsubsection{Combining tES with fMRI}

TMS derived measures provide a relatively direct assessment of tDCS-induced changes in excitability, but their use is inherently limited to measures of the stimulation effect on the motor cortex, whether it is caused by direct stimulation or indirectly by acting on other areas to which it is connected. Once established the neuromodulating potential of tDCS, a wide range of possible applications presents itself, together with the need to better understand the effects in other brain regions. One of the techniques that can be used to gather whole-brain 
data is functional MRI (fMRI). Although measuring brain activity indirectly, it allows for a simultaneous investigation of events in any brain region with high spatial resolution. What is more, the diversity of analytical approaches makes this technique a versatile manner to explore numerous aspects related to the stimulation (Saiote et al., 2013b).

When combining fMRI with tES, certain technical challenges have to be taken into account. Regarding the safety of the procedure, the main concern is that the radio-frequency pulses of the scanner can cause heating under the electrodes (Lemieux et al., 1997). The solution to this problem consists on installing resistors on the electrodes' wires close to the electrodes. Other technical difficulties concern the quality of the acquired data. Even when stimulation and image acquisition do not occur simultaneously, it is important to verify if the stimulation equipment causes a detrimental effect on image quality. In previous studies, it has been shown that only a small reduction (between 3 and 8\%) in signal-to-noise ratio (SNR) (Antal et al., 2011) and no distortion were found in the structural or functional images, as long as the electrode cables were unplugged from the stimulator (Polanía et al., 2011), and even when the images are acquired while the stimulation is applied, the changes in SNR remain minimal (Antal et al., 2011). However, the stimulation can cause, for instance, mild susceptibility artifacts under the electrodes (Antal et al., 2011) as well as Bo field distortions (Holland et al., 2011), which have been limited to the scalp and did not reach the brain tissue in the cases reported so far. Accordingly, in a recent study using 2 post-mortem subjects, the highest artifacts induced by tDCS on functional images were found in the scalp and in the cerebrospinal fluid (CSF) at the surface and in the ventricles (Antal et al., 2014). Nevertheless, the effect induced by tDCS was found to be of comparable magnitude (approximately half) to that of the response elicited during a finger-tapping task, using the same imaging sequence. Therefore, studies with joint application of tDCS and fMRI, specially when applied concurrently, should include a control for artifacts and take it into consideration in the interpretation of results.

In the simplest adaptation of basic TMS studies, fMRI activation elicited by simple motor tasks confirmed that a variety of tDCS protocols over the M1 is able to modulate diverse motor areas differentially (Antal et al., 2011; Baudewig et al., 2001; Kim et al., 2012). In agreement, after 10 min of anodal stimulation over the M1, whole brain analysis of activation during an implicit motor learning task showed increased activity in the left M1, left dorsal premotor cortex (PMd), and bilateral supplementary motor area (SMA) (Stagg et al., 2009). 
As found in several studies of motor learning discussed above, the inverse effect of cathodal tDCS on cortical excitability did not translate to motor related activation, as bilateral M1, PMd, and posterior parietal cortex (PPC) showed increased activity compared to sham. Also, a region of interest (ROI) analysis in the same study, found that anodal tDCS was related to an increase in activation in the left M1 comparing to sham, whereas cathodal tDCS was associated with increased activation in the contralateral M1 and PMd. Another important result of this study provides further support for task specificity of the effects of tDCS: regardless of the polarity, neither a ROI on the frontopolar cortex under the reference electrode, nor a ROI at the primary visual cortex (V1) chosen as control, showed a stimulation effect.

Besides observing how tDCS affects activity of brain regions in an isolated manner, it is of added interest to investigate how it modulates the functional interaction of brain regions with one another, that is, their functional connectivity. Such analysis techniques also make it possible to study spontaneous brain activity in the absence of a task or external stimuli. Several resting-state fMRI studies have shown spontaneous coherent brain activity happening at low-frequencies $(0.01-0.1 \mathrm{~Hz})$, defining sets of distinct brain regions (networks) which are functionally relevant, and comply with the underlying anatomy (Biswal et al., 1995; De Luca et al., 2006). Currently there are several methodological approaches to such data. The simplest is to calculate the correlation between signal from different areas (either at voxel resolution or by grouping areas of interest). To further interpret the results, one possible approach to characterize local and global properties of functional (or structural) networks is derived from graph theory (Bullmore and Sporns, 2009; Stam and Reijneveld, 2007). In this framework, a network is formally defined as a graph (a set of nodes and edges that connect them). The most basic measure is the connectivity degree (K), which quantifies the number of connections of a node. The characteristic path length (L) can provide information about the global character of connections, as it quantifies the minimum number of connections between two nodes, thus measuring whether they are directly or indirectly connected. Several studies using graph theory have been able to detect abnormalities in structural and functional networks in the context of a variety of neurological and psychiatric disorders (e.g. Schoonheim et al., 2013; Stam et al., 2007). Independent component analysis (ICA) is another approach, where the data is decomposed into spatially independent maps 
and the associated timecourses, commonly used to identify resting-state networks, such as the default mode network (DMN) (Beckmann et al., 2005; Calhoun et al., 2001).

In the first study of the effects of tDCS on resting-state brain activity, Polanía et al. (2011) found that $10 \mathrm{~min}$ of anodal tDCS at $1 \mathrm{~mA}$ (left M1-CSR) reduced the direct connections of the left primary somatosensory cortex (SM1) to distant brain regions, increasing its connectivity with the left premotor and M1 as well as with the left SM1 and superior parietal cortex. Furthermore, the stimulation induced changes in the connections within the DMN and executive control network, both usually identified in resting state studies.

Besides cortical effects, tDCS was found to alter cortico-subcortical resting-state functional connectivity (Polanía et al., 2012a). Taking the nucleus accumbens, caudate, putamen, and thalamus as seeds in a multiple regression analysis, it was found that after $10 \mathrm{~min}$ of anodal tDCS over the M1, functional connectivity increased between the left thalamus and M1 as well as between the left caudate and superior parietal lobule, and decreased between the left caudate and the posterior cingulate cortex (PCC). Reversal of polarity induced a connectivity decrease between the right putamen and left M1 and between the right thalamus and left superior frontal gyrus. Recently, cathodal tDCS (10min, 1mA, M1-ClF) was found to induce increased functional connectivity within the motor network and the DMN using ICA, whereas anodal stimulation had no effect. Furthermore, using a ROI approach, increased connectivity between left and right M1 and between left and right SMA were found (Amadi et al., 2013)

In further support of functional specificity of the effects induced by tDCS, changes within the DMN were found in two other resting state studies stimulating the DLPFC (Keeser et al., 2011; Peña-Gómez et al., 2012) with $2 \mathrm{~mA}$ for $20 \mathrm{~min}$. The first study reported changes in frontal areas of the DMN and the second a loss of synchronization between the frontal and posterior regions of the DMN. Connectivity was also altered in the left and right attention networks (Keeser et al., 2011), which could reflect a modulation of the level of alertness, as well as the anti-correlated network (Peña-Gómez et al., 2012). Interestingly, the second study found no changes the motor or visual networks.

The number of studies combining fMRI with tRNS is very limited. A ROI analysis of the left sensorimotor cortex showed that after $4 \mathrm{~min}$ of stimulation $\left(\mathrm{C}_{3}-\mathrm{ClF}\right)$ at $1 \mathrm{~mA}$ the extent of activation elicited by a finger-tapping task was decreased (Chaieb et al., 2009) but there are no studies about the effects of tRNS on distant brain regions or on functional connectivity. 


\subsection{Multiple Sclerosis}

Multiple sclerosis (MS) is a chronic inflammatory disease of the central nervous system (CNS) mainly characterized by the destruction of the neuronal myelin sheath. The estimated prevalence of MS in Europe is 83 per 100000, with rates being approximately twice as high for women as for men, and lower in the southern than in the northern European countries (Pugliatti et al., 2006).

\subsubsection{Symptoms and disease progression}

The lesions caused by demyelination can occur anywhere in the brain as well as in the spinal cord, resulting in a wide range of symptoms that can be experienced by the patients. Disturbances in the motor system are among the most common, followed by the visual, urinary and sensory domains. Other common symptoms include cognitive impairment, and neuropsychiatric disturbances. In addition to demyelination, axonal damage and loss is known to occur and greatly contribute to disability.

The progression of the disease can follow 4 distinct courses: relapsing-remitting (RRMS), primary progressive (PPMS), secondary progressive (SPMS) and progressive relapsing MS (PRMS). RRMS is the most common (80\%) initial disease course and is characterized by isolated events of acute neurological symptoms (known as relapses), which are followed by complete remission and separated by periods with no disease progression. In PPMS there is a constant gradual worsening of symptoms without acute attacks. After some years, $65 \%$ of patients with RRMS often shift to such a gradual progression of the disease, a course which is then designated as SPMS. When the disease manifests with a gradual worsening of symptoms in addition to acute relapses, it is named PRMS, occurring less frequently.

The origin of MS is not fully explained, likely resulting from a combination of genetic and environmental factors. Current treatment strategies aim at slowing the progression of the disease using immunosuppressive therapy to reduce the frequency of relapses, and also involve corticosteroids to treat relapses and eventually adjuvant therapy for managing specific symptoms. 


\subsubsection{Contribution of MRI}

Conventional MR imaging is used to assist in diagnosis of definite MS (Polman et al., 2011), by allowing identification of lesions disseminated in space and time, as well as other aspects of brain injury (Filippi et al., 2012). Lesions can be visible as hyperintensities in T2-weighted images. In addition, new inflammatory lesions are characterized by a breakdown of the blood-brain barrier and can be made visible by injecting the contrast agent gadolinium, during T1-weighted MRI. Often (40\%), new lesions become chronic lesions visible in T1weighted images as hypo-intensities ("black holes"), corresponding to more severe tissue damage including both demyelination and axonal loss. However, conventional MR imaging of MS lesions presents as limitation the lack of specificity regarding the underlying pathological events, not allowing a distinction between inflammation, de- and remyelination, edema, gliosis and axonal loss (Filippi and Rocca, 2011). As a result, lesion load does not necessarily strongly correlate with clinical presentation and disability, which if of referred to as the clinico-radiological paradox (Barkhof, 2002), thus limiting the usefulness of conventional MRI in prognosis of the disease after diagnosis.

Other MR methods and imaging techniques are used to overcome this paradox. Gray matter (GM) damage seems to better correlate with progression of disability and cognitive impairment (Filippi et al., 2010). Lesions in GM are very hard to identify by using conventional MRI methods due to their small size and poor contrast to normal GM. However, cortical lesions can more easily be identified by using double-inversion-recovery MRI sequences instead of fluid-attenuated inversion-recovery (FLAIR) or T2-weighted imaging, or by imaging at higher field strengths (Filippi et al., 2014; Wattjes et al., 2007).

MRI can also be used to measure cortical atrophy, which progresses at a rate of $0.7-1 \%$ decrease in brain volume per year, in MS patients with diversified courses (Miller et al., 2002). As observed with GM lesions, GM atrophy in particular is associated with increased disability and cognitive impairment, and starts occurring early in the course of the disease, although different structures seem to be affected at different stages of the disease, as revealed by studies of voxel-based morphometry. Measurement of GM atrophy of specific structures can be helpful to assess specific domains of disability and symptoms, such as memory or fatigue (Riccitelli et al., 2011b; Yaldizli et al., 2013, 2011).

Changes in diffusivity found with Diffusion-Tensor imaging (DTI) can be used to track tissue damage, as they reflect axonal loss and demyelination (Roosendaal et al., 2009). For 
instance, in T2 lesions there is an increase in mean diffusivity (even before lesion formation) and a decrease in fractional anisotropy (FA) (Filippi and Rocca, 2011). Also, changes in FA have been associated with cognitive impairment in MS (Bester et al., 2013; Genova et al., 2013). Also magnetization transfer MRI can serve as measure of tissue structure destruction even before the formation of a contrast enhancing lesion, and can increase when remyelination occurs. Magnetization transfer of GM and normal appearing white matter (WM) has been shown to correlate with disability and cognitive impairment better that T2 lesion load alone (Filippi and Rocca, 2011). Other MR based techniques that contribute to MS research are MR spectroscopy, which can provide valuable information about alterations in cell metabolism, and also perfusion weighted MRI and imaging of iron deposition (Filippi and Rocca, 2011).

\subsubsection{Fatigue in Multiple Sclerosis}

Among the most common symptoms affecting MS patients is fatigue, with a reported prevalence of up to $75 \%$ (Lerdal et al., 2007). Fatigue is many times considered by the patients as their most disabling symptom and has a profoundly negative impact on quality of life (Janardhan and Bakshi, 2002). Fatigue in MS has been described in a variety of ways such as lack of energy (Comi et al., 2001; Giovannoni, 2006), pathological exhaustion, an overwhelming sense of tiredness distinguishable from sadness or weakness, difficulty initiating or sustaining voluntary effort (Chaudhuri and Behan, 2004; Comi et al., 2001; Giovannoni, 2006; Krupp et al., 1988), or, in an attempt to combine physical and mental symptoms, as a "subjective lack of physical and/or mental energy that is perceived by the individual or caregiver to interfere with usual and desired activities" (MS Council, 1998), often not subsiding after bed rest.

Reflecting the complexity of fatigue in MS, numerous questionnaires have been devised to quantify subjective self-reported fatigue. The available questionnaires use different approaches in the assessment of fatigue, either trying to directly quantify it, or rather focusing on the impact it has on daily life, putting emphasis primarily on the psychological, cognitive or physical aspects of fatigue, and have different degrees of specificity towards MS (Amtmann et al., 2012). Among the most common fatigue scales are the Fatigue Severity Scale (FSS) (Krupp et al., 1989), the MS-specific Fatigue Severity Scale (MSFSS) (Krupp et al., 1995), the Modified Fatigue Impact Scale (MFIS) (MS Council, 1998) and the Chalder 
fatigue scale (Chalder et al., 1993). In addition, fatigue is often quantified with the aid of a visual analogue scale (VAS) of, typically, $10 \mathrm{~cm}$.

In spite of the considerable attention given to this symptom, the causes and underlying mechanisms leading to fatigue remain, to a great extent, unknown. Regarding its pathophysiology, one proposed intervening factor in fatigue are certain cytokines acting as inflammatory mediators in MS. Evidence was found of elevated production of Interferon- $\gamma$ (IFN- $\gamma$ ), tumour necrosis factor- $\alpha$ (TNF- $\alpha$ ) and Interleukin-6 (IL-6) in MS patients with fatigue (Heesen et al., 2006; Induruwa et al., 2012). It is also possible that endocrinal dysfunction and MS fatigue are related, as it happens with other autoimmune diseases where fatigue manifests, such as in chronic fatigue syndrome and lupus. Fatigue severity was associated with dysregulation of the hypothalamo-pituitary-adrenal (HPA) axis, found to have higher reactivity in patients with fatigue but other studies provide conflicting results (Braley and Chervin, 2010; Induruwa et al., 2012). In addition, one study found an association between damage to the hypothalamus and fatigue, as fatigue severity correlated with T1 relaxation times within the hypothalamus (Zellini et al., 2009). Although one part of this thesis focuses mainly on primary fatigue, it is worth to note that fatigue symptoms can be secondary to other conditions present in MS, such as depression, sleep disorders (sleep apnea, insomnia or restless leg syndrome) or disability, which can also contribute to the severity of primary fatigue symptoms (Braley and Chervin, 2010).

The contributions of MRI techniques to understand the mechanisms of fatigue in MS patients have been somewhat inconsistent and discrepant results regarding atrophy and lesion load measures have successively been presented. Concerning lesion load, although a correlation of global lesion load and fatigue severity has been reported in a few studies (Colombo et al., 2000; Sepulcre et al., 2009), most have been unable to find a significant association of fatigue with lesion load (Riccitelli et al., 2011a; Tartaglia et al., 2004; Tedeschi et al., 2007; van der Werf et al., 1998; Yaldizli et al., 2011) or even with frequency of enhancing lesions (Mainero et al., 1999). However, lesion load does not reflect the complete extent of axonal damage, which can happen diffusely throughout the brain and can be associated with fatigue. Indeed, in a study using proton magnetic resonance, the NAA:Creatinine (NAA/Cr) ratio was significantly higher in the group of patients with stronger fatigue symptoms, indicating higher axonal damage and the FSS scores correlated inversely with NAA/Cr ratio (Tartaglia et al., 2004). 
Although a few studies were equally unable to report a correlation of fatigue with atrophy measures (Bakshi et al., 1999; van der Werf et al., 1998), recently it has been possible to relate fatigue to abnormalities in specific brain structures. For instance, Riccitelli et al. (2011) found correlation between FSS scores and GM atrophy in the central sulcus and precentral gyrus and a correlation of MFIS fatigue severity with GM atrophy of the left superior frontal gyrus and bilateral middle frontal gyri had been described by Sepulcre et al. (2009). Also, severity of fatigue symptoms was associated with progression of atrophy of the corpus callosum over 5 years in MS patients (Yaldizli et al., 2011). Another study comparing MS patients with or without fatigue, found that fatigue correlated with cortical atrophy of the parietal lobe (Pellicano et al., 2010). Deep white matter in the left frontal lobe was also implicated in fatigue, as changes in DTI correlated with MFIS scores (Pardini et al., 2010). It is possible that different aspects (motor or cognitive/mental) of fatigue are associated with damage to distinct brain regions.

Recently, fatigue has been associated with damage specific to the anterior thalamic tracts (Bester et al., 2013). Other previous studies have implicated abnormalities in the deep gray matter in MS fatigue symptoms. For instance, increased fatigue severity has been correlated to decreased cerebral blood flow and cerebral blood volume in the thalamus, putamen and caudate (Inglese et al., 2007) and patients with fatigue displayed reduced glucose metabolism in the basal ganglia, in comparison with patients without fatigue (Roelcke et al., 1997). Also, T1 relaxation times of the thalamus correlated with FSS scores (Niepel et al., 2006) and $\mathrm{NAA} / \mathrm{Cr}$ in the basal ganglia was decreased in fatigued MS patients (Téllez et al., 2008), both indicating that damage to these structures which can go unnoticed in macroscopic MRI can contribute to fatigue symptoms.

Besides the evidence of frontal and parietal reduction in glucose metabolism in MS patients with fatigue provided by an early PET study (Roelcke et al., 1997), further functional brain alterations associated with fatigue have been found using fMRI. For instance, while performing a simple motor task, fatigued MS patients had reduced activation in the contralateral middle frontal gyrus and thalamus, and increased activation of the contralateral cingulate motor area (Filippi et al., 2002). Also, cognitive fatigue was associated with abnormal activation in distinct frontal, parietal and occipital regions, as well as thalamus and basal ganglia (DeLuca et al., 2008). Recently, fatigue perceived by MS patients was associated with increased activation in the left posterior parietal cortex and the right 
substantia nigra (Engström et al., 2013) and in the caudate (Genova et al., 2013). Nevertheless, functional MRI remains a less explored technique for studying fatigue in MS.

Putting together the insufficient understanding of the mechanisms involved in fatigue, and its subjective nature and complexity, as well as the diversity of measuring scales, it is not surprising that the current therapeutic success is limited. Indeed, pharmacological agents have not been sufficiently successful in treating fatigue. The use of amantadine against fatigue symptoms has been the subject of considerable research, but overall presents mild positive results at best (Braley and Chervin, 2010; Krupp et al., 1995). The same can be said of modafinil, used in the context of several sleep disorders, often used by clinicians to manage fatigue symptoms, but with unconvincing results in controlled trials in MS (Lange et al., 2009; Rammohan et al., 2002; Stankoff et al., 2005). Other alternative approaches are available to manage fatigue symptoms, such as cognitive behavioural therapy (van Kessel et al., 2008), aerobic exercise and rehabilitation (Brown and Kraft, 2005), cooling therapy and energy conservation strategies (Schwid et al., 2003), often used in combination with pharmacological treatment (Braley and Chervin, 2010). Therefore, despite the range of possible strategies, considerable difficulties remain in the successful management of fatigue in MS. 


\subsection{Aims}

The aim of this work was to investigate the potential of tES techniques in modulating behaviour - known to depend on neuroplasticity - using fMRI to track the changes induced by stimulation on the whole brain.

Project 1 aims at addressing the application of tES techniques in a healthy population, evaluating its impact on motor learning guided by visual feedback. This project builds on previous research concerning visuomotor learning and tES by comparing several modalities of tES within one study, and tracking changes in brain activation occurring simultaneously with stimulation.

Project 2 focuses on therapeutic applications of tES by assessing the ability of tDCS to modulate self-reported fatigue in patients suffering from MS, testing its potential as an alternative strategy for management of this symptom as well as evaluating the existing expectations concerning anatomical substrates of fatigue.

In combination, the two studies aim to gather new knowledge concerning the global effects of the stimulation, the underlying mechanisms as well as the practical factors that should be taken into account, relevant for the future of diverse applications of tES techniques. 


\section{Impact of tDCS and tRNS on visuomotor learning behaviour and associated brain activity}

The aim of the first project was to investigate, how the dynamics of visuomotor learning are modified by external modulation of excitability of the motor cortex. The acquisition of a new motor skill is characterized by an initial learning stage, where considerable improvement of skill performance occurs within a short period of time, followed by a second stage of slower progress in learning. Specific patterns of brain activity, which have been identified in many fMRI studies correspond to each learning stage (Dayan and Cohen, 2011; Floyer-Lea and Matthews, 2005, 2004; Tomassini et al., 2011). For instance, activation of the M1, SMA, caudate and other posterior parietal and frontal regions decreases during early learning, whereas the activation of the putamen, thalamus and cerebellar dentate shows the opposite behavioural pattern (Floyer-Lea and Matthews, 2004). Long term motor learning, however, has been associated with increased activation of regions in the sensorimotor cortex and striatum (Floyer-Lea and Matthews, 2005). As mentioned previously, tES techniques can modulate motor learning in a timing-dependent manner (for a review, see Reis and Fritsch, 2011).

In this study, anodal tDCS, cathodal tDCS, hf-tRNS (101-640 Hz), lf-tRNS (0.1-100 Hz) or sham stimulation was applied during the initial learning stage. The visuomotor tracking task was adapted from previous studies and requires learning a pattern of variable hand pressure movements according to specific visual feedback (Floyer-Lea and Matthews, 2004). Besides behavioural performance, the effects of tRNS and tDCS during the task on functional networks were studied using fMRI.

According to the results previously described in the literature, it was expected that anodal tDCS would induce an improvement in performance during the first stage of learning. Considering that the effects of hf-tRNS are to some extent comparable to those of anodal tDCS, a similar or even greater increase in performance due to such stimulation was expected (Terney et al., 2008). In comparison with hf-tRNS, it was hypothesised that lf-tRNS would be responsible for a smaller, if any, improvement of performance, in parallel with the results presented in studies of brain excitability (Fertonani et al., 2011; Terney et al., 2008). Furthermore, we were interested in observing whether possible stimulation-induced 
differences between groups would be observable and maintained after stabilization of performance.

The results have been published in PlosOne under the title: "High-Frequency TRNS Reduces BOLD Activity during Visuomotor Learning“ (Saiote et al., 2013a). 


\subsection{Methods}

\subsubsection{Subjects}

In total, 52 healthy participants took part in the study (22 male, mean age: 27.66 years, age range: 20-50 years). Two participants were excluded for not being able to properly understand the task. Thus, data from 50 subjects was analysed, equally divided into 10 subjects per stimulation condition: anodal tDCS ( 3 male, mean age: 28.68 years, age range: 22-50 years), cathodal tDCS (6 male, mean age: 25.64, age range: 20-32 years), highfrequency tRNS (4 male, mean age: 27.63, age range: 20-27 years), low-frequency tRNS (7 male, mean age: 31.67 , age range: 24-37 years) and sham (2 male, mean age: 28.67 , age range: 23-44 years). The participants fulfilled the following criteria: no history of neurological or psychiatric disorders, no drug abuse, no alcoholism, normal or corrected to normal visual acuity, no metal implants and right-handedness (self-reported). All participants gave written informed consent. The experiments conform to the Declaration of Helsinki, and the experimental protocol was approved by the Ethics Committee of the University of Göttingen.

\subsubsection{Visuomotor learning task}

The stimuli were presented via MR-compatible LCD goggles (Resonance Technology, Northridge, USA) using the software Presentation (version 14.9, Neurobehavioral Systems, Albany, NY). During the task periods, the subjects were presented with visual stimuli consisting of two columns on a light green ( $R G B$ code: $R=155, G=206, B=155$ ) background positioned in equal distances from the midline of the goggles-LCD, covering a visual field of $20^{\circ}$ and $30^{\circ}$ in the vertical and horizontal direction, respectively. The height-level of the left column varied at constant speed following a determined pattern (constant throughout the experiment), whereas the height-level of the right column could be controlled by the subjects. The goal of the task was to make the right column mimic the movement of the left column. To change the height-level of the right-column, the participants had to apply pressure on a custom-made air-filled rubber ball held with the right hand. The ball was connected to a sensor, which converted pressure changes into digital signals with adjustable gain. The digital sensor gain was calibrated according to each subject's strength before the beginning of each run by having the subject press the ball as hard as possible. Information regarding the height- 
level of the left and right columns was sampled with a frequency of $20 \mathrm{~Hz}$ throughout the experiment and saved in a text file for posterior analysis. Subjects received feedback on their performance through the colour of the column they controlled: it was green if the pixel difference between columns was below 40 pixels, yellow if the difference was between 40 and 100 pixels and red whenever the difference exceeded 100 pixels.

\subsubsection{Experimental design}

The experiment followed a block design and consisted of 3 runs, with 50 trials each (Figure 1). In each trial there was a task period lasting $4 \mathrm{~s}$ and a rest period lasting $8 \mathrm{~s}$. The experimental session started with acquisition of structural images and functional imaging was performed during the 3 experimental runs. Stimulation was applied for 10 minutes during the first run.

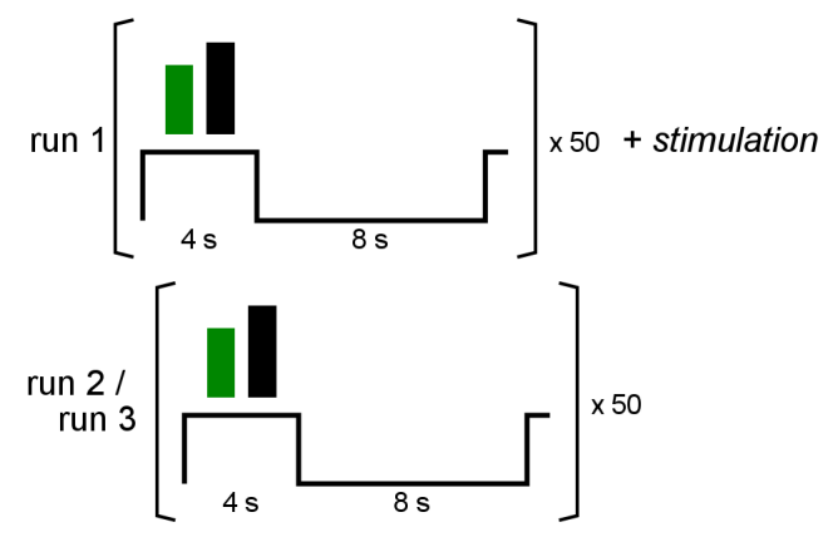

Figure 1. Experimental paradigm. The experimental session included 3 runs, each consisting of 50 trials. In each trial, the task block had a duration of $4 \mathrm{~s}$ followed by a block of $8 \mathrm{~s}$ rest. The stimulation (sham, anodal tDCS, cathodal tDCS, hf-tRNS or If-tRNS) was applied throughout the first run.

\subsubsection{Stimulation}

Stimulation was applied with a battery-driven stimulator (Version DC-Stimulator-Plus, NeuroConn GmbH, Ilmenau, Germany), using a pair of rubber electrodes with dimensions of $5 \times 7 \mathrm{~cm}$ in a previously described set-up compatible with MR environment (Antal et al., 2011). In brief, the electrodes are connected to a filter box, and then to a cable that goes through the wall of the scanner room. Outside the scanner room, the cable is connects with another filter box, to which the stimulator is connected. To prevent heating under the electrodes, the electrode wires were equipped with resistors. 
The intensity of the applied current was $1 \mathrm{~mA}$, with $20 \mathrm{~s}$ fade in and $10 \mathrm{~s}$ fade out in order to minimize sensory perception. The typical montage for tDCS application was used, with one electrode placed over the M1 and another over the contra-lateral forehead. In the anodal tDCS group, the positive electrode was placed over the left M1 and the opposite for the cathodal tDCS group. In the sham group, stimulation consisted solely of 20 seconds fade in and 10 seconds fade out, which provides effective blinding at such stimulation intensity. Regarding tRNS, the frequency spectrum was divided into two ranges: low-frequency (o.1$100 \mathrm{~Hz})$ and high-frequency $(101-640 \mathrm{~Hz})$. The subjects were blinded with regard to the type of stimulation, but the experimenters were not.

\subsubsection{MRI acquisition}

Imaging data was acquired at $3 \mathrm{~T}$ (Magnetom TIM Trio, Siemens Healthcare, Erlangen, Germany) using a standard eight-channel phased array head coil. Subjects were placed supine inside the magnet bore and wore headphones for noise protection. T1-weighted structural images were obtained using a 3D turbo fast low angle shot (FLASH) MRI sequence with $1 \mathrm{~mm} 3$ isotropic resolution (repetition time $(\mathrm{TR})=1950 \mathrm{~ms}$, inversion time $=1100 \mathrm{~ms}$, echo time $(\mathrm{TE})=3.93 \mathrm{~ms}$, flip angle $\left.=12^{\circ}\right)$. For BOLD functional images a multi-slice $\mathrm{T}^{*}{ }^{*}$ sensitive echo-planar imaging (EPI) sequence $\left(\mathrm{TR}=2000 \mathrm{~ms}, \mathrm{TE}=36 \mathrm{~ms}\right.$, flip angle $=70^{\circ}$ was used at a resolution of $262 \mathrm{~mm} 2$. Twenty-two consecutive $4 \mathrm{~mm}$-thick slices angulated in an axial-to-coronal orientation, covering the brain areas of interest (M1, SMA, occipital lobe, basal ganglia and cerebellum) were acquired.

\subsubsection{Analysis of behavioural data}

The tracking error was calculated for each trial, defined as the difference between the required and the applied pressure. The first trial of each run was ignored. The tracking error was averaged for every 5 consecutive trials and normalized with respect to the second trial, to compensate for baseline differences between groups. Statistical analysis was carried out using IBM SPSS Statistics, version 20. A $30 \times 5$ repeated measures analysis of variance (ANOVA) was performed with block (within-subject) and stimulation (between-subject) as factors, both on the normalized and raw tracking error scores. Greenhouse-Geisser corrections were applied, when sphericity assumptions were violated according to Mauchly's test of Sphericity. 
Additionally, to increase SNR of the behavioural data, and to analyse behavioural data in a comparable way to that of the fMRI analysis, the tracking errors were averaged within each run and a $3 \times 5$ repeated measures ANOVA, with run $\times$ stimulation condition as factors was performed.

\subsubsection{Analysis of imaging data}

The analysis of fMRI data was carried out using FEAT (FMRI Expert Analysis Tool) Version 5.98, part of FSL (FMRIB's Software Library, www.fmrib.ox.ac.uk/fsl). For the preprocessing of functional datasets, the following steps were carried out: motion correction (Jenkinson et al., 2002); non-brain removal (Smith, 2002); slice timing correction, spatial smoothing (Gaussian kernel, $8 \mathrm{~mm}$ FWHM); mean-based intensity normalization of all volumes by the same factor; and high-pass temporal filtering (Gaussian-weighted least squares straight line fitting, $15 \mathrm{~s}$ cut-off). Each subject's functional datasets were registered to the T1-weighted structural image and to the MNI152 standard template using FLIRT (Jenkinson and Smith, 2001; Jenkinson et al., 2002). The time-series of each dataset was analysed using a General Linear Model (GLM) approach with autocorrelation correction (Woolrich et al., 2001). The hemodynamic response function (HRF) was modelled as a Gamma variate ( phase $=0$; standard deviation $=3 \mathrm{~s}$, mean lag $=6 \mathrm{~s}$ ).

For the first-level analysis, one explanatory variable (EV) was defined as a square function representing the on-off periods of the task to model motor-related activity (Mov-Rest) and a second EV was defined using the behavioural scores of each participant, orthogonalized with respect to the first EV, to model performance-related activity. Additionally, the six motion parameters calculated during head motion correction were added as covariates of no interest to remove potential signal variability caused by non-corrected motion and the temporal derivatives were also included in the model.

At the second-level, a fixed-effects analysis was performed by averaging the 3 runs of each subject. These were afterwards used to calculate global average activation in a higher-level analysis, for each of the first-level contrasts.

To investigate the effects of stimulation, the results of the second-level analysis were entered into a third-level mixed-effects analysis modelling an ANOVA. In an exploratory manner, and considering that an ANOVA including all the groups might not be sensitive enough to detect changes, if they happen in only one of the groups, we performed 2 separate ANOVAs for tDCS 
and tRNS. Since the type of stimulation is a between-subjects factor and the run is a withinsubjects factor, the effect of run and interaction between run and stimulation condition were calculated separately by entering each first-level analysis into a repeated measured ANOVA regressing out each subject's average activity. Each pair of runs (run1-run2, run1-run3, run2run3) was then contrasted to evaluate activity changes related to motor learning in each step of the experiment.

The probability Z-maps were thresholded with clusters determined by $Z>2.3$ and a significance threshold $\mathrm{P}=0.05$ with (cluster) correction for multiple comparisons, for all analysis described above. 


\subsection{Results}

\subsubsection{Behavioural performance}

Averaging across the 50 subjects, the reduction of tracking error was of approximately 50\% $(53 \pm 13 \%)$ by the end of the experiment. Learning was more pronounced in the first run, at the end of which subjects had increased their performance by an average of $40 \%(41 \pm 14 \%)$, followed by slight gradual improvements throughout the second and third runs (Figure 2). The learning is reflected in the significant effect of block found with the ANOVA $\mathrm{F}(7.43,334.55)=88.322 ; \mathrm{p}<0.001)$. Post-hoc t-tests within the first run (Table 1) showed that significant improvement between consecutive blocks occurred until block 3 for the sham and lf-tRNS groups, and until block 4 for the hf-tRNS and tDCS groups.

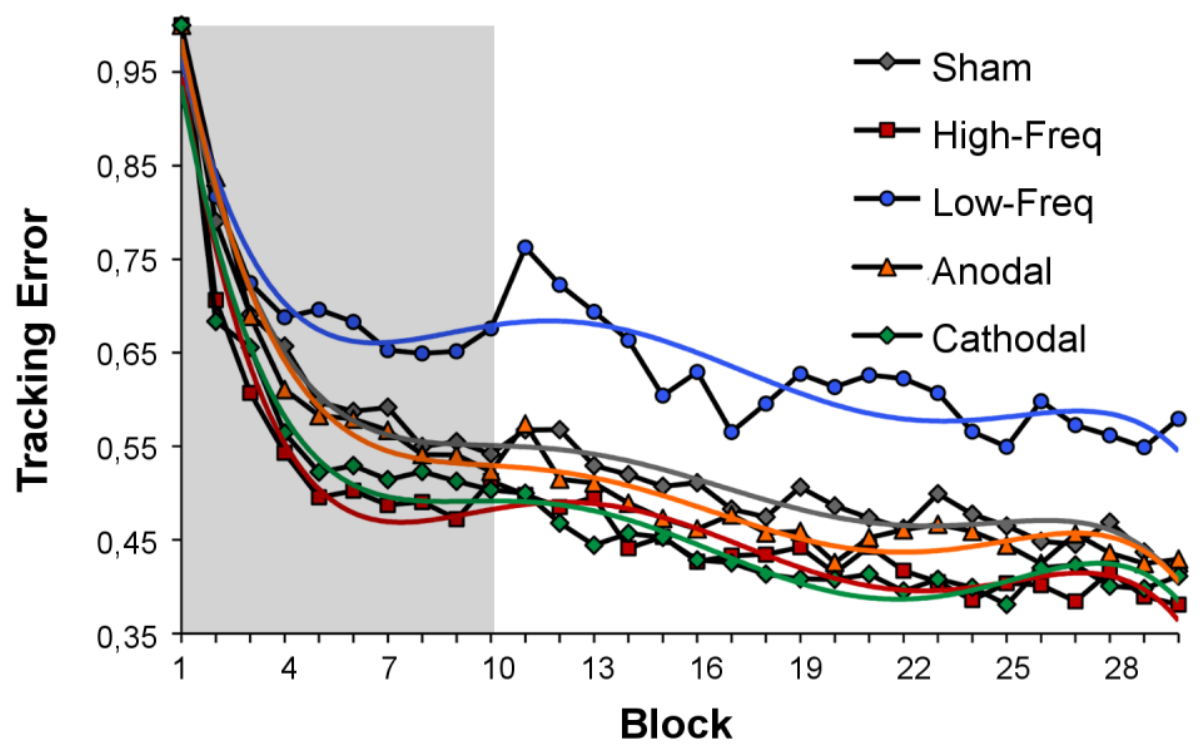

Figure 2. Changes in tracking error relative to the first trial. Shaded area corresponds to the stimulation period $(10 \mathrm{~min})$. Fifth polynomial trendlines are superimposed on the data for easier visualization.

There was no significant effect of stimulation $\left(\mathrm{F}_{(4,45)}=1.464 ; \mathrm{p}=0.115\right)$ or interaction of block and stimulation $\left(\mathrm{F}_{(116)}=0.641 ; \mathrm{p}=0.999\right)$. However, there was a tendency for poorer learning in the group receiving lf-tRNS and better learning in the groups receiving cathodal tDCS and hf-tRNS. Similarly, when performance was averaged for the whole run, the ANOVA revealed significant effect of run $\left(\mathrm{F}_{(1.72,77.43)}=101.776 ; \mathrm{p}<0.001 ;\right)$ but no significant effect regarding stimulation condition $\left(\mathrm{F}_{(4,45)}=1.128 ; \mathrm{p}=0.355 ;\right)$ or run $\times$ stimulation interaction $\left(\mathrm{F}_{(8)}=0.391\right.$; $\mathrm{p}=0.923 ;)$. 
Table 1. Post-hoc t-tests between consecutive blocks of the first run.

\begin{tabular}{lcccccccccc}
\hline & \multicolumn{2}{c}{ Sham } & \multicolumn{2}{c}{ anodal tDCS } & \multicolumn{2}{c}{ cathodal tDCS } & \multicolumn{2}{c}{ hf-tRNS } & \multicolumn{2}{c}{ If-tRNS } \\
\hline Blocks & $\mathbf{T}$ & $\mathbf{p}$ & $\mathbf{t}$ & $\mathbf{p}$ & $\mathbf{t}$ & $\mathbf{p}$ & $\mathbf{t}$ & $\mathbf{p}$ & $\mathbf{t}$ & $\mathbf{p}$ \\
\hline $1-2$ & $\mathbf{3 . 8 2 5}$ & $\mathbf{0 . 0 0 4}$ & $\mathbf{4 . 0 2 0}$ & $\mathbf{0 . 0 0 3}$ & $\mathbf{8 . 8 2 1}$ & $<\mathbf{0 . 0 0 1}$ & $\mathbf{3 . 9 9 0}$ & $\mathbf{0 . 0 0 3}$ & $\mathbf{4 . 8 1 5}$ & $\mathbf{0 . 0 0 1}$ \\
$2-3$ & $\mathbf{2 . 7 9 9}$ & $\mathbf{0 . 0 2 1}$ & $\mathbf{3 . 6 1 7}$ & $\mathbf{0 . 0 0 6}$ & 1.308 & 0.223 & 2.088 & 0.066 & $\mathbf{4 . 4 8 5}$ & $\mathbf{0 . 0 0 2}$ \\
$3-4$ & 1.050 & 0.321 & $\mathbf{2 . 3 2 1}$ & $\mathbf{0 . 0 4 5}$ & $\mathbf{4 . 3 1 9}$ & $\mathbf{0 . 0 0 2}$ & $\mathbf{2 . 3 7 0}$ & $\mathbf{0 . 0 4 2}$ & 1.248 & 0.243 \\
\hline $\mathbf{4}-5$ & 1.904 & 0.089 & 1.217 & 0.255 & 1.228 & 0.251 & $\mathbf{2 . 7 7 4}$ & $\mathbf{0 . 0 2 2}$ & -0.292 & 0.777 \\
$5-6$ & 0.462 & 0.655 & 0.170 & 0.868 & -0.228 & 0.825 & -0.414 & 0.689 & -0.030 & 0.977 \\
\hline $6-7$ & -0.099 & 0.924 & 0.305 & 0.767 & 0.832 & 0.427 & 1.147 & 0.281 & 1.250 & 0.243 \\
$7-8$ & 1.835 & 0.100 & 1.029 & 0.330 & -0.379 & 0.714 & -0.132 & 0.898 & 0.498 & 0.630 \\
\hline $\mathbf{8}-9$ & -0.109 & 0.915 & 0.027 & 0.979 & 0.482 & 0.641 & 0.957 & 0.363 & -0.969 & 0.358 \\
$9-10$ & 0.645 & 0.535 & 0.867 & 0.408 & 0.200 & 0.846 & -1.492 & 0.170 & -0.498 & 0.630 \\
\hline
\end{tabular}

In addition, we repeated the analysis for non-normalized data (Table 2). Here we found a significant effect of block $\left(\mathrm{F}_{(6.59,296.67)}=71.025 ; \mathrm{p}<0.001\right)$ or $\operatorname{run}\left(\mathrm{F}_{(1.71,76.84)}=96.975 ; \mathrm{p}<0.001\right)$, and a significant effect of stimulation $\left(\mathrm{F}_{(4,45)}=3.566 ; \mathrm{p}=0.013\right.$ on the analysis by block and $\mathrm{F}_{(4,45)}=4.024 ; \mathrm{p}=0.007$ on the analysis by run). The interactions of block $\times$ stimulation and run $\times$ stimulation were non-significant $\left(\mathrm{F}_{(116)}=0.833 ; \mathrm{p}=0.897\right.$ and $\mathrm{F}_{(8)}=0.469 ; \mathrm{p}=0.875$, respectively). The stimulation effect is due to the better initial performance of the cathodal tDCS and hf-tRNS groups.

Table 2. Statistical analysis of the non-normalized behavioural data.

\begin{tabular}{lcc}
\hline & $\mathbf{F}$ & $\mathbf{p}$ \\
\hline Block $\times$ Stimulation & & \\
\hline block & $\mathbf{7 1 . 0 2 5}$ & $<\mathbf{0 . 0 0 1}$ \\
stimulation & $\mathbf{3 . 5 6 6}$ & $\mathbf{0 . 0 1 3}$ \\
block $\times$ stimulation & 0.833 & 0.897 \\
Run $\times$ Stimulation & & \\
\hline run & $\mathbf{9 6 . 9 7 5}$ & $<\mathbf{0 . 0 0 1}$ \\
stimulation & $\mathbf{4 . 0 2 4}$ & $\mathbf{0 . 0 0 7}$ \\
run $\times$ stimulation & 0.469 & 0.875 \\
\hline
\end{tabular}

\subsubsection{Imaging results}

The task activated an extensive network including the M1 and premotor cortex, SMA, prefrontal and occipital cortical areas, as well as the thalamus and basal ganglia (see Table 3 for coordinates of main clusters). The performance-related network was similar, but less extensive and did not include the M1 and premotor cortex (Table 4). 
Table 3. Peak voxel intensity and coordinates for brain regions showing motor task-related activity.

\begin{tabular}{lcccc}
\hline & \multicolumn{4}{c}{ MNI Coordinates } \\
\hline Anatomical Region & $\boldsymbol{Z}$ & $\boldsymbol{x}$ & $\boldsymbol{y}$ & $\boldsymbol{z}$ \\
\hline L precentral gyrus & 8,9 & -58 & 6 & 22 \\
R precentral gyrus & 9,6 & 54 & 10 & 20 \\
\hline L postcentral gyrus & 9,5 & -4 & 2 & 6 \\
\hline L supplementary motor area & 9,5 & -4 & 2 & 46 \\
\hline R middle frontal gyrus & 9,9 & 38 & 0 & 50 \\
\hline L prefrontal cortex & 3,9 & -36 & 38 & 4 \\
\hline R prefrontal cortex & 6,8 & 38 & 42 & 22 \\
\hline anterior cingulate cortex & 9,9 & 8 & 16 & 34 \\
\hline L temporal occipital fusiform cortex & 9,5 & -22 & -78 & -14 \\
\hline R temporal occipital fusiform cortex & 9,9 & 22 & -74 & -18 \\
\hline L lateral occipital cortex (inf) & 9,8 & -44 & -78 & 2 \\
\hline R lateral occipital cortex (inf) & 10,1 & 48 & -76 & 0 \\
\hline L occipital pole & 10,2 & -2 & -90 & 16 \\
\hline R occipital pole & 8,8 & 18 & -94 & 18 \\
\hline L putamen & 9,3 & -22 & 8 & 2 \\
\hline R putamen & 9,7 & 24 & 10 & -2 \\
\hline R pallidum & 8,9 & 16 & 6 & 0 \\
\hline L pallidum & 8,7 & -18 & -2 & -2 \\
\hline L thalamus & 9,8 & -14 & -22 & 4 \\
\hline R thalamus & 6,5 & 10 & -20 & 6 \\
\hline
\end{tabular}

\section{Temporal changes}

A decrease in motor task-related activation was observed in the premotor cortex, M1, SMA, left LOC, left temporal occipital fusiform cortex and basal ganglia (Figure 3A) during time (with the contrast run1-run2). The contrast run2-run3 revealed that activation continued to decrease in areas comprising the precuneous, superior parietal cortex, middle and inferior frontal gyrus, right prefrontal cortex, left inferior lateral occipital cortex (LOC) and basal ganglia (Figure 3B). The only regions showing increased activation was the PCC, as observed with contrast run3-run1 (Figure 4). Concerning performance-related activity, contrast run1run2 revealed that activation of the paracingulate gyrus, superior frontal gyrus, thalamus and hippocampus decreased from the first to the second run (Figure 5). No significant changes were detected with the other contrasts. 


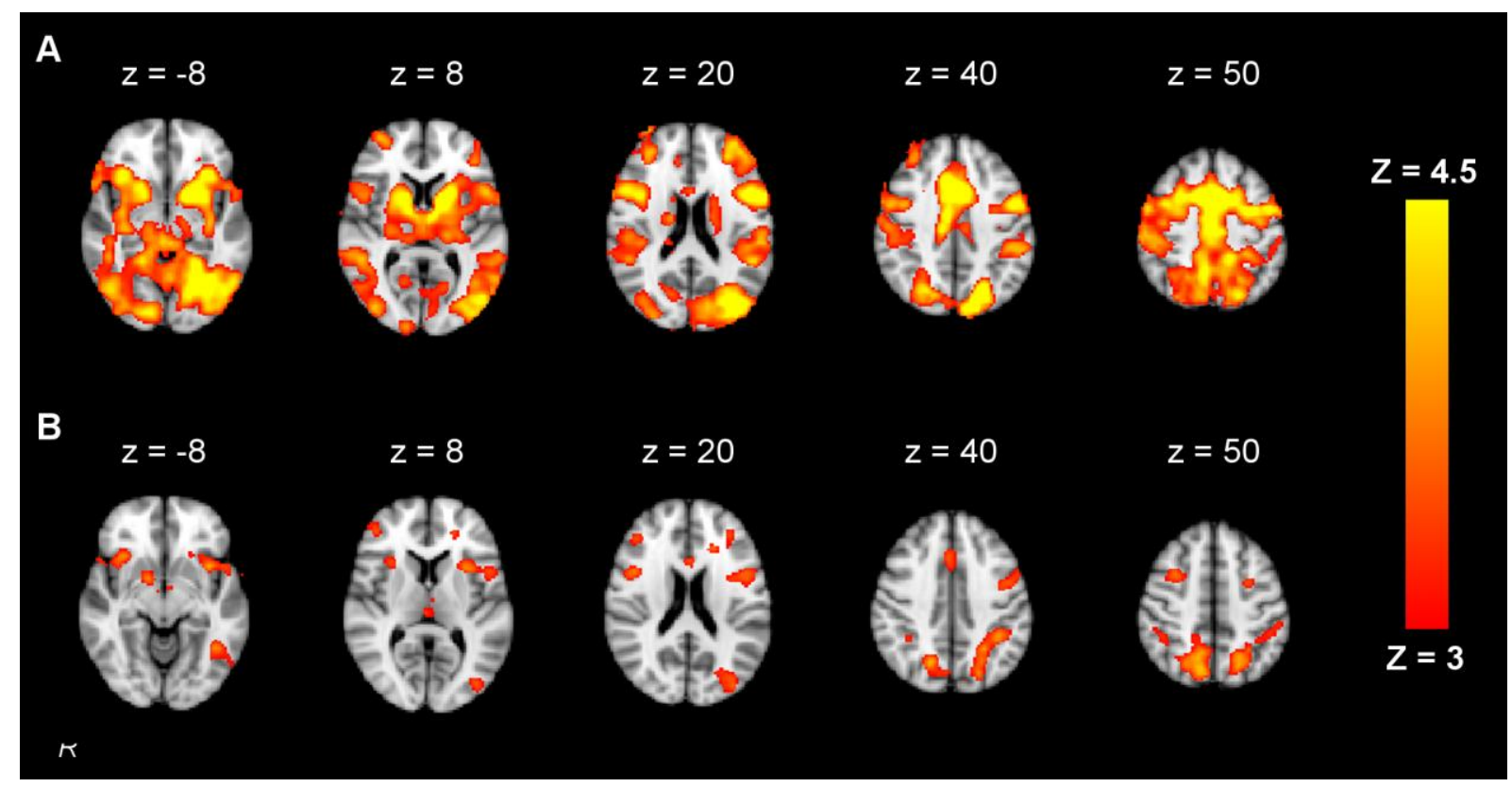

Figure 3. Motor task-related decrease of brain activity during and after stimulation. Activity decreased with time for contrasts A) run1-run2 in primary and premotor cortices, supplementary motor area (SMA), prefrontal cortex, occipital cortex, thalamus and basal ganglia and B) run2-run3 in the precuneous, superior parietal cortex, middle and inferior frontal gyrus, right prefrontal cortex, left inferior LOC and basal ganglia $(Z>3, P<0.05$, corrected).

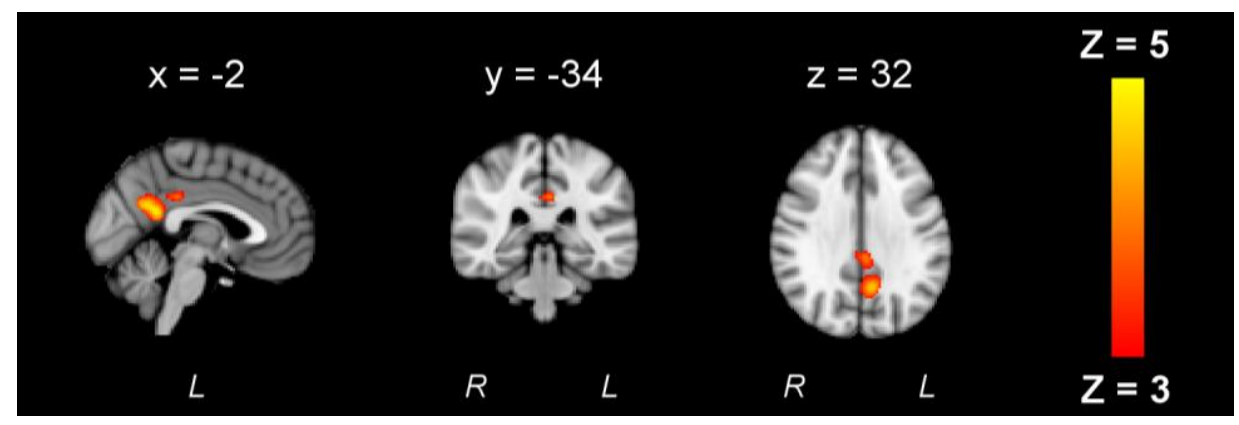

Figure 4. Motor task-related increase of brain activity during and after stimulation. Average of brain regions showing an increase in activity in the PCC with time for contrast run3-run1 $(Z>3$, $\mathrm{P}<0.05$, corrected).

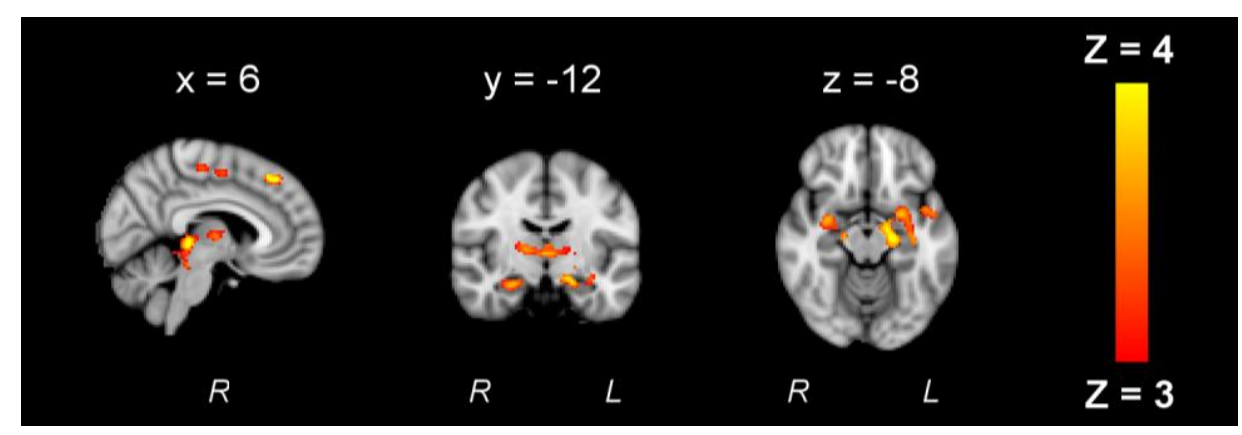

Figure 5. Performance related decrease of brain activity during and after stimulation. Activity decreased with time for contrast run1-run2 in the paracingulate gyrus, superior frontal gyrus, thalamus and hippocampus. ( $Z>3, P<0.05$, corrected). 
Table 4. Peak voxel intensity and coordinates for brain regions showing performancerelated activity.

\begin{tabular}{lcccc}
\hline & \multicolumn{5}{c}{ MNI Coordinates } \\
\hline Anatomical Region & $\boldsymbol{Z}$ & $\boldsymbol{x}$ & $\boldsymbol{y}$ & $\boldsymbol{z}$ \\
\hline L precentral gyrus & 6,5 & -44 & 0 & 42 \\
\hline R postcentral gyrus & 5,7 & 54 & -20 & 50 \\
\hline supplementary motor area & 5,9 & -2 & 8 & 56 \\
\hline L superior frontal gyrus & 5,4 & -4 & 34 & 42 \\
\hline R middle frontal gyrus & 5,8 & 44 & 6 & 52 \\
\hline L middle frontal gyrus & 6,4 & -48 & 10 & 32 \\
\hline R inferior frontal gyrus & 7,4 & 54 & 32 & -6 \\
\hline L inferior frontal gyrus & 6,4 & -50 & 18 & -4 \\
\hline L prefrontal cortex & 4,8 & -28 & 54 & 24 \\
\hline R prefrontal cortex & 4,2 & 30 & 54 & 28 \\
\hline anterior cingulate cortex & 5 & 4 & 20 & 28 \\
\hline L middle temporal gyrus & 6,2 & -62 & -46 & -4 \\
\hline R insula & 4,4 & 36 & -20 & 6 \\
\hline L temporal occipital fusiform cortex & 6,9 & -26 & -52 & -14 \\
\hline R occipital fusiform cortex & 5,8 & 30 & -84 & -16 \\
\hline L lateral occipital cortex & 6,7 & -38 & -86 & 22 \\
\hline R lateral occipital cortex & 6,1 & 40 & -76 & 32 \\
\hline Cuneous & 6,6 & 0 & -88 & 24 \\
\hline Precuneous & 5,9 & 6 & -50 & 52 \\
\hline
\end{tabular}

\section{Stimulation changes}

When including all the stimulation conditions in one ANOVA, no significant effect of stimulation was detected. However, a separate ANOVA including only hf-tRNS, lf-tRNS and sham groups showed that stimulation affected task-related activity. In particular, in the hftRNS group activation of the left frontal cortex $(x=-44, y=28, z=18)$ decreased when compared to sham (Figure 6A), and decreased in the left frontal cortex $(x=-44, y=32, z=12)$, precuneous $(\mathrm{x}=4, \mathrm{y}=-72, \mathrm{z}=32)$ and right frontal cortex $(\mathrm{x}=38, \mathrm{y}=26, \mathrm{z}=52)$ when compared to the lf-tRNS group (Figure 6B). No significant changes in performance-related activity were found due to tRNS and no changes in motor-related or performance-related activity were found due to tDCS. 


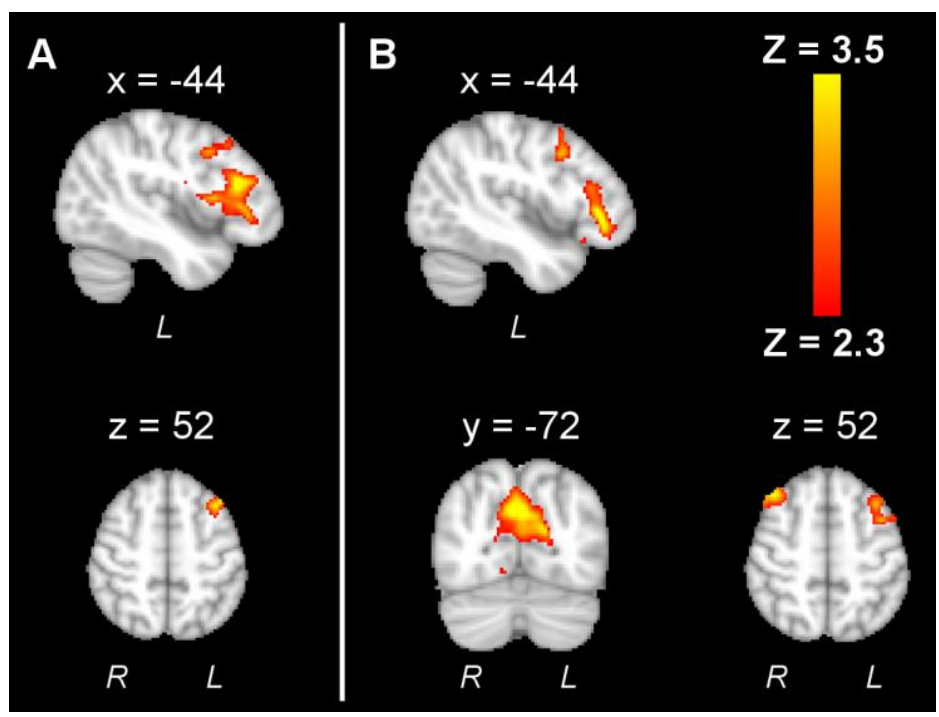

Figure 6. Regions of decreased activity for hf-tRNS. A) Contrast sham - hf-tRNS revealed changes in the left frontal cortex. B) Contrast If-tRNS - hf-tRNS revealed additional changes in right frontal cortex and precuneous.

\section{Image quality}

To confirm that image quality was not significantly affected by the stimulation we have performed voxelwise statistics on the mean BOLD signal. Mean EPI images for each subject were calculated by averaging the 3 runs. Then, non-parametric statistics were performed using the tool randomise from FSL, to test for systematic differences caused by stimulation. Shown are the results of an F-test with voxelwise thresholding at significance level $\mathrm{p}=0.05$. The found differences are punctual, do not relate to electrode location and do not correspond to brain regions where significant changes due to stimulation were found. (Figure 7).

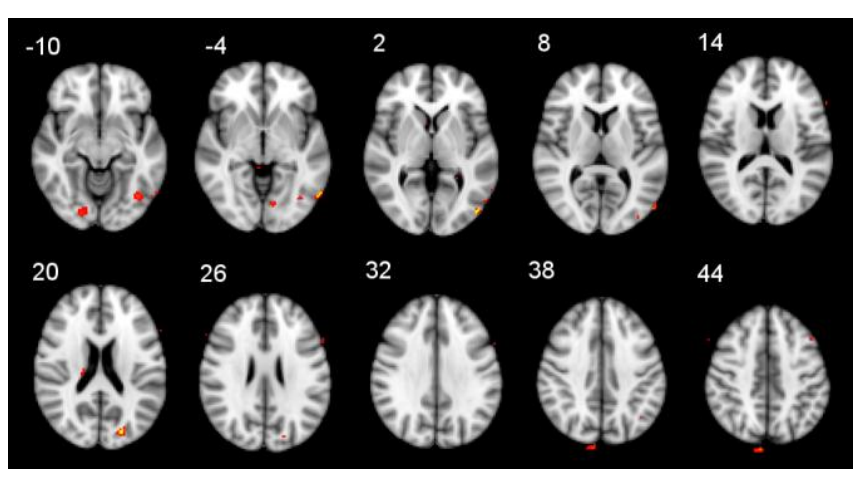

Figure 7. Voxels showing significant effect of stimulation condition on the mean BOLD signal. Mean EPI images were averaged across runs for each subject and then non-parametric statistics were performed to test for systematic differences caused by stimulation. Shown are the results of an F-test with voxelwise thresholding at significance level $p=0.05$. 


\subsection{Discussion}

\subsubsection{General learning and associated brain activity}

In this study we have replicated the well established motor learning behaviour, representing an initial stage of fast skill acquisition, then followed by a stage where learning occurs via slow gradual improvement (see, for instance, Dayan and Cohen, 2011; Doyon and Benali, 2005). According to the results, the fast learning stage ended after 3 to 4 blocks (up to 20 trials). Regarding the associated BOLD brain activity detect by fMRI we found a general decrease in the task-related network, in the M1, SMA, primary somatosensory cortex, premotor cortex, prefrontal cortex, frontal gyrus, insula, anterior cingulate cortex (ACC) and precuneous. Such activation reductions in motor related areas have been described previously in the literature. An increase in movement automaticity can be the explanation for the reduced activation of the ACC as suggested by previous work (Floyer-Lea and Matthews, 2004) and by the role of this region in effector functions and attention (Vogt et al., 1992). However, the results also show that towards the end of the experiment, the posterior cingulate cortex increased activity. Such apparently contradictory result can be explained by the functional heterogeneity of the cingulate cortex (Beckmann et al., 2009), with the posterior portion being mainly involved in spatial orientation and memory (Vogt et al., 1992). The observed changes in performance-related activity within the prefrontal cortex are in agreement with our behavioural results and with previous work using the same task (FloyerLea and Matthews, 2004), as it decreased from the first to the second runs and then stabilized.

\subsubsection{Effects of stimulation}

Surprisingly, neither tDCS nor tRNS resulted in significant modulation at a behavioural level. Nevertheless, cathodal tDCS and hf-tRNS showed a tendency to improve learning, whereas lf-tRNS seemed to slightly impair the learning process. Concerning the relative direction of the effects of the different stimulation conditions, our results somewhat reflect previously observed changes in M1 excitability: even though hf-tRNS and cathodal tDCS lead to opposite changes in brain excitability when applied during rest (cathodal reduces and tRNS increases), during a motor task (compressing a rubber ball with the right hand), both types of stimulation lead to excitability decrease (Antal et al., 2007; Terney et al., 2008). Previous 
studies where lf-tRNS was used have found little to no effect of lf-tRNS, both in excitability alterations and cognitive tasks (Fertonani et al., 2011; Terney et al., 2008).

It is possible that the outcome measure of the task we used is not sensible enough to detect potentially small effects of the stimulation. We used a task that requires complex integration of visual and motor information and an outcome measure depending on fine precision of movements, whereas previous work found, for instance, an effect of tDCS on reaction times of a simple task requiring learning of a sequence of finger movements, improved by anodal and slowed by cathodal tDCS over the M1 (Stagg et al., 2011). Another positive effect of anodal tDCS was the improvement of skill acquisition in a sequential visual isometric pinch task, based on speed accuracy trade-off (Reis et al., 2009), but a straightforward comparison of our behavioural results with previously reported data is prevented by differences in study design, such as timing of stimulation, which can be crucial for the outcome of an experiment in terms of both brain excitability and behavioural measures (Antal et al., 2008a; Stagg et al., 2011; Terney et al., 2008). Also, previous studies of visuomotor tasks have used other stimulation montages, which might have been more effective in our case as well. For instance, it was reported that cathodal tDCS had no effect on learning whereas anodal stimulation facilitated learning both with a M1 or V5-Cz montage (Antal et al., 2004a). However, only $\mathrm{V}_{5}$-Cz montage was effective in modulation of performance after stabilization; after the task was learned, cathodal tDCS improved and anodal tDCS worsened performance (Antal et al., 2004b). Thus, it is possible that also in our paradigm a $\mathrm{V}_{5}-\mathrm{Cz}$ montage would prove more effective in modulation of learning or performance, which should be addressed in future studies.

Regarding changes in brain activity, the results show activity reduction in the visual cortex, precuneous and left pFC when comparing hf-tRNS with lf-tRNS and sham. There are very few studies combining fMRI with tRNS and one of them found that during finger movements, activity was decreased in the M1 due to the stimulation. However, recent research so far points in the direction of a functional specialization of the effects of the stimulation, in detriment of strict dependence on the stimulation site (Reis and Fritsch, 2011). Contrary to a simple finger movement, the task that was used requires an integration of visual and motor information for the successful acquisition of skill and subsequent improvement. The role of precuneus has been implicated in motor coordination as well as processing of visuospatial information and attentive tracking, and displays connections to the prefrontal cortex, dorsal 
premotor cortex and SMA (Cavanna and Trimble, 2006), thus partially explaining our results.

\subsubsection{Study limitations}

The absence of effects on behavioural performance caused by stimulation is surprising in light of results described in the literature. One of the limitations of this study, which may have prevented the detection of a positive effect of tDCS and tRNS, is the small sample size, in comparison to previous studies reporting a significant effect of stimulation but using different experimental paradigms (Antal et al., 2004b; Fertonani et al., 2011; Terney et al., 2008).

Another limitation derives from the fact that the initial performance levels were not the same across all of the stimulation groups, as made evident by the analysis of the non-normalized data (Table 2). Both the cathodal tDCS and hf-tRNS groups were initially better and were able to achieve greater improvement than the other groups. Therefore, we cannot say whether the stimulation-related improvements in learning are due to the subsequent ability to learn better. On the other hand, the stimulation can have immediate effects, which in this case, are not separable from the native initial performance differences. Future studies should therefore, investigate balanced groups concerning initial performance levels. 


\section{Project 2 - Improvement of fatigue symptoms in patients with multiple sclerosis with anodal tDCS}

The goal of this project was to assess whether fatigue symptoms in patients with MS can be reduced by facilitatory tDCS over the left DLPFC.

As presented in the introductory section, current research has so far not been able to establish clear anatomical landmarks for fatigue in MS. Nevertheless, the role of the frontal lobe in the development of fatigue symptoms is recurrent across the literature. Concerning structural alterations, fatigue has been associated with increased left frontal lesion load (Sepulcre et al., 2009) and diffusion abnormalities (Pardini et al., 2010) in left frontal white matter and within anterior thalamic tracts (Bester et al., 2013), as well as with atrophy of the left superior frontal and left precentral gyri (Riccitelli et al., 2011a; Sepulcre et al., 2009). Furthermore, reduction of glucose metabolism in the frontal cortex (Roelcke et al., 1997), added to the observations of a fMRI study of reduced left frontal activation during a motor task (Filippi et al., 2002), provide evidence of a role of a dysfunctional frontal cortex in MS patients suffering from fatigue.

Based on the results of previous studies (Felipe Fregni et al., 2006b; Monte-Silva et al., 2013), we tested two stimulation protocols and measured changes in fatigue with several clinically acknowledged questionnaires, while simultaneously monitoring depression levels and using MRI to control the occurrence of new lesions.

Considering that anodal tDCS is able to enhance cortical excitability and its application over the left prefrontal cortex has been demonstrated to improve cognitive functions, both in healthy populations and in patients suffering from neuropsychiatric diseases (Flöel, 2013; Kuo and Nitsche, 2012; M.-F. Kuo et al., 2013), we expected that it would be an effective intervention in decreasing the levels of fatigue.

The results of protocol 1 have been accepted for publication in Restorative Neurology and Neuroscience under the title: "Impact of transcranial direct current stimulation on fatigue in multiple sclerosis “ (Saiote et al., 2014). 


\subsection{Methods}

\subsubsection{Subjects}

All patients were recruited from the outpatient pool of our department and were diagnosed with clinically definite MS.

Inclusion criteria were relapsing-remitting disease course, minimum of 2 months since the last relapse, right-handedness, FSS $\geq 4$, fatigue persisting for at least 8 weeks, no depression (Beck's Depression Inventory (BDI) < 19), Expanded Disability Status Scale (EDSS) $\leq 6$, no paresis of the upper limbs and no central nervous system-active medication besides MS basic therapy. All aspects of the study conformed to the Declaration of Helsinki, and were approved by the Ethics Committee of the University of Goettingen. Clinical and demographic characteristics of the patients who took part in the study are listed in Table 5 .

\section{Protocol 1}

Fourteen patients were enrolled in the study for protocol 1. One patient was excluded after the first block due to insufficient fatigue level (FSS $<4)$. Thirteen patients were included in the analysis (10 female, age: $46.8 \pm 6.8$, range $37-59$ ). Ten patients were receiving disease modifying therapy with interferon beta $(\mathrm{N}=3)$, glatiramer acetate $(\mathrm{N}=4)$ or natalizumab $(\mathrm{N}=3)$.

\section{Protocol 2}

Eleven patients took part in the study. Two patients dropped out and one was excluded due to experimental problems. Therefore, 8 patients were included in the complete analysis (5 female, age: $44.4 \pm 4.0$, range $28-58$ ). Seven patients were receiving disease modifying therapy with glatiramer acetate $(\mathrm{N}=5)$ or natalizumab $(\mathrm{N}=2)$. Four patients participated in both protocols.

\subsubsection{Assessment of fatigue}

For assessing fatigue, patients filled in the FSS, the MSFSS and the MFIS on every session. The full fatigue questionnaires are presented in Appendix 1. The FSS consists of 9 items, each to be rated in a Likert scale of 1-7. It mainly concerns physical aspects of fatigue and how strong is their interference with daily life. The MSFSS consists of 5 descriptive items related to MS-specific characteristics of fatigue, such as variation according to temperature and 
relationship with mood, also to be rated in a Likert scale from 1-7. The MFIS consists of 21 items to be rated from o (never) to 4 (almost always), which can be divided in subscales measuring the physical (MFISphy), cognitive (MFIScog) and psychosocial (MFISpsych) impact of fatigue. In addition, at all sessions after stimulation (Figure 9) patients were asked to rate the change in perceived fatigue compared to baseline in a scale from -5 to 4 , where the meaning of each value was the following: -5 : complete withdrawal (of fatigue symptoms); -4 : very strong decrease; -3: strong decrease; -2: moderate decrease; -1: slight decrease; 0 : no change; 1: slight increase; 2: moderate increase; 3: strong increase; and 4: very strong increase. For protocol 2, patients were additionally asked to rate fatigue, and also quality of life, resilience, daily energy, mood and vigour using a Visual Analog Scale (VAS) rated from $0-10 \mathrm{~cm}$.

To monitor depression, patients completed the BDI and Hamilton's Anxiety and Depression Scale (HADS) on every session.

Table 5. Individual demographic and clinical characteristics of the patients

\begin{tabular}{|c|c|c|c|c|c|c|c|}
\hline Patient & Age & Sex & DMT & EDSS & MS duration & FSS & BDI \\
\hline \multicolumn{8}{|c|}{ Protocol 1} \\
\hline 1 & 43 & $\mathrm{~F}$ & interferon $\beta$ & 2 & 3 & 6.78 & 7 \\
\hline 2 & 43 & $\mathrm{~F}$ & - & 2 & 10 & 4 & 3 \\
\hline 3 & 47 & $\mathrm{~F}$ & glatiramer acetate & 3.5 & 4 & 5.5 & 6 \\
\hline 4 & 37 & $\mathrm{~F}$ & glatiramer acetate & 2 & 7 & 5.89 & 7 \\
\hline 5 & 46 & $\mathrm{~F}$ & interferon $\beta$ & 3.5 & 7 & 6.67 & 13 \\
\hline 6 & 53 & $M$ & natalizumab & 3.5 & 6 & 5.11 & 4 \\
\hline 7 & 40 & $\mathrm{~F}$ & Interferon $\beta$ & 4 & 8 & 6.33 & 4 \\
\hline 8 & 48 & $\mathrm{~F}$ & - & 3 & 19 & 5.67 & 5 \\
\hline 9 & 55 & $\mathrm{~F}$ & glatiramer acetate & 4.5 & 14 & 5.22 & 12 \\
\hline 10 & 37 & M & natalizumab & 6 & 6 & 6 & 2 \\
\hline 11 & 50 & $\mathrm{~F}$ & glatiramer acetate & 2.5 & 5 & 6.11 & 8 \\
\hline 12 & 59 & $\mathrm{~F}$ & - & 2 & 20 & 5.11 & 5 \\
\hline 13 & 51 & $M$ & natalizumab & 5.5 & 8 & 7 & 3 \\
\hline \multicolumn{8}{|c|}{ Protocol 2} \\
\hline 1 & 58 & $\mathrm{~F}$ & glatiramer acetate & 4.5 & 16 & 5.11 & 10 \\
\hline 2 & 48 & $\mathrm{~F}$ & glatiramer acetate & 2 & 5 & 6.22 & 6 \\
\hline 3 & 51 & $\mathrm{~F}$ & glatiramer acetate & 2.5 & 7 & 4.22 & 5 \\
\hline 4 & 38 & M & interferon $\beta$ & 6 & 8 & 5.33 & 0 \\
\hline 5 & 33 & M & interferon $\beta$ & 2 & 5 & 4.89 & 3 \\
\hline 6 & 28 & $M$ & glatiramer acetate & 2.5 & 7 & 5.44 & 9 \\
\hline 7 & 58 & $\mathrm{~F}$ & - & 2 & 20 & 6.11 & 5 \\
\hline 8 & 41 & $\mathrm{~F}$ & glatiramer acetate & 2.5 & 10 & 6.11 & 13 \\
\hline
\end{tabular}




\subsubsection{Stimulation}

TDCS was applied with a battery-driven stimulator (DC-Stimulator Plus, NeuroConn, Germany) with $1 \mathrm{~mA}$ intensity for periods of $20 \mathrm{~min}$. The electrodes were inserted in sponges soaked in saline solution and were kept in the right position with the aid of non-conductive rubber bands. The current was ramped up for $30 \mathrm{sec}$ until reaching the intended intensity of 1 $\mathrm{mA}$ and ramped down for $30 \mathrm{sec}$ at the end of the stimulation period. For sham stimulation, the current was ramped down immediately after ramping up, providing effective blinding at the used intensity (Ambrus et al., 2012; Gandiga et al., 2006).

Patients were questioned with regard to skin sensations during the stimulation and at the end of the experiment, and whether they were able to discern between stimulation conditions. The experimenters were also blinded to the stimulation condition. The anode $(5 \times 7 \mathrm{~cm})$ was placed over the left DLPFC (defined by position F3 of the 10-20 EEG electrode system) and the cathode $(6 \times 15 \mathrm{~cm})$ was placed on the contralateral forehead (Figure $8 \mathrm{~A})$. The size of the cathode was chosen to make it functionally inert (Nitsche et al., 2007).

To simulate the electric field induced by the chosen montage, a finite element head model (healthy male, age=36) was created as described in Opitz et al. (2011) using SimNibs (Windhoff et al., 2013). Five different tissue types including WM, GM, CSF, skin and skull were taken into account and their conductivities were assumed isotropic. The electric field distribution for a current of $1 \mathrm{~mA}$ passing through the electrodes was calculated numerically (Figure 8B), showing the largest electric fields in the prefrontal cortex.

\subsubsection{MRI sessions}

MRI was performed at 3T (Magnetom TIM Trio, Siemens Healthcare, Erlangen, Germany). T1-weighted images were obtained with a $3 \mathrm{D}$ turbo FLASH sagital sequence (TI $=900 \mathrm{~ms}$, flip-angle $=9^{\circ}, \mathrm{TR}=2250 \mathrm{~ms}, \mathrm{TE}=3.26 \mathrm{~ms}$, voxel size $\left.=1 \mathrm{~mm}^{3}\right)$ and T2-FLAIR 3D sequence $\left(\mathrm{TI}=2100 \mathrm{~ms}, \mathrm{TR}=6000 \mathrm{~ms}, \mathrm{TE}=403 \mathrm{~ms}\right.$, voxel size $\left.=1 \mathrm{~mm}^{3}\right)$. Gadolinium-enhanced T1weighted images were obtained to control for the presence of active lesions. 
A

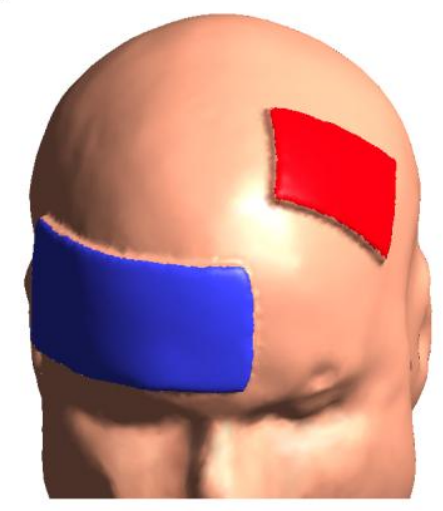

B

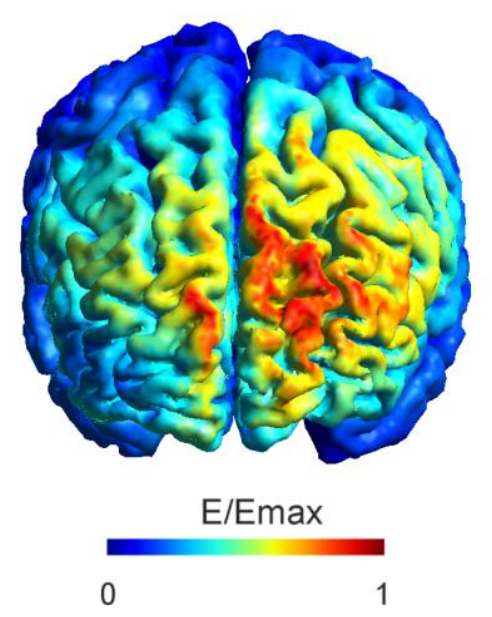

Figure 8. Electrode montage and simulation of the electric field distribution. A) The anode $(5 \times 7$ $\mathrm{cm})$ was placed over F3 and the cathode $(6 \times 15 \mathrm{~cm})$ over the contralateral forehead. B) The simulated electric field distribution shows higher electric field strengths occurring at the left prefrontal cortex.

\subsubsection{Experimental protocol}

We used a crossover design in which all patients underwent 2 blocks in pseudo-randomised and counterbalanced order, one with real, and the other with sham stimulation. Each block started at day o with a baseline session with fatigue and depression questionnaires (Figure 9). A minimum of 2 weeks wash-out period separated the end of the first block from the beginning of the second block. Sessions were performed at approximately the same time of the day (morning, early afternoon or late afternoon) throughout the experiment for each patient, because fatigue levels can change according to time of the day, for instance increasing during the second part of the day (Krupp et al., 2010). The patients were informed that on one of the blocks they would receive real stimulation and placebo stimulation on the other.

\section{Protocol 1}

Stimulation was applied once for 20 min on days 1 to 5 . Afterwards, the patients were again asked to fill in the fatigue and depression questionnaires. On days 8, 10, 15 and 30, follow-up measurements were performed in which patients filled in the questionnaires. Patients underwent an MRI session on day o to ensure the absence of active lesions. Additionally, on day 30 another MRI session was performed in order to control for the occurrence of new lesions during the experiment (Figure 9A). 


\section{Protocol 2}

The stimulation applied twice only on day 1 , with duration of 20 min and with an interval between the two stimulation periods of approximately $20 \mathrm{~min}$. The MRI session to control for active lesions was performed on day 1. Follow-up measurements with the questionnaires were performed on days 2, 4, 6, 11 and 26. Questionnaires from days 2, 6 and 26 were filled in by the patients at home to reduce the frequency with which patients had to come to the hospital, thus reducing the inconvenience caused by taking part in the study. The patients were asked to fill in the questionnaires at the same time as the experimental sessions that took part in the hospital (Figure 9B).

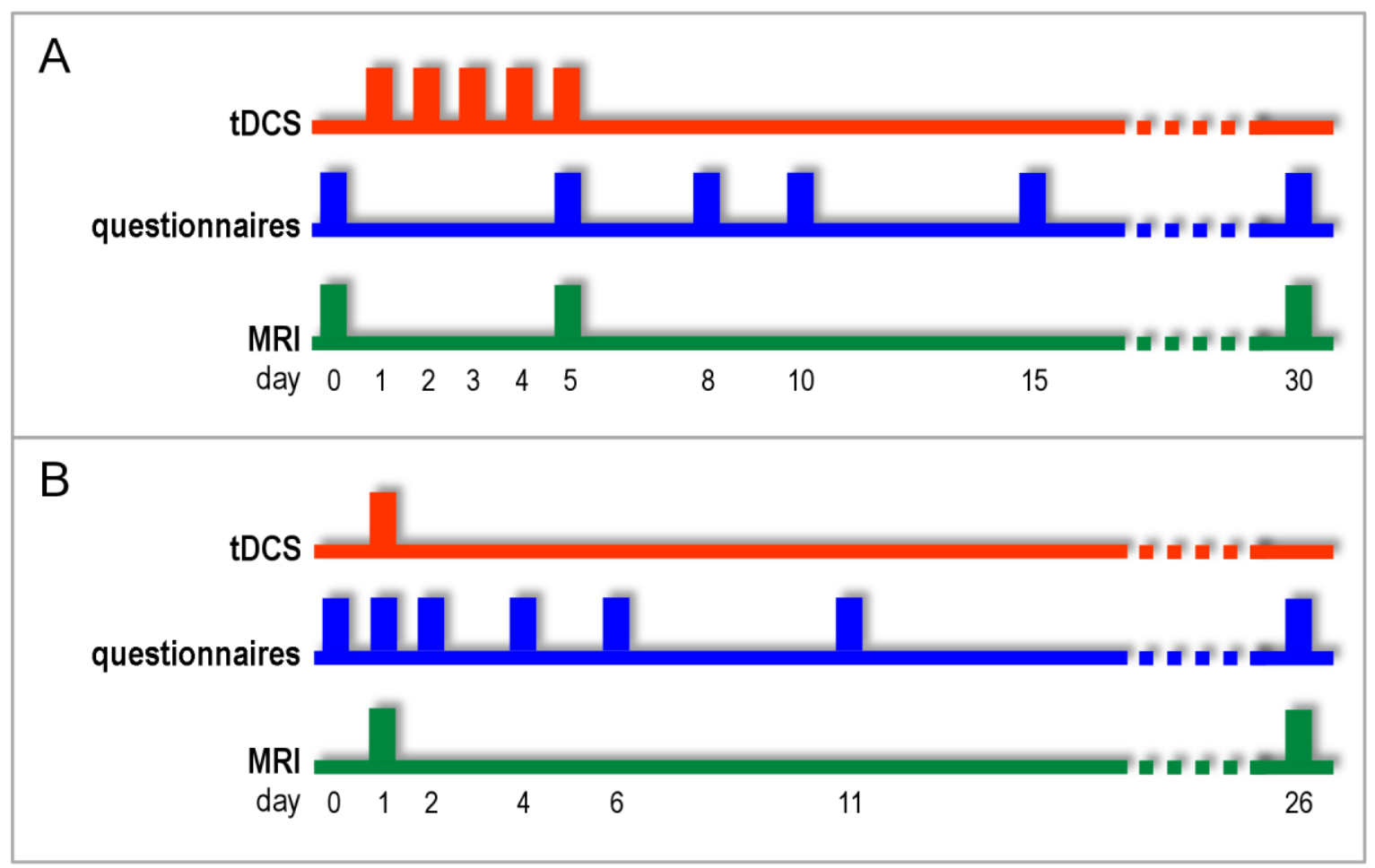

Figure 9. Experimental protocol. Patients underwent the protocol twice, once for anodal tDCS and once for sham stimulation, separated by at least 2 weeks. On day 0 , baseline fatigue scores and confirmation of inclusion criteria regarding lesions and depression were obtained. A) Protocol 1: patients received stimulation from days 1 to 5 . After stimulation on day 5 , patients answered the questionnaires and underwent MRI examination. Follow-up sessions took place on days 8, 10, 15 and 30. Day 30 included another MRI session. B) Protocol 2: patients received stimulation and underwent an MRI session on day 1. Follow-up sessions were performed on days $2,4,6,11$ and 26 .

\subsubsection{Neuron-specific enolase (NSE)}

In protocol 1, we analysed serum-NSE levels to ensure safety of the stimulation. Serum-NSE is a sensitive marker of neuronal-damage (Steinhoff et al., 1999). Blood samples were taken 
on stimulation days 1 and 5 (Figure. 9A) before, immediately after and one hour after tDCS. We performed an interim analysis of serum-NSE levels to confirm safety of the procedure to continue with the study.

\subsubsection{Analysis of the questionnaires}

Statistical analysis was performed using SPSS, version 21.0. The scores of the FSS and MSFSS were calculated by averaging all items, whereas for the MFIS, the BDI and the HADS, the total score was determined by summing the individual items. Pearson's correlation was calculated pairwise between baseline scores of the FSS, MSFSS, MFIS and subscales, and fatigue VAS.

Because data from the questionnaires is ordinal, non-parametric Friedman tests were performed to test for changes during each block with regard to the fatigue scales and VAS, the BDI, HADS-D and HADS-A, separately for sham and real stimulation. When significant effects were found, pairwise comparisons between baseline and each follow-up measurement were performed by using Wilcoxon signed-rank test for related samples. To analyse the effect of anodal tDCS, the changes in the scores were computed by normalising each day to baseline, and then compared within each day between sham and real stimulation with a Wilcoxon signed-rank test.

Wilcoxon Mann-Whitney tests were used to compare the normalized scores with regard to an order effect (sham first $\mathrm{N}=6$, tDCS first $\mathrm{N}=7$ ), for days 5 and 30 of each block, only for protocol 1 due to the small sample size of protocol 2.

Based on the reported change in perceived fatigue, the patients were classified as responders, if real stimulation resulted in stronger improvement than sham and non-responders otherwise. The analyses were repeated for responders separately. A response index was calculated by subtracting the change in perceived fatigue after tDCS from the change in perceived fatigue after sham. Therefore, the higher the response index the better was the reaction to real stimulation. Exploratory analyses were run to compare responders and nonresponders regarding fatigue, depression and MRI measures.

Given the exploratory nature of the study, we discuss those results, which are significant at a level of 0.05 , without correction of the post-hoc tests for multiple comparisons. 


\subsubsection{MRI analysis}

Gadolinium-enhanced T1-weighted images were inspected visually by an experienced investigator for the presence of active lesions. One patient (protocol 1) was excluded from MRI analysis due to poor image quality. Lesion masks were obtained using a k-nearest neighbour algorithm (Anbeek et al., 2004). In short, this algorithm compares the brain voxels of a newly presented dataset to a collection of manually labelled examples. The features included T2-FLAIR and T1-weighted signal intensity; normalized spatial coordinates, $\mathrm{x}, \mathrm{y}$ and z; and tissue type priors describing the suspected tissue class (i.e. CSF, GM or WM) to which the voxel would have belonged before the lesion developed (Steenwijk et al., 2013). The resulting lesion masks were manually corrected with assistance of an experienced investigator before any subsequent analysis steps.

All other analysis steps were performed using FSL (Jenkinson et al., 2012). T2-FLAIR images were registered to the T1 images using FLIRT (Jenkinson et al., 2002) (12 DOF). The same transformation was applied to the lesion masks using nearest neighbour interpolation, to avoid expansion of the masks caused by linear interpolation. The lesion masks in T1 space were visually inspected individually and small corrections were performed when necessary. The presence of lesions in T1-weighted images (Figure 10A) has been shown to influence registration to standard space, particularly when using non-linear algorithms, which then biases the calculation of brain tissue volumes, in particular that of GM (Battaglini et al., 2012). Therefore, lesion masks were used for lesion-filling of T1 images (Figure 10B) using the algorithm implemented in FSL (Battaglini et al., 2012). In the case of big lesions, the filled T1 image still showed some hypointense voxels within the lesions (Figure 10C). Therefore, an eroded version of the lesion mask was created, and used to perform a second round of lesion filling (Figure 10D). Lesion-filled T1 images were registered to the MNI standard space, first using FLIRT, afterwards improved with non-linear transformation with FNIRT (Andersson et al., 2007). The calculated transformations were applied to the lesion masks and the lesions volumes (LV) were calculated in standard space. Frontal, temporal, parietal and occipital gray and white matter masks were created based on MNI Structural Atlas available in FSL and LV was calculated separately for each of these regions. Total gray matter, white matter and whole brain volumes normalized to head size were calculated with SIENAX (Smith et al., 2002). 

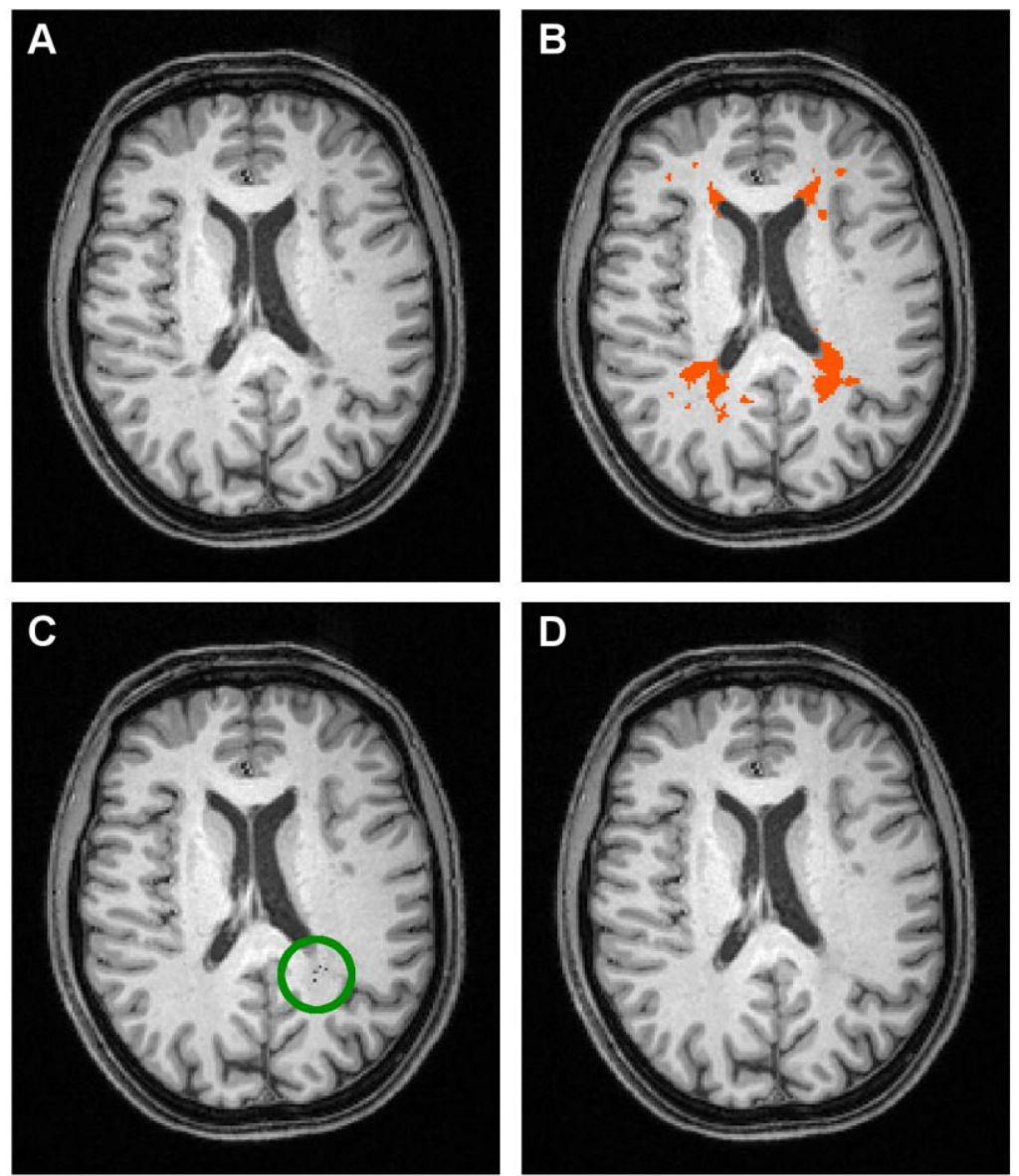

Figure 10. Lesion-filling of T1-weighted images. A) Black holes in T1 images can affect the registration to standard space. B) Lesion masks in T1-space were used to perform lesion-filling. C) After the lesion-filling, some hypointense voxels remain (green circle). D) After re-running the lesion-filling algorithm with an eroded version of the lesion mask, the intensities are corrected. 


\subsection{Results}

\subsubsection{Stimulation sensation}

All patients tolerated the stimulation well, no treatment sessions were interrupted and neither pain under the electrodes nor serious side-effects were reported. One patient had moderate headache after both sham and real stimulation (protocol 1). Four patients reported stronger skin sensations during real (weak to moderate tingling) than during sham (no sensation) (protocol 1). Out of these, three patients answered with yes, when asked explicitly whether they had been able to distinguish between the two stimulation conditions: one patient stated stronger tingling in the first block (real) than the second (sham) whereas the other described the difference solely in terms of changes in fatigue, as being less tired in the first block (real) and normally tired in the second (sham). The third patient reported being able to distinguish between stimulation conditions, by feeling stronger tingling during sham (first block) than during real tDCS (second block) (protocol 1). Thus, overall most of the patients were not able to distinguish between anodal tDCS and sham stimulation for either protocol.

\subsubsection{Neuron-specific enolase}

Two patients did not agree with the blood taking procedure. For the remaining 11 patients, baseline mean NSE values were $11.81 \pm 1.19 \mu \mathrm{g} / \mathrm{L}$ before the first tDCS session. Paired samples $t$-tests revealed no significant differences ( $\mathrm{p}>0.05)$ in NSE levels immediately after or 1 hour after stimulation compared to baseline (Figure 11).

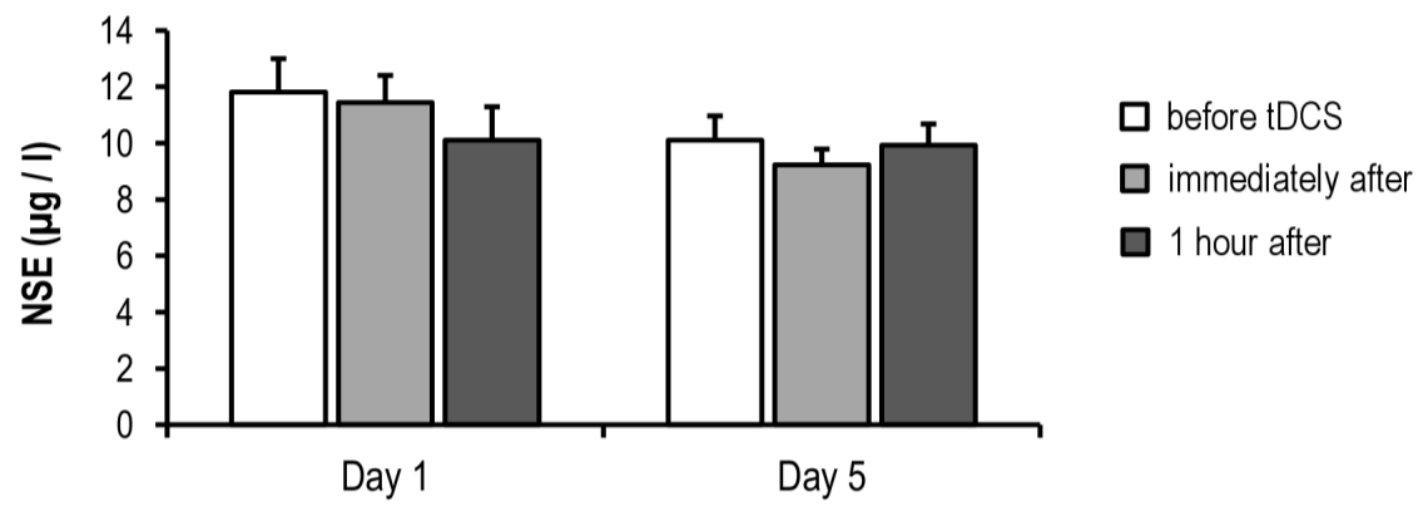

Figure 11. NSE values before and after stimulation, in protocol 1 (11 patients). Paired samples t-tests revealed no significant differences $(p>0.05)$ in NSE levels on Day 1 and Day 5. 


\subsubsection{Correlation of fatigue scales at baseline}

Baseline scores (day o of the first block) of the fatigue scales, BDI, HADS-D, disease duration, EDSS and normalized brain volumes for both protocols are presented in Table 6 .

Table 6. Demographic, clinical and structural MRI characteristics of all patients.

\begin{tabular}{|c|c|c|c|c|}
\hline & \multicolumn{2}{|c|}{ Protocol 1} & \multicolumn{2}{|c|}{ Protocol 2} \\
\hline & Mean & SD & Mean & SD \\
\hline Age (yrs) & 46.9 & 6.8 & 44.4 & 11.2 \\
\hline $\operatorname{EDSS}^{\mathrm{a}}(1-10)$ & 3.5 & 4.0 & 3.25 & 1.64 \\
\hline Disease duration (yrs) & 9.0 & 5.4 & 9.8 & 5.4 \\
\hline $\mathrm{FSS}^{\mathrm{a}}(1-7)$ & 5.67 & 2.47 & 5.39 & 2 \\
\hline $\operatorname{MSFSS}^{\mathrm{a}}(1-7)$ & 5.00 & 3.33 & 4.33 & 2.17 \\
\hline MFIS $^{a}(0-84)$ & 47 & 31 & 52 & 43 \\
\hline $\mathrm{BDI}^{\mathrm{a}}(0-63)$ & 4 & 11 & 5.5 & 13 \\
\hline HADS depression $^{a}(0-21)$ & 2 & 6 & 4 & 8 \\
\hline HADS anxiety ${ }^{\mathrm{a}}(0-21)$ & 4 & 8 & 4 & 5 \\
\hline NBV (mL) & 1491 & 77 & 1482 & 87 \\
\hline NWMV (mL) & 705 & 38 & 706 & 22 \\
\hline NGMV (mL) & 786 & 50 & 775 & 68 \\
\hline
\end{tabular}

For all patients (protocol 1 and protocol 2), at baseline, the FSS scores tendentiously correlated with the global MFIS but not significantly ( $N=21$, Pearson's $r=0.398 ; \mathrm{p}=0.078$ ) and the correlation was stronger with the subscale MFISphy $(\mathrm{N}=21$, Pearson's $r=0.576$; $\mathrm{p}=0.039)$, but not with the subscales MFISpsych $(\mathrm{N}=21$, Pearson's $r=0.219 ; \mathrm{p}=0.341)$ and MFIScog $(\mathrm{N}=21$, Pearson's $r=0.250 ; \mathrm{p}=0.274)$. The MSFSS did not correlate with the FSS ( $N=21$, Pearson's $r=-0.210 ; \mathrm{p}=0.361)$, nor with the MFIS ( $\mathrm{N}=21$, Pearson's $r=-0.047$; $\mathrm{p}=0.839$ ) or any of the subscales. In addition, for protocol 2, the VAS did not correlate with any other fatigue scale. The scores of the fatigue scales did not correlate significantly with disease duration, age or EDSS scores.

\subsubsection{Subjective changes of fatigue}

\section{Protocol 1}

Regarding the fatigue scales (Figure 12), there was a significant effect of time in the real stimulation block for global MFIS $\left(\chi^{2}{ }_{(5)}=14.484 ; \mathrm{p}=0.013\right)$ and MFISpsych scores $\left(X^{2}(5)=13.931 ; p=0.016\right)$, as well as on the MFIScog scores for both, the real $\left(\chi^{2}(5)=13.794\right.$; $\mathrm{p}=0.017)$ and sham $\left(\chi^{2}(5)=11.754 ; \mathrm{p}=0.038\right)$ blocks, the respective scores decreased as 
compared to baseline. However, according to the results of the post-hoc Wilcoxon signedrank tests, the changes did not differ significantly between real and sham stimulation. All other Friedman tests on the fatigue scales showed non-significant results (Table 7).

Table 7. Results of the Friedman tests for fatigue levels after anodal tDCS and sham stimulation.

\begin{tabular}{|c|c|c|c|c|c|}
\hline \multirow[b]{2}{*}{ Scale } & \multirow[b]{2}{*}{ stimulation } & \multicolumn{2}{|c|}{ Protocol $1(\mathrm{~N}=13)$} & \multicolumn{2}{|c|}{ Protocol $2(\mathrm{~N}=8)$} \\
\hline & & $x^{2}(5)$ & $\mathbf{p}$ & $x^{2}(6)$ & $\mathbf{p}$ \\
\hline \multirow[t]{2}{*}{ perceived fatigue } & sham & 10.325 & 0.067 & 3.700 & 0.593 \\
\hline & real & 17.079 & 0.004 & 6.385 & 0.271 \\
\hline \multirow[t]{2}{*}{ FSS } & sham & 2.471 & 0.781 & 3.317 & 0.768 \\
\hline & real & 3.023 & 0.696 & 2.501 & 0.868 \\
\hline \multirow[t]{2}{*}{ MSFSS } & sham & 2.693 & 0.747 & 4.443 & 0.617 \\
\hline & real & 2.430 & 0.787 & 6.219 & 0.399 \\
\hline \multirow[t]{2}{*}{ MFIS } & sham & 6.678 & 0.246 & 8.718 & 0.190 \\
\hline & real & 14.484 & 0.013 & 11.610 & 0.071 \\
\hline \multirow[t]{2}{*}{ MFISphy } & sham & 3.255 & 0.661 & 3.825 & 0.700 \\
\hline & real & 10.047 & 0.074 & 12.364 & 0.054 \\
\hline \multirow[t]{2}{*}{ MFISpsych } & sham & 4.740 & 0.448 & 13.320 & 0.038 \\
\hline & real & 13.931 & 0.016 & 5.188 & 0.520 \\
\hline \multirow[t]{2}{*}{ MFIScog } & sham & 11.754 & 0.038 & 11.104 & 0.085 \\
\hline & real & 13.794 & 0.017 & 9.609 & 0.142 \\
\hline \multirow[t]{2}{*}{ VAS } & sham & - & - & 3.426 & 0.754 \\
\hline & real & - & - & 16.294 & 0.012 \\
\hline
\end{tabular}

Regarding the change in perceived fatigue, the results of the Friedman tests show a significant decrease during the real stimulation block $\left(\chi^{2}(5)=17.079 ; p=0.004\right)$, but close to significance also during the sham block $\left(\chi^{2}(5)=10.325 ; p=0.067\right)$ (Table 7). Post-hoc analysis with the Wilcoxon signed-rank tests showed that the decrease was significant versus baseline on days 5 to 10 and close to significance on days 15 and 30 under real stimulation conditions, but the changes were not significantly different from sham on any day (Figure 13A). 
A

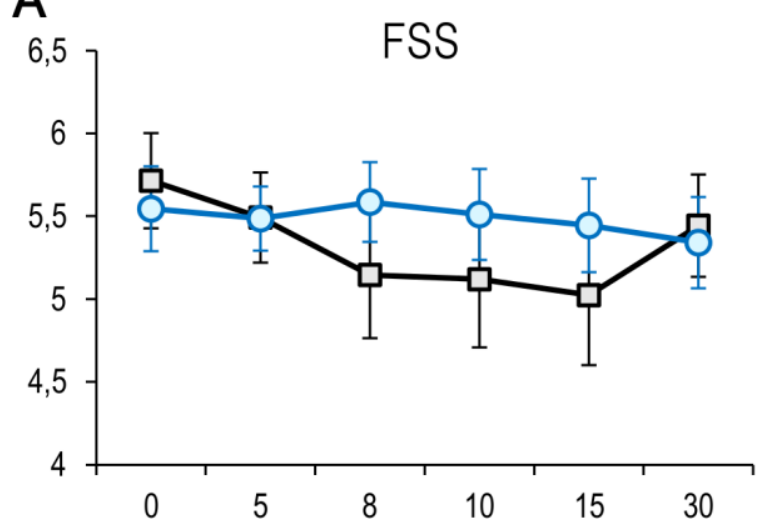

\section{C}

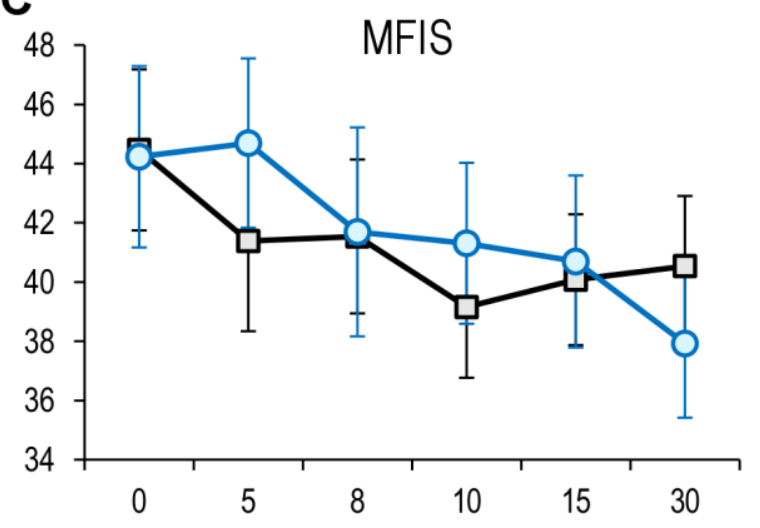

E

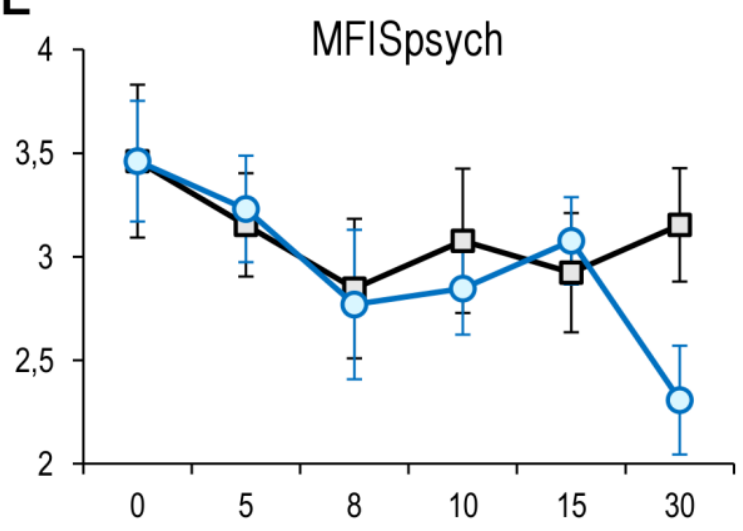

Day
B

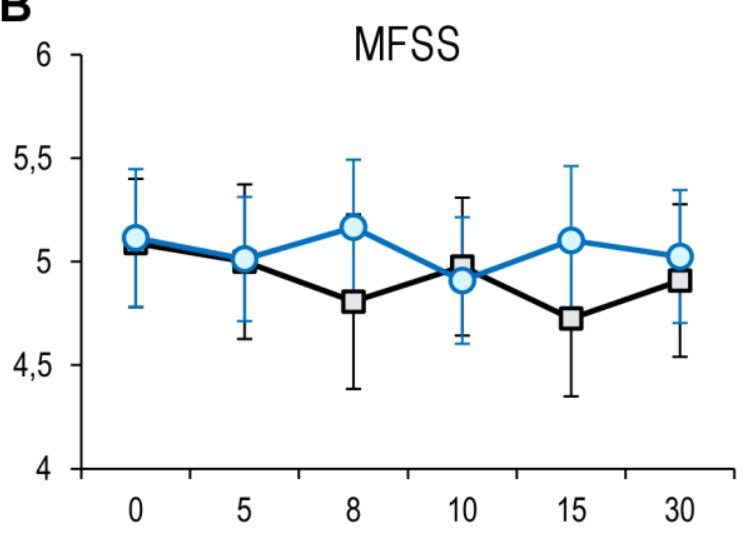

D

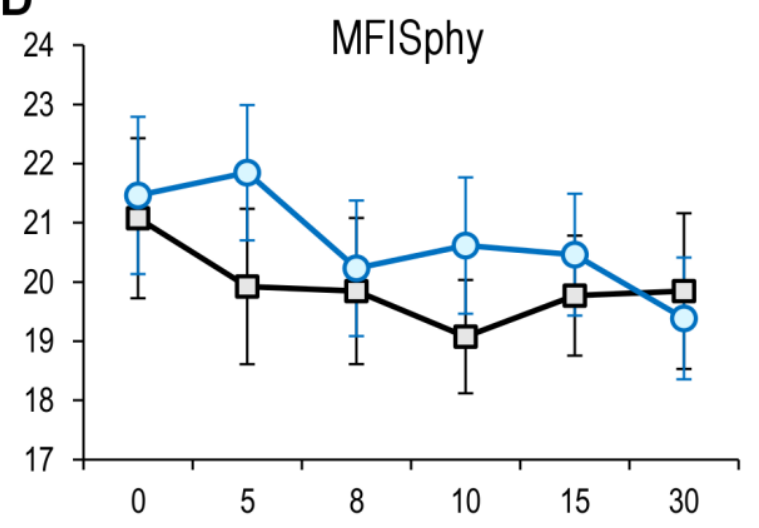

$\mathbf{F}$

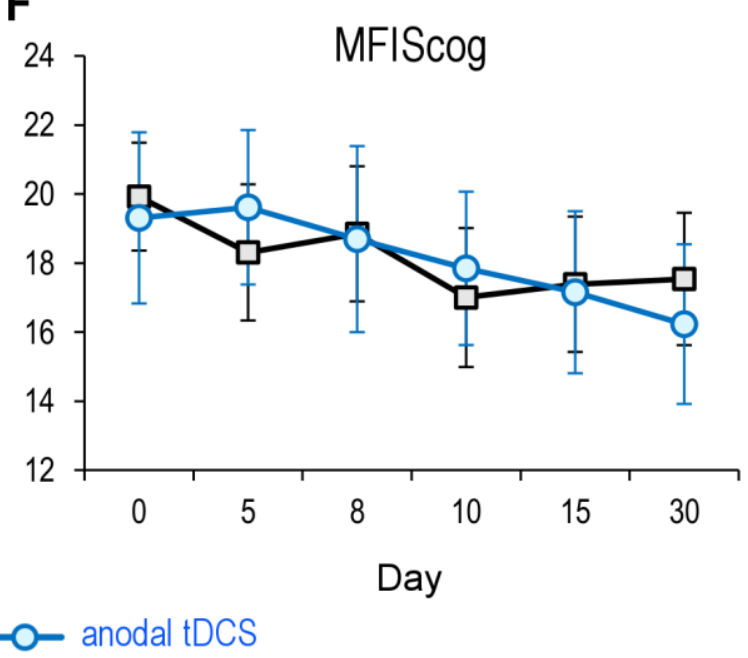

Figure 12. Fatigue scales (protocol 1). A) FSS. B) MSFSS. C) Compared to baseline, the scores of the MFIS and E) MFISpsych decreased significantly after real stimulation block $(p=0.013 ; p=0.016$, respectively). D) MFISphy. F) The scores of the MFIScog decreased significantly after both the real $(p=0.017)$ and sham stimulation $(p=0.038)$. There were no significant differences between the decrease after real and sham stimulation. Error bars represent SEMs. 
A

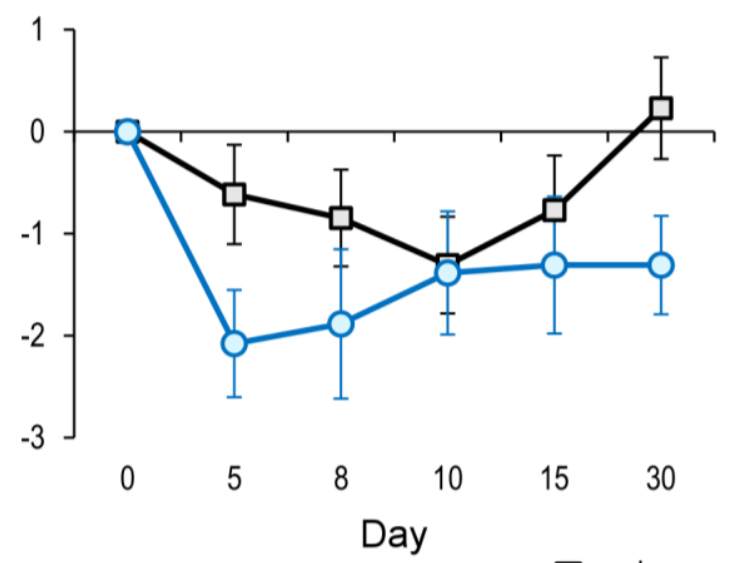

Protocol $1(\mathrm{~N}=13)$ $\longrightarrow$ - sham $\longrightarrow$ - anodal tDCS
B Protocol $2(\mathrm{~N}=8)$

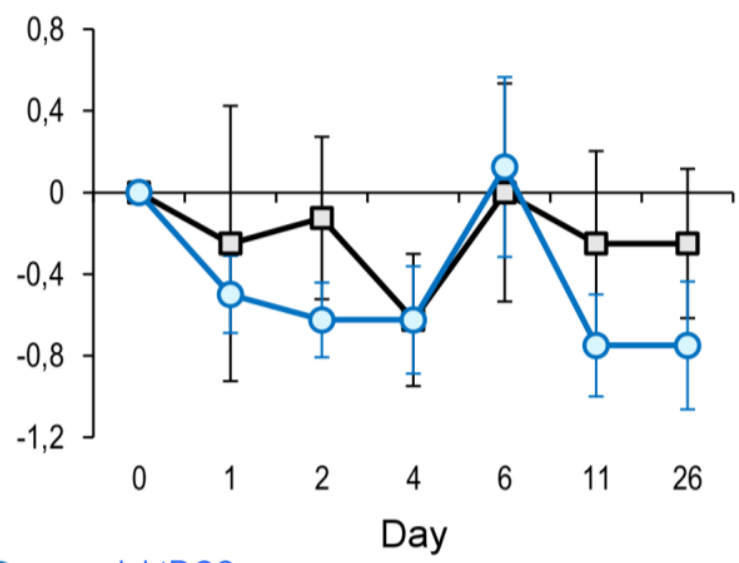

Figure 13. Changes in perceived fatigue after real and sham stimulation. A) Protocol 1: there was a significant decrease in the anodal tDCS block $(p=0.004)$, but not significantly different from the changes in the sham block. B) Protocol 2: changes in perceived fatigue were not significant. Error bars represent SEMs.

Order effects were found for the global MFIS scores (U=5.0; $\mathrm{Z}=-2.286 ; \mathrm{p}=0.022)$ and the subscales MFISphy ( $U=5.0 ; Z=-2.292 ; p=0.022)$ and MFISpsych $(U=3.5 ; Z=-2.579 ; p=0.010)$ on day 30 after anodal tDCS; and after sham stimulation on day 5 for the MFISpsych (U=6.0; $\mathrm{Z}=-2.234 ; \mathrm{p}=0.026$ ), arising from greater reduction in the scores during the first block than during the second block (Figure 14).

\section{Protocol 2}

There were no significant changes in perceived fatigue (Figure 13B). The VAS values for fatigue (Figure 15) decreased significantly after real stimulation $\left(\chi^{2}(6)=16.294 ; p=0.012\right)$, and post-hoc Wilcoxon signed-rank tests showed that scores were significantly different from baseline on days $2(\mathrm{Z}=-2.388 ; \mathrm{p}=0.017), 11(\mathrm{Z}=-2.371 ; \mathrm{p}=0.018)$ and $26(\mathrm{Z}=-2.527 ; \mathrm{p}=0.012)$. However, the changes were not significantly different from sham on any day (Mann Whitney U tests). Although the scores of the MFIS and MFISphy tendentiously decreased, the only significant result was observed in the MFISpsych in the sham stimulation block (Figure 16). This was caused by the significantly increased scores on day $1(\mathrm{Z}=-2.00 ; \mathrm{p}=0.046)$. Other Friedman tests on the fatigue scales showed non-significant results (Table 7). 

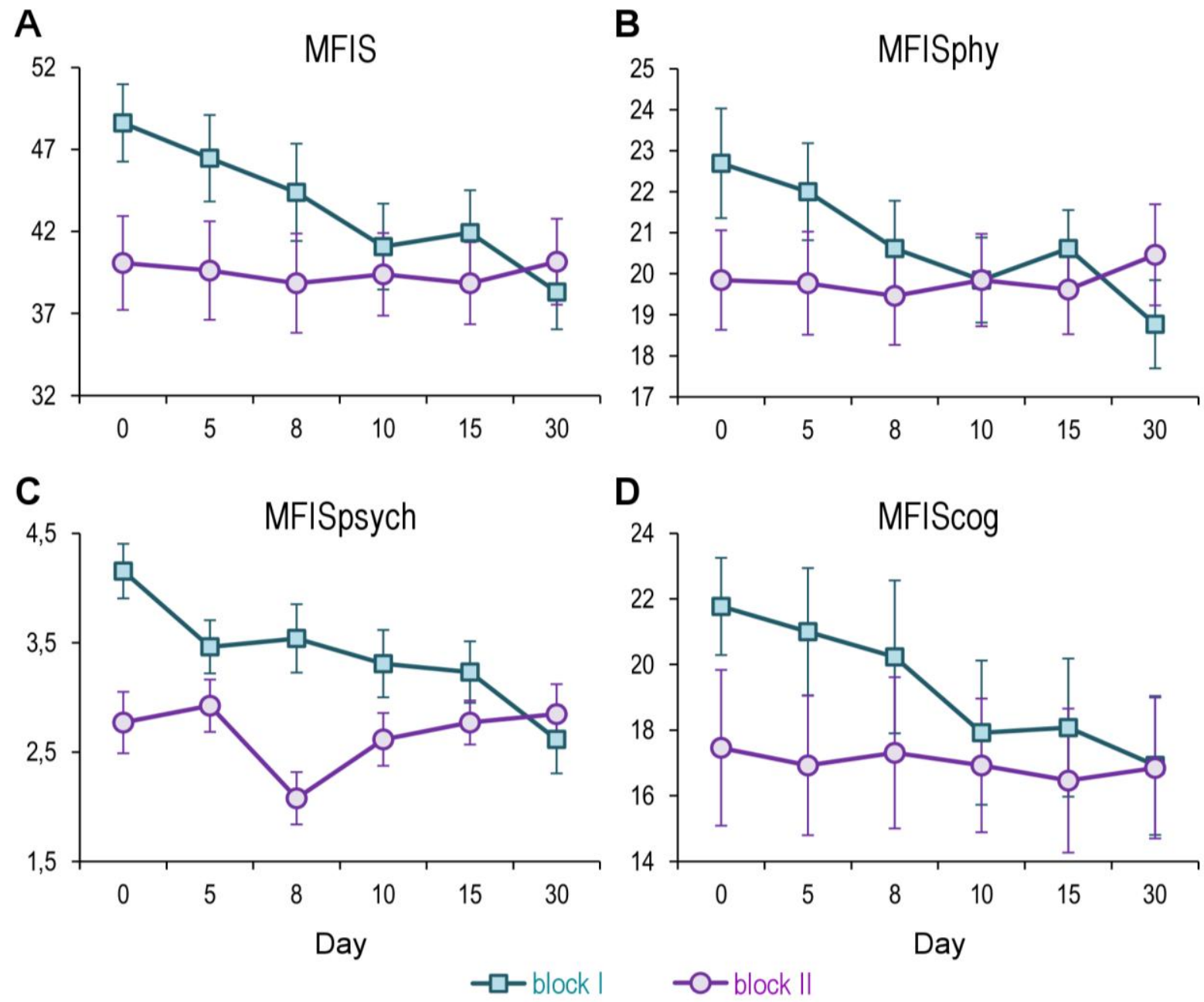

Figure 14. Order effects in the MFIS (protocol 1). The scores of the MFIS and the subscales decreased more in the first block than in the second block. A) Order effects were significant for the MFIS $(p=0.022), B)$. MFISphy $(p=0.022)$ and C) MFISpsych $(p=0.010)$ on day 30 after anodal tDCS and after sham stimulation on day 5 for the MFISpsych $(p=0.026)$. D). MFIScog. Error bars represent SEMs.

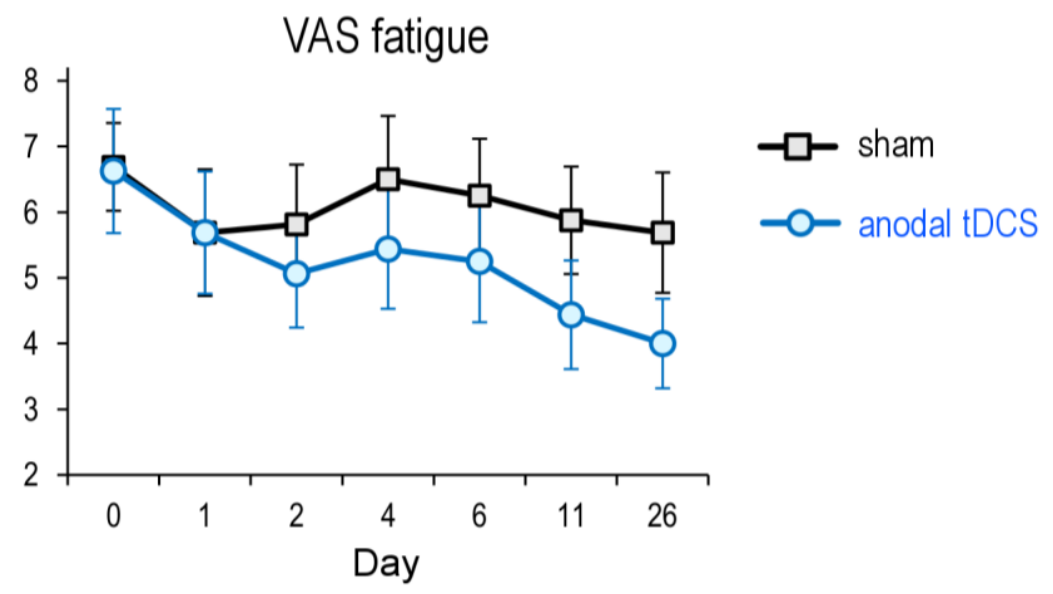

Figure 15. Changes in the VAS for fatigue (protocol 2). The VAS decreased significantly after real stimulation $(p=0.012)$. Post-hoc Wilcoxon signed-rank tests showed that scores were different from baseline on days $2(p=0.017), 11(p=0.018)$ and $26(p=0.012)$. The changes were not significantly different from sham on any day. Error bars represent SEMs. 

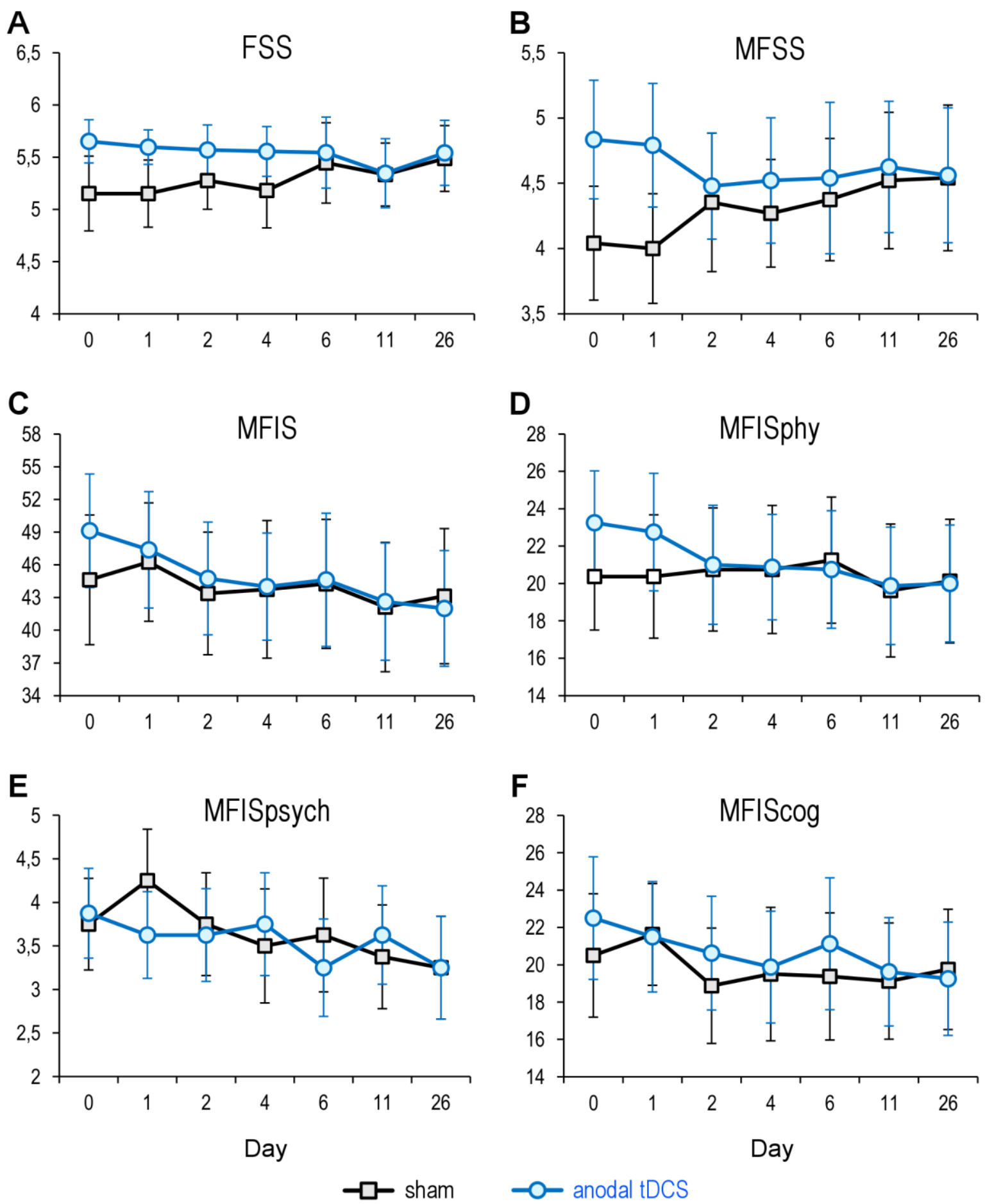

Figure 16. Fatigue scales (protocol 2). A) FSS. B) MSFSS. C) There was a non-significant decrease in the scores of the MFIS $(p=0.071)$ and $D)$ MFISphy (0.054) after real stimulation. E) The MFISpsych scores changed significantly after sham stimulation $(p=0.038)$ due to the increased scores on day 1. F) MFIScog. Error bars represent SEMs. 
The order effects on the fatigue scales were significant on the MSFSS right after stimulation (day 1) $(U=13.0 ; Z=-2.057 ; p=0.040)$ as they increased in the group receiving sham stimulation in first block and decreased in the group receiving real stimulation in the second block. No significant order effects on days 2 or 27 were found for any other fatigue scale.

\subsubsection{Variation of depression and anxiety levels}

\section{Protocol 1}

The Friedman test showed a significant effect of time in BDI scores in the sham block $\left(\chi^{2}(5)=18.711 ; p=0.002\right)$ and in HADS-A scores in both sham $\left(\chi^{2}{ }_{(5)}=25.910 ; p<0.001\right)$ and real $\left(\chi^{2}(5)=12.772 ; p=0.026\right)$ blocks (Table 8). Post-hoc analysis with Wilcoxon signed-rank tests showed that BDI scores decreased compared to baseline on days 5 to 15 , HADS anxiety scores were lower than baseline on all days in the sham block, but only on days 8 ( $p=0.009), 15$ $(\mathrm{p}=0.030)$ and $30(\mathrm{p}=0.026)$ in the real stimulation block. Post-hoc Wilcoxon signed-rank tests of the normalized BDI and HADS-A scores revealed no significant differences between sham and real tDCS on any day (Figure 17).

Order effects were found on HADS-D scores for days $5(\mathrm{U}=7.0 ; \mathrm{Z}=-2.051 ; \mathrm{p}=0.040)$ and 30 $(U=6.5 ; Z=-2.162 ; p=0.031)$ after sham stimulation, on BDI scores for day $30(U=6.0 ; Z=-$ 2.198; $\mathrm{p}=0.028$ ) after sham stimulation and for HADS-A scores for day 30 after real stimulation ( $\mathrm{U}=7.0 ; \mathrm{Z}=-2.037 ; \mathrm{p}=0.042)$. In all of these cases, scores decreased more in the first than in the second block (Figure 18).

Table 8. Results of the Friedman tests for depression and anxiety levels after anodal tDCS and sham stimulation.

\begin{tabular}{llcccc}
\hline & & \multicolumn{2}{c}{ Protocol 1 $(\mathbf{N}=\mathbf{1 3})$} & \multicolumn{2}{c}{ Protocol $\mathbf{2}(\mathbf{N}=8)$} \\
\hline scale & stimulation & $\mathbf{X}^{\mathbf{2}}(\mathbf{6})$ & $\mathbf{p}$ & $\mathbf{X}^{\mathbf{2}}(\mathbf{6})$ & $\mathbf{p}$ \\
\hline BDI & sham & $\mathbf{1 8 . 7 1 1}$ & $\mathbf{0 . 0 0 2}$ & 8.193 & 0.224 \\
& real & 6.173 & 0.290 & 2.934 & 0.817 \\
HADS-A & sham & $\mathbf{2 5 . 9 1 0}$ & $<\mathbf{0 . 0 0 1}$ & 2.289 & 0.891 \\
& real & $\mathbf{1 2 . 7 7 2}$ & $\mathbf{0 . 0 2 6}$ & 8.771 & 0.187 \\
HADS-D & sham & 7.431 & 0.191 & 5.662 & 0.462 \\
& real & 2.347 & 0.799 & 2.274 & 0.893 \\
\hline
\end{tabular}


Protocol 1

A

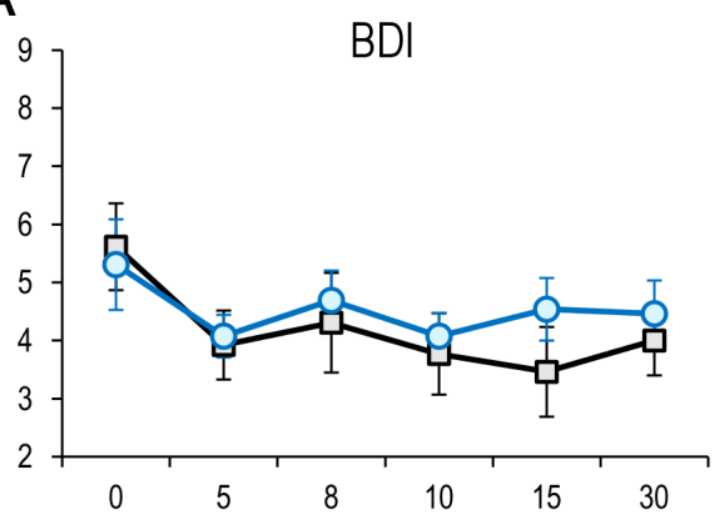

C

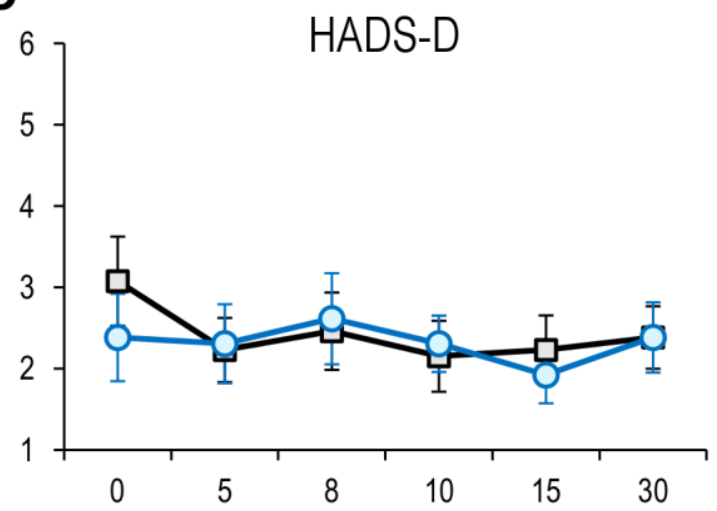

E

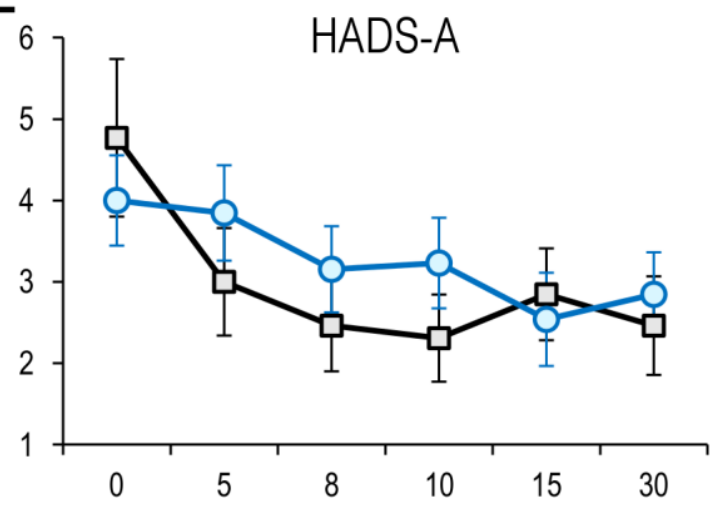

Day
Protocol 2

B

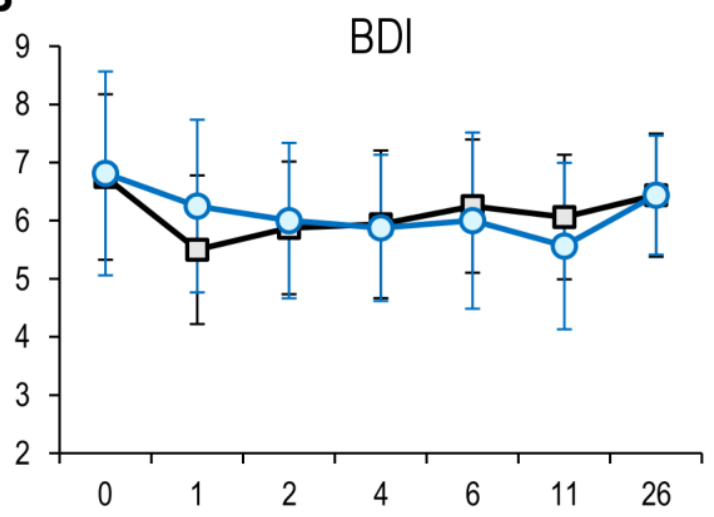

D

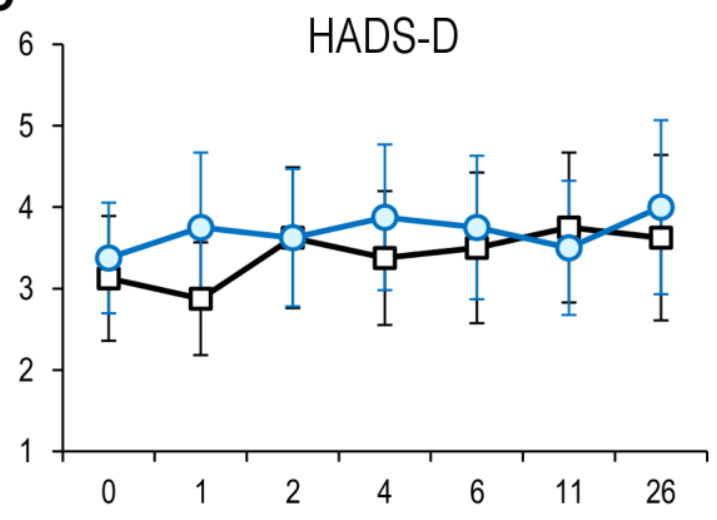

$\mathbf{F}$

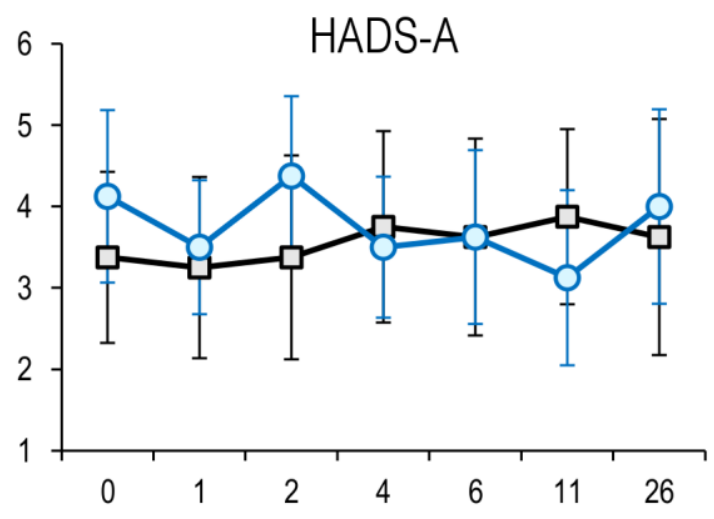

Day

Figure 17. Changes in the BDI, HADS-D and HADS-A scores, on protocol $1(\mathrm{~N}=13)$ and protocol $2(\mathrm{~N}=8)$. A) BDI scores changed significantly after sham stimulation (0.002), on protocol 1. C) HADS-D. E) The HADS-A scores decreased significantly after anodal tDCS $(p=0.026)$ and sham stimulation $(p<0.001)$, on protocol 1. There were no significant differences between sham and real stimulation. No significant changes occurred on protocol 2 (B, D, F). Error bars represent SEMs. 

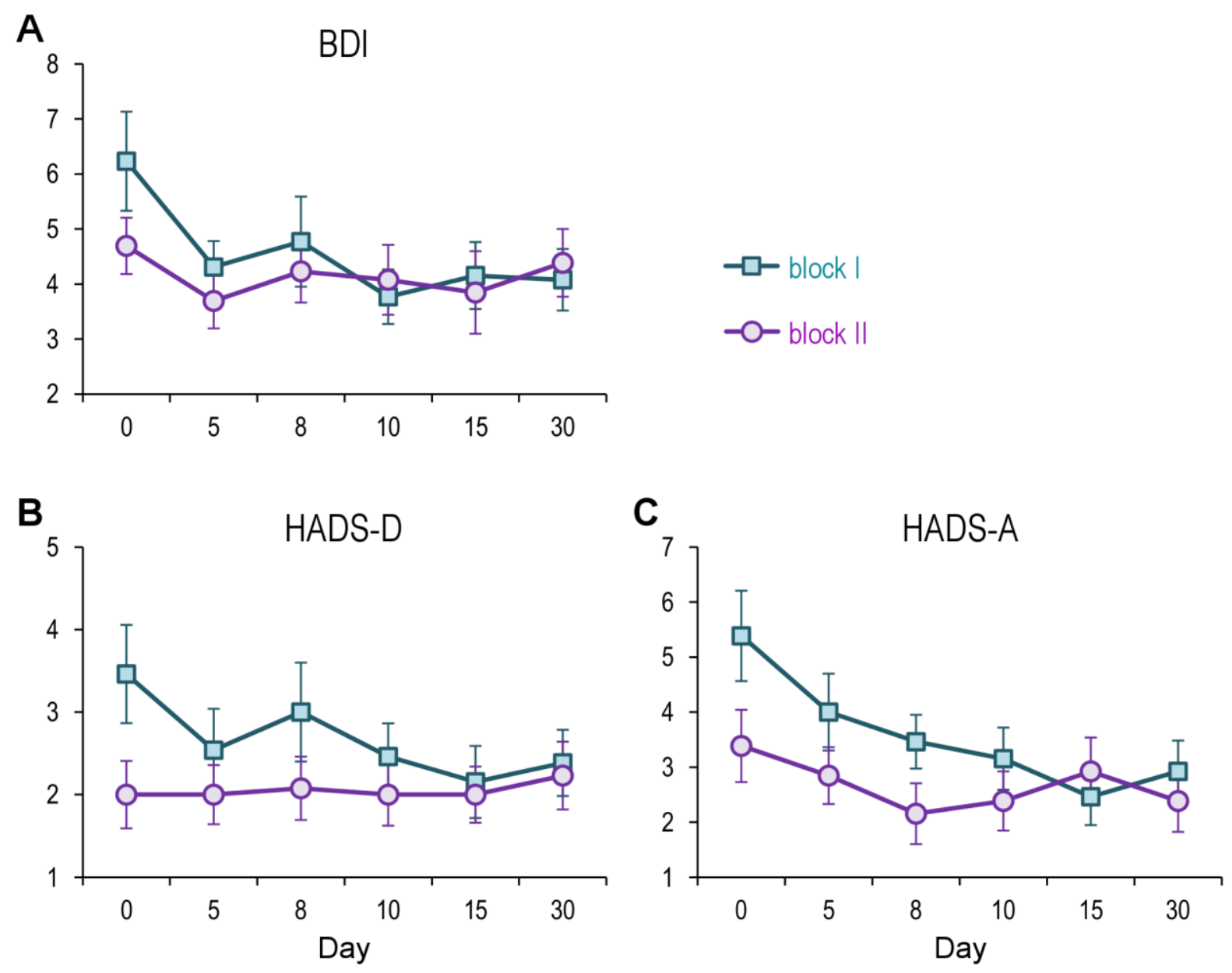

Figure 18. Order effects in the BDI, HADS-D and HADS-A (protocol 1). The depression and anxiety scores decreased more in the first block than in the second block. A) Order effects were significant after sham stimulation on BDI scores on day $30(p=0.028)$ and $B)$ on the HADS-D scores on days $5(p=0.040)$ and $30(p=0.031)$. C) On day 30 after real stimulation, order effects were significant for HADS-A $(p=0.042)$. Error bars represent SEMs.

\section{Protocol 2}

No significant changes in depression and anxiety scores were detected by the Friedman tests for protocol 2 (Table 8).

\subsubsection{Responders vs Non-responders}

\section{Protocol 1}

According to the changes in perceived fatigue, 7 patients were considered as responders and 6 patients as non-responders in protocol 1. Table 9 presents demographic and clinical characteristics separately for responders and non-responders. 
For responders, the Friedman tests (Table 10) revealed a significant change in perceived fatigue in the real $\left(X^{2}(5)=16.546 ; p=0.005\right)$, but not the sham stimulation condition $\left(X^{2}(5)=5.977 ; p=0.308\right)$ (Figure 19). There was also a significant effect of time on the scores of the MFISphy $\left(\chi^{2}(5)=12.651 ; p=0.027\right)$ and MFISpsych $\left(\chi^{2}(5)=14.318 ; p=0.014\right)$ only under real stimulation (Figure 20). Post-hoc Wilcoxon signed-rank tests comparing each day's values with baseline values showed a trend for improvement in perceived fatigue on every day but the last, but not in the MFISphy or MFISpsych. Comparison of normalized values (to baseline) between sham and real stimulation showed a difference in change in perceived fatigue, but not in the MFISphy or MFISpsych scores.

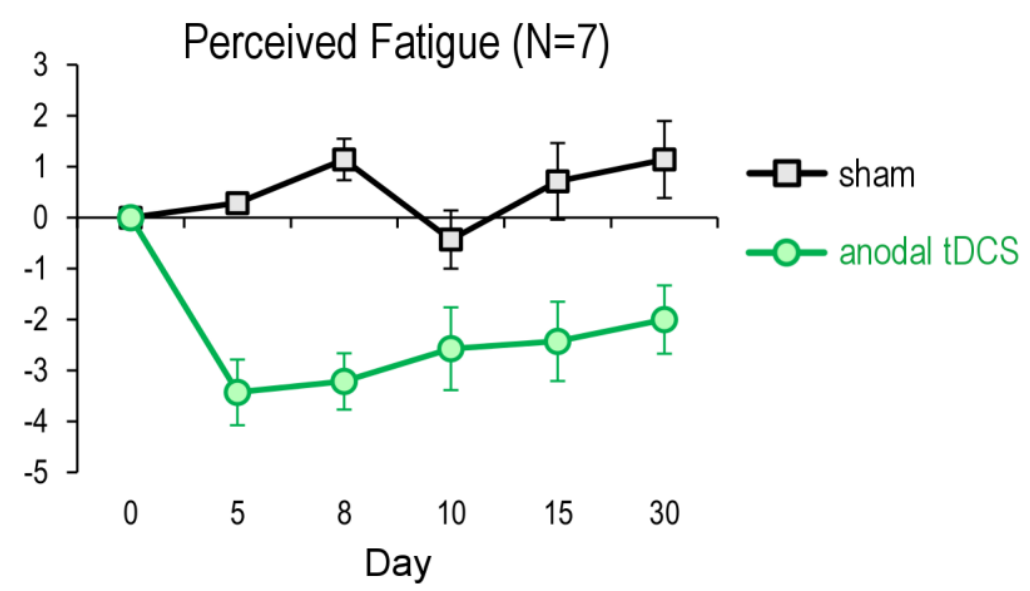

Figure 19. Changes in perceived fatigue in responders. The decrease in perceived fatigue was significant in the real stimulation block $(p=0.005)$. Changes were significantly different between anodal tDCS and sham stimulation. Error bars represent SEMs.

In the subgroup of responders, the scores of the BDI tendentiously decreased, particularly after sham stimulation, but this effect was not significant (Table 11). The decrease in HADS-A scores was significant in the sham block $\left(\chi^{2}(5)=16.777 ; p=0.005\right)$, but not in the real tDCS block $\left(\chi^{2}(5)=10.403 ; p=0.065\right)$ (Figure 21).

Additionally, Mann Whitney U tests were used to compare responders and non-responders with regard to baseline fatigue and depression scores before anodal tDCS and found that responders had higher BDI scores $(U=5.000 ; Z=-2.338 ; p=0.019)$, but did not differ in any other scale, neither in EDSS, age or disease duration (Table 9). Also, the response index did not correlate with baseline fatigue in any scale, age, disability and disease duration. 
A

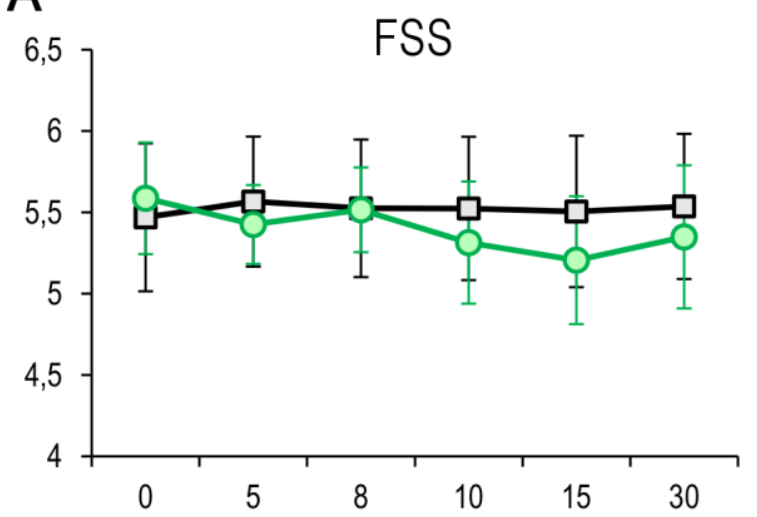

C

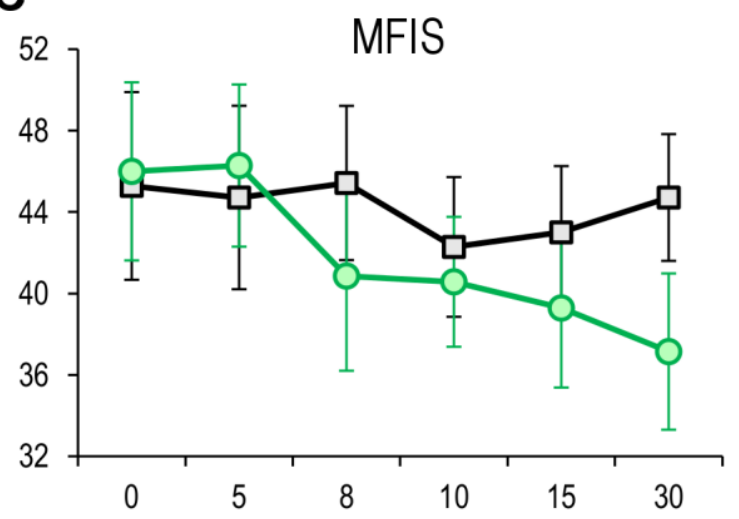

E

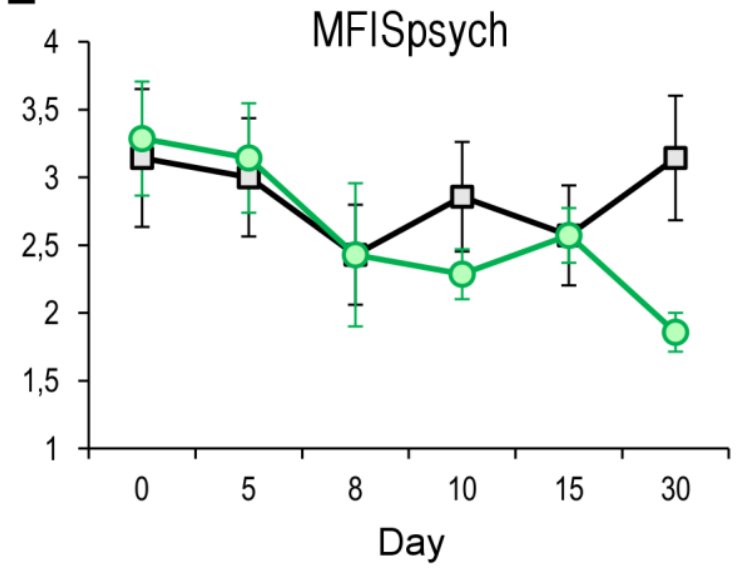

B

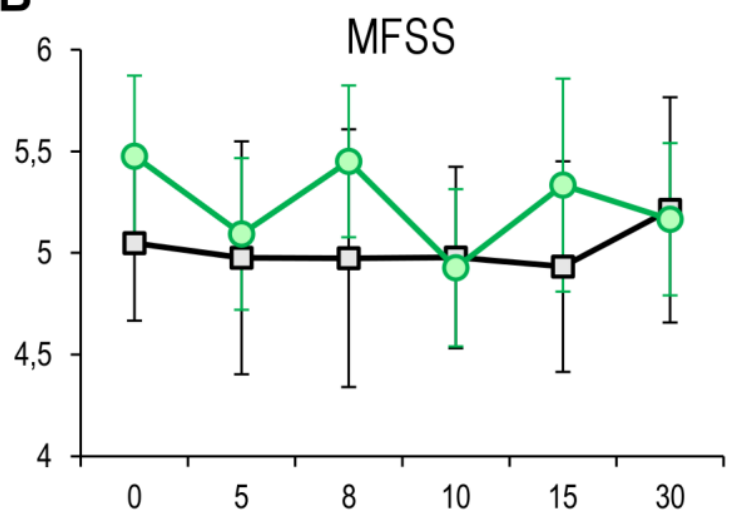

D

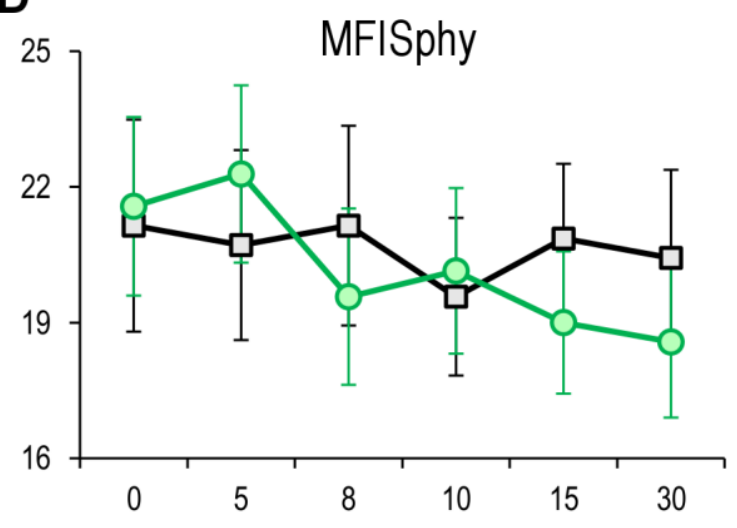

$\mathbf{F}$

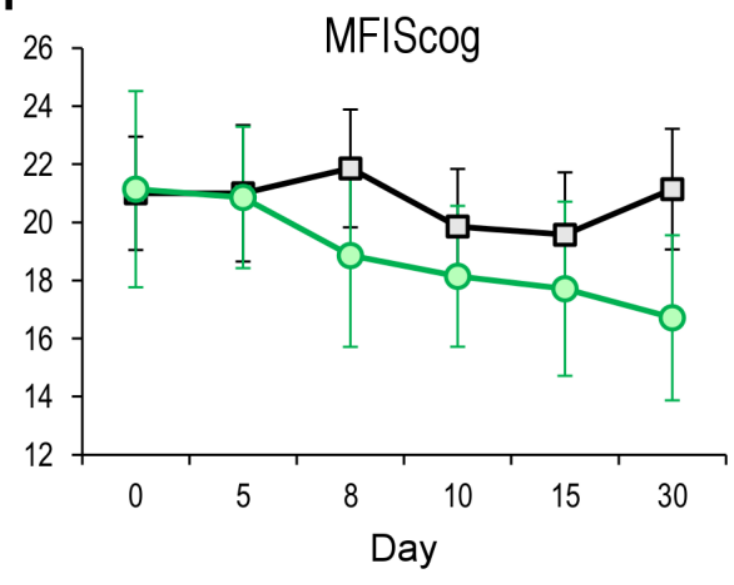

$-\infty$ anodal tDCS

Figure 20. Fatigue scales in responders. A) FSS. B) MSFSS. C) MFIS. D) There was a significant decrease of the MFISphy $(p=0.027)$ and $E)$ MFISpsych $(p=0.014)$ in the real stimulation block. However, changes were not significantly different from baseline or from sham. F) MFIScog. Error bars represent SEMs. 

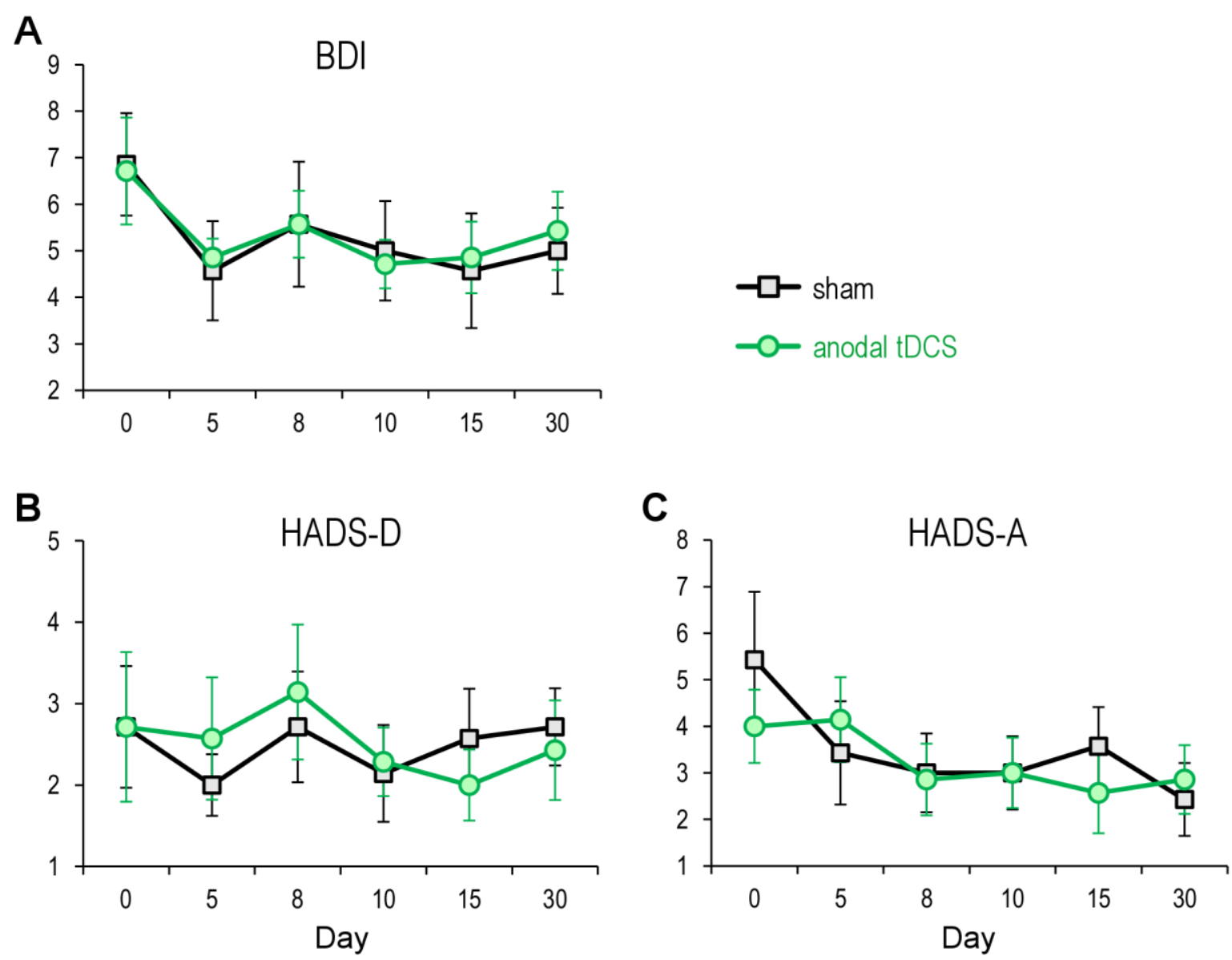

Figure 21. Changes in the BDI, HADS-A and HADS-D, in responders. A) BDI. B) HADS-D. C) The HADS-A scores decreased significantly after sham stimulation $(p=0.005)$. Error bars represent SEMs.

Table 9. Demographic, clinical and structural MRI characteristics of responders and nonresponders

\begin{tabular}{|c|c|c|c|c|}
\hline & \multicolumn{2}{|c|}{ Responders } & \multicolumn{2}{|c|}{ Non-responders } \\
\hline & Mean & SD & Mean & SD \\
\hline Age (yrs) & 46.6 & 5.6 & 47.2 & 8.5 \\
\hline $\operatorname{EDSS}^{\mathrm{a}}(1-10)$ & 3.0 & 2.5 & 3.75 & 4.00 \\
\hline Disease duration (yrs) & 9.4 & 5.4 & 8.5 & 5.9 \\
\hline $\mathrm{FSS}^{\mathrm{a}}(1-7)$ & 5.78 & 2.67 & 5.60 & 2.78 \\
\hline $\operatorname{MSFSS}^{\mathrm{a}}(1-7)$ & 5.00 & 2.67 & 4.67 & 3.17 \\
\hline MFIS $^{a}(0-84)$ & 48 & 28 & 44 & 32 \\
\hline $\mathrm{BDI}^{\mathrm{a}}(0-63)$ & 6 & 9 & 4 & 3 \\
\hline HADS depression ${ }^{\mathrm{a}}(0-21)$ & 2 & 7 & 1.5 & 3 \\
\hline HADS anxiety ${ }^{\mathrm{a}}(0-21)$ & 4 & 5 & 4.5 & 6 \\
\hline NBV (mL) & 1470 & 83 & 1514 & 71 \\
\hline NWMV (mL) & 688 & 54 & 723 & 30 \\
\hline NGMV (mL) & 781 & 40 & 791 & 50 \\
\hline
\end{tabular}

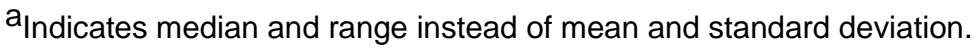


Due to the difference in BDI scores between responders and non-responders, we performed an ordinal logistic regression analysis on the change in perceived fatigue on day 5 (whole patient group) with stimulation and order as factors, and baseline BDI scores before real stimulation as a covariate. The results do not support a significant effect of the BDI scores (Wald $\chi^{2}(1)=1.001 ; p=0.317$ ), neither of order (Wald $\chi^{2}(1)=0.646 ; p=0.421$ ) or stimulation (Wald $\chi^{2}(1)=3.125 ; \mathrm{p}=0.077$ ).

Table 10. Results of the Friedman tests for fatigue levels after anodal tDCS and sham stimulation, in responders.

\begin{tabular}{llcc}
\hline & & \multicolumn{2}{c}{ Responders $(\mathbf{N}=\mathbf{7})$} \\
\hline Scale & Stimulation & $\mathbf{X}^{\mathbf{2}}(\mathbf{5})$ & $\mathbf{p}$ \\
\hline perceived fatigue & sham & 5.977 & 0.308 \\
& real & $\mathbf{1 6 . 5 4 6}$ & $\mathbf{0 . 0 0 5}$ \\
FSS & sham & 2.895 & 0.716 \\
\hline MSFSS & real & 3.333 & 0.649 \\
MFIS & sham & 1.102 & 0.954 \\
& Real & 7.745 & 0.171 \\
MFISphy & sham & 3.369 & 0.643 \\
& real & 10.496 & 0.062 \\
MFISpsych & sham & 1.747 & 0.883 \\
\hline MFIScog & real & $\mathbf{1 2 . 6 5 1}$ & $\mathbf{0 . 0 2 7}$ \\
& sham & 4.253 & 0.514 \\
\hline & real & $\mathbf{1 4 . 3 1 8}$ & $\mathbf{0 . 0 1 4}$ \\
& sham & 6.131 & 0.294 \\
\hline & real & 8.205 & 0.145 \\
\hline
\end{tabular}

Table 11. Results of the Friedman tests for depression and anxiety levels after anodal tDCS and sham stimulation, in responders.

\begin{tabular}{llcc}
\hline & & \multicolumn{2}{c}{ Responders $(\mathbf{N}=\mathbf{7})$} \\
\hline Scale & Stimulation & $\mathbf{X}^{\mathbf{2}} \mathbf{( 5 )}$ & $\mathbf{p}$ \\
\hline BDI & Sham & 10.347 & 0.066 \\
& Real & 6.384 & 0.271 \\
HADS-A & Sham & $\mathbf{1 6 . 7 7 7}$ & $\mathbf{0 . 0 0 5}$ \\
& Real & 10.403 & 0.065 \\
HADS-D & Sham & 8.081 & 0.152 \\
& Real & 0.806 & 0.977 \\
\hline
\end{tabular}




\section{Protocol 2}

Only two patients could be considered as responders according to the changes in perceived fatigue on day 1 . We compared the changes in perceived fatigue with the changes in the VAS for fatigue after anodal tDCS, by calculating the correlation of changes on each day. However, the changes in VAS after anodal tDCS did not correlate with changes in perceived fatigue on any day (Table 12).

Table 12. Correlation between the changes in VAS and changes in perceived fatigue in every

\begin{tabular}{ccc}
\multicolumn{3}{c}{ session after anodal tDCS } \\
\hline & Pearson Correlation (N=8) \\
\hline Session Day & $\boldsymbol{r}$ & $\mathbf{p}$ \\
\hline 2 & -0.227 & 0.507 \\
3 & -0.050 & 0.907 \\
5 & 0.244 & 0.560 \\
7 & 0.190 & 0.653 \\
12 & -0.105 & 0.805 \\
27 & -0.154 & 0.742 \\
\hline
\end{tabular}

\subsubsection{Lesion volumes}

Average normalized GM, WM and whole brain volumes are presented on Table 6 for all patients and on Table 9 for responders and non-responders.

No gadolinium-enhanced lesions were found throughout the study, for either protocol. Therefore, MRI scans at baseline were used for obtaining the lesion masks and for LV calculation (Figure 22). The average LV was $12.4 \pm 3.6 \mathrm{~mL}$ among patients participating in protocol 1 and $18.95 \pm 5.9 \mathrm{~mL}$ in patients participating in protocol 2. LV was compared between responders and non-responders (independent samples t-test, 2 tailed, uncorrected for multiple comparisons). Only LV within the left frontal mask differed significantly $\left(t_{(10)}=3.617 ; p=0.012\right)$, and left frontal LV correlated positively with the response index on day $5(\mathrm{~N}=12$, Pearson's $r=0.703 ; \mathrm{p}=0.011)$ (Figure 23). Whole brain or regional LV did not correlate with baseline fatigue levels on any scale. 

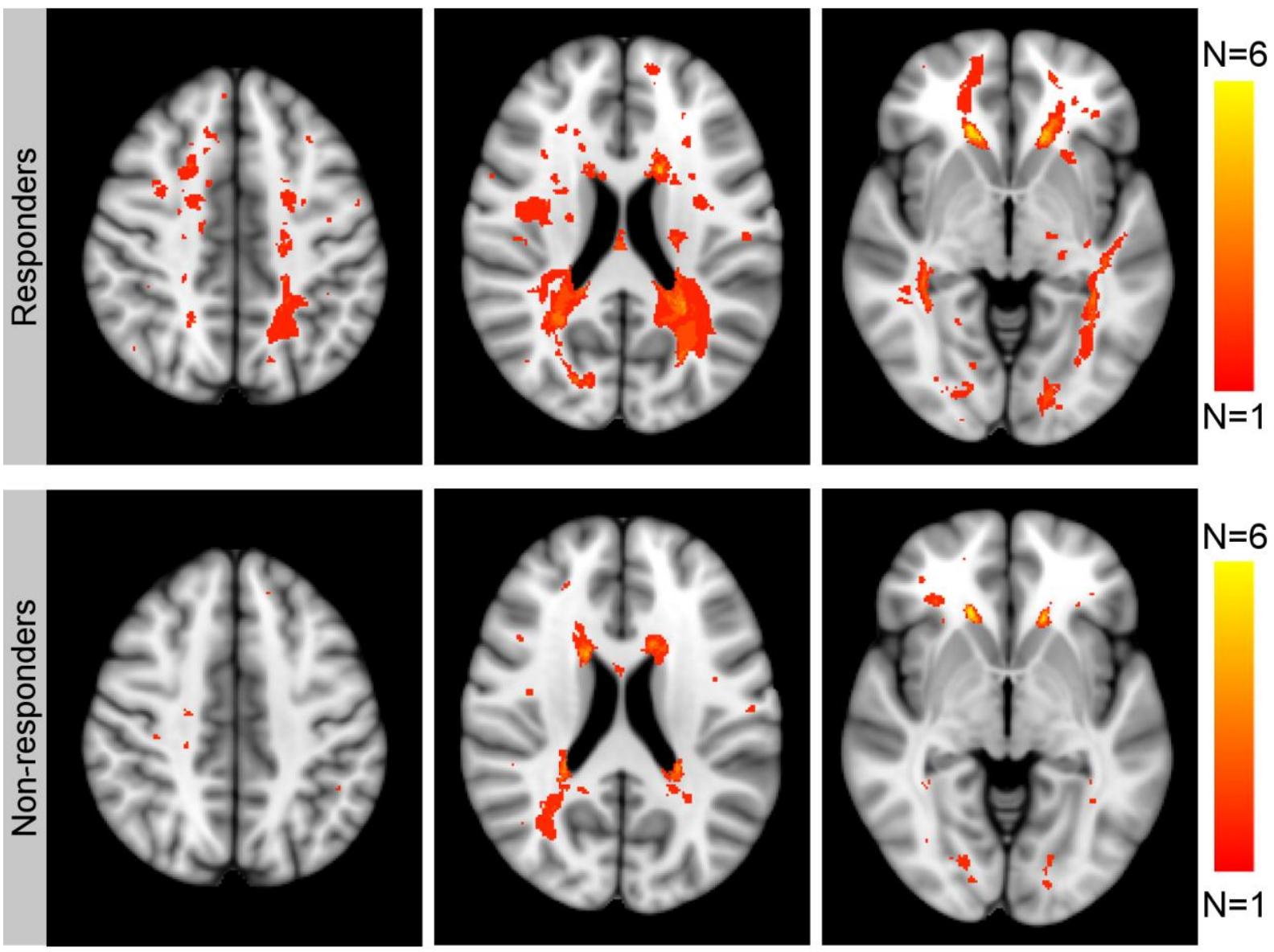

Figure 22. Lesion probability maps for responders $(\mathrm{N}=6)$ and non-responders $(\mathrm{N}=6)$. The lesion probability maps were obtained by summing the lesion masks in standard MNI space of patients within each group and dividing by the number of patients of the respective group. Images are shown in radiological convention.

A

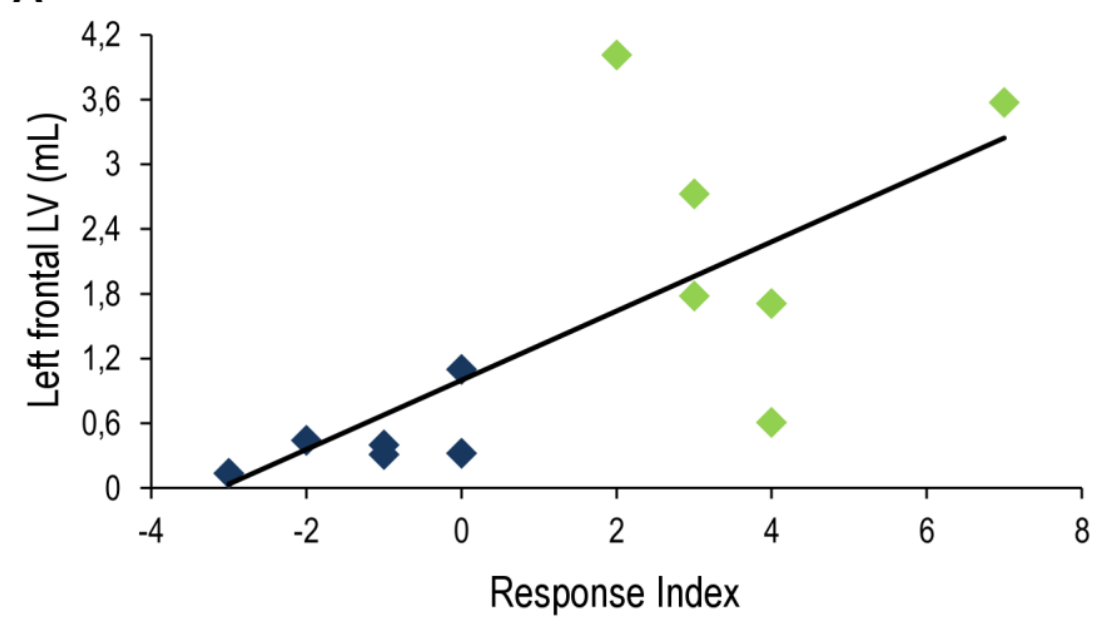

B

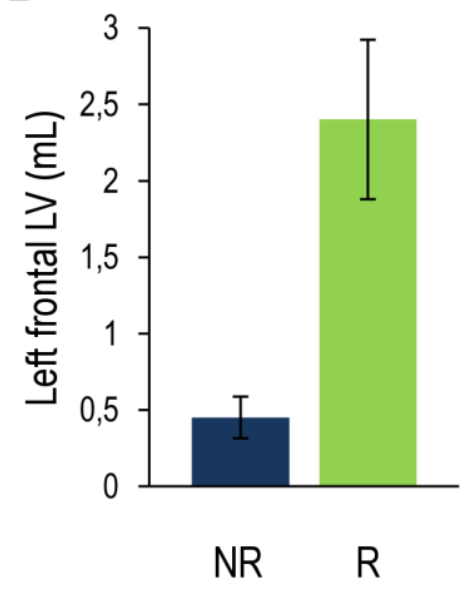

Figure 23. Lesion load in the left frontal area in responders and non-responders. A) Lesion load in the left frontal cortex correlated with the response index on day $5(p=0.011)$. B) Responders showed higher lesion load within the left frontal mask ( $p=0.012$ ). 


\subsection{Discussion}

The aim of this project was to improve fatigue symptoms in MS by anodal tDCS over the DLPFC. Two stimulation protocols were tested and neither induced an overall significant improvement in fatigue, in any scale. However, 7 (out of 13) patients from protocol 1 responded positively to the stimulation. Furthermore, in an exploratory analysis to investigate what differentiated responders from non-responders, it was observed that response to anodal tDCS correlated with left frontal lesion load. In the next paragraphs, the obtained results and possible limitations of the studies are discussed.

\subsubsection{Baseline fatigue}

Concerning the baseline fatigue scores, although the FSS values correlated moderately with the MFIS values, the correlation was not significant. Nevertheless, previous findings were replicated, in that the FSS scores correlated strongest with the values of the subscale MFISphy, but not with the MFISpsych and the MFIScog scores, which is an expected result as the FSS focuses predominantly on physical symptoms (Amtmann et al., 2012; Flachenecker et al., 2002). However, contrarily to previous reports, the MSFSS scores did not correlate with any other scale. Weaker correlations between the values of the MSFSS and the other fatigue scales have been described before (Flachenecker et al., 2002), probably due to the fact that the MSFSS targets very specific aspects of fatigue in MS, but does not efficiently quantify the severity of fatigue or its impact (Elbers et al., 2012). In protocol 2, the VAS values for fatigue did not correlate with the values of any other fatigue scale, perhaps because it encompasses all aspects of fatigue, instead of focusing more on a particular trait, although this is in contradiction with results available in the literature (Flachenecker et al., 2002) and more likely due to the small sample size. In addition, fatigue severity did not correlate with age, disease duration or EDSS scores. In previous studies it has been observed that fatigue severity is correlated to disability (Kroencke et al., 2000). However, due to the inclusion criteria, the patients selected for this study had overall low EDSS scores, which could explain why in this particular sample the EDSS and fatigue severity were not correlated.

The baseline fatigue levels did not correlate with lesion load, either global nor in one of the defined regions, regardless of the considered fatigue scale. Such result is in agreement with the observations of numerous studies in the literature, which were unable to find a significant association between fatigue and whole brain or regional lesion load from T2 or T1 structural 
images (Riccitelli et al., 2011a; Tartaglia et al., 2004; Tedeschi et al., 2007; van der Werf et al., 1998; Yaldizli et al., 2011), in spite of a few exceptions (Colombo et al., 2000; Sepulcre et al., 2009). More studies have reported increased GM atrophy in patients suffering from fatigue, particularly when separately analysing specific brain regions. For instance, GM atrophy of the left central sulcus and precentral gyrus was more pronounced in patients with fatigue according to the FSS scores (Riccitelli et al., 2011a), and a correlation of fatigue severity with frontal GM atrophy (Sepulcre et al., 2009) and atrophy of the corpus callosum (Yaldizli et al., 2011) have also been described. Thus, future studies with an appropriately sized sample should investigate preferably regional GM atrophy, include functional measures and relate these to potential effects of stimulation.

\subsubsection{Responsiveness to stimulation}

The overall results do not support a significant reduction of fatigue by prefrontal anodal tDCS, for either protocol. In the patients undergoing protocol 1, the scores of the MFIS decreased throughout the experiment regardless of stimulation, but even though the changes were stronger after anodal tDCS, they did not significantly differ from sham. The strongest response to protocol 2 was a modest decrease of the VAS for fatigue and of the MFIS, which however was not significantly different from the changes registered after sham stimulation.

It is somewhat surprising that anodal tDCS did not have stronger effects, given the wide range of successful therapeutic applications, particularly when stimulating over the prefrontal cortex (Flöel, 2013; M.-F. Kuo et al., 2013). However, several limitations could have prevented better results and will be discussed further on. Nevertheless, a subgroup of patients responded positively to the stimulation. Responders were defined using the change in perceived fatigue on day 5 , however it should be noted that in this group the changes were robust and long lasting, with perceived fatigue in the responder group showing reductions until day 30. Anodal tDCS could possibly have influenced fatigue in MS by inducing an increase in cortical excitability (Liebetanz et al., 2002; Nitsche and Paulus, 2000), as a weaker polarization of the neuronal membrane could partly counteract the deficient axonal conduction caused by demyelination that characterizes MS pathology. Considering that fMRI studies have shown that tDCS is able to increase resting-state functional connectivity between cortical regions and also with subcortical structures (Keeser et al., 2011; PeñaGómez et al., 2012; Polanía et al., 2012a, 2012b), another possibility is that the stimulation 
acted against the functional disruption, which has been associated with fatigue (DeLuca et al., 2009). Based on the results of tDCS experiments in rats (Rueger et al., 2012), it has also been suggest that tDCS can act by influencing inflammatory processes (Ferrucci et al., 2013), which may be involved in fatigue in MS (Braley and Chervin, 2010; Induruwa et al., 2012).

\subsubsection{Responders vs non-responders}

Given the heterogeneity of MS, and the several factors affecting fatigue itself, we explored if certain subject characteristics differed between responders and non-responders. We found that higher lesion load, specifically of the left frontal lobe, was associated with a positive response to stimulation. One possible explanation for the impact of lesion load on the response to tDCS, is the important role that anatomical features play with regard to the distribution of the electric field induced by NIBS techniques in the brain (Opitz et al., 2011; Salvador et al., 2010). The lesion load differences between responders and non-responders were found in left frontal region which lies between the electrodes, where the simulation of electric field distribution for our electrode montage predicts the higher electric fields and stimulation effects are expected to be the strongest. Theoretical predictions strongly suggest that anatomical features such as alterations in gray matter thickness and individual patterns of GM and WM lesions can affect the distribution of the induced electric field in the brain (Datta et al., 2010) and thus the efficacy of tDCS. Alternatively, one could speculate that patients with higher lesion load could have stronger compensation mechanisms in action (Penner et al., 2003), which could better benefit from the plasticity-inducing effects of anodal tDCS discussed above.

The only other factor that differed between responders and non-responders was baseline depression. We found that responders had higher baseline BDI scores. Given the positive results of anodal tDCS against depression abundantly described in the literature (Felipe Fregni et al., 2006b; Nitsche et al., 2009), it could be speculated that the changes in fatigue observed in responders are actually due to an anti-depressant effect of the stimulation. Indeed, fatigue has been found to correlate strongly with depression (Lerdal et al., 2007). However, in the present study the baseline BDI scores before anodal tDCS were low for both groups (responders: median $=6$, range: 4-13; non-responders: median $=4$, range: $2-5$ ), well below the diagnostic threshold for depression (cut-off BDI = 19). Furthermore, depression scores did not correlate with response index and HADS-D scores did not differ significantly 
between responders and non-responders. Also, the decrease in BDI scores was significant only after sham stimulation (whole group) and not significant in the responder group, which does not suggest an effect of tDCS on BDI scores, not even among responders. In addition, BDI baseline did not have an effect of changes in perceived fatigue on day 5 , according to the results of an ordinal logistic regression analysis. Thus, we consider that it is unlikely that the response to stimulation was determined by baseline depression levels, or caused by an antidepressant effect of anodal tDCS.

One other study, which has recently attempted to reduce fatigue symptoms among MS patients applying bilateral motor cortex tDCS also reports a positive response in a subset of patients (15 out of 23) (Ferrucci et al., 2013). The authors report a significant decrease in scores of the motor component of the Fatigue Impact Scale, but not in a VAS for fatigue. In this case, responders were younger than non-responders, which - as the authors suggested could facilitate plasticity induction by tDCS. Unfortunately, in this study the lesion load was not measured and so it cannot be used to compare with our findings.

\subsubsection{Fatigue scales results and limitations}

It is possible that the fatigue scales were not the most adequate tool for detecting alterations caused by tDCS. Although the FSS has been considered a reliable tool for detecting fatigue among MS patients (Flachenecker et al., 2002; Krupp et al., 1989), recent studies suggest it should be shortened as there is redundancy among items (Amtmann et al., 2012; Mills et al., 2009), and some items are biased towards factors such as age, disability and disease type (Mills et.al, 2009).

Furthermore, as confirmed by the correlation between the FSS and the MFISphy, the FSS scale mainly addresses physical functions, possibly the least affected by electrical stimulation over the DLPFC. A recent study, where patients with post-polio syndrome received anodal tDCS over the premotor cortex, also reported no significant reduction in FSS scores compared with sham (Acler et al., 2013). The MFIS has been shown to discriminate successfully between patients with and without fatigue (Flachenecker et al., 2002) and has low floor and ceiling effects (Amtmann et al., 2012) but the questionnaire considers a time window of 4 weeks and therefore its temporal resolution is inadequate for assessing changes in the intermediate days, e.g. in our study. Even though tendencies towards improvement could be seen in the FSS and MFIS, we observed a discrepancy between fatigue scales and 
change in perceived fatigue, even for responding patients, providing further evidence that the used fatigue scales were not ideally suited for our study, and might have under-rated changes. These factors might have contributed to the discrepancy between the observed changes in fatigue scales and the reported symptom reduction.

Because fatigue is multidimensional and results from contribution of different factors, it is possible that the standard scales do not satisfactorily picture the experienced symptoms and a compound scale such as the change in perceived fatigue better captures the overall fatigue individually experienced by the patients. However, this scale has been introduced by us and consequently it has not been formally tested for reliability or validity. In addition, it requires that patients compare their current fatigue level to that experienced several days before and therefore, asking directly for the fatigue level on each day, for instance with a 10-point VAS, might have been a better solution. However, since asking for the amount of actual fatigue will also at least implicitly include a relation to other time points (to rate severity you need some subjective fix points for no symptoms, and worst symptoms), and our main interest was the change of symptoms, and not absolute severity, we have chosen this format. Furthermore, the change in perceived fatigue has the advantage of making it relatively easy for the patients to identify the direction of change. Therefore, we implemented the VAS for fatigue on the second protocol. However, the VAS variation after stimulation did not correlate with the changes in perceived fatigue. This is surprising because both scales were expected to measure a change in overall fatigue, instead of the focus that some scales have on particular aspect of fatigue. Even though the sample is small, the negative result is probably due to the fact that stimulation had no robust effect. Thus, small variations in the scales do not reflect actual fatigue changes. Unfortunately, we do not have VAS results to compare with for the first protocol, which had a bigger sample size and more promising results.

\subsubsection{Stimulation parameters}

The absence of stronger treatment-effects can be due to the chosen stimulation parameters, such as stimulation intensity and duration, frequency and electrode size. Given the results presented by (Monte-Silva et al., 2013) when comparing several stimulation protocols, we hypothesized that stimulation applied twice daily would have had larger effects. Although the number of patients, which underwent the second stimulation protocol is very small, the results obtained so far do not support such hypothesis, and in our study protocol I seems to 
be more effective. It is also possible that stronger stimulation would have induced larger effects. For instance, Boggio and colleagues (2006b) have described stimulation intensitydependent effects of tDCS on working memory performance in patients with Parkinson's disease, where stronger stimulation was more effective. However, the situation might be complicated by non-linear effects of stimulation duration and intensity, as neither increased intensity nor increased duration necessarily lead to stronger or longer-lasting effects. Indeed, these studies it have reported that doubling the duration of anodal tDCS from 13 to 26 min caused reduced excitability and doubling the intensity of cathodal tDCS to $2 \mathrm{~mA}$ caused excitability increases (Batsikadze et al., 2013; Monte-Silva et al., 2013).

Nevertheless, it is unclear whether results collected in healthy populations or other neuropsychiatric diseases can be translated one-to-one to MS. Altered cortical excitability has been found in patients with MS (Thickbroom et al., 2005), which can influence the effect of tDCS (Antal et al., 2007) and systematic studies on optimal stimulation protocols in MS are not available. Anodal tDCS with $1 \mathrm{~mA}$ intensity over the motor cortex $\left(25 \mathrm{~cm}^{2}\right.$ anode, $50 \mathrm{~cm}^{2}$ cathode over contralateral forehead) was shown recently to modestly modulate corticospinal output and projection strength in MS patients (Cuypers et al., 2013b) but failed to consistently improve motor performance (Meesen et al., 2013). More encouraging results were found when stimulating with higher intensity: 6 out of 10 MS patients suffering from chronic neuropathic pain benefited from 5 consecutive days of anodal_tDCS over the motor cortex at $2 \mathrm{~mA}$ intensity (parallel group design, both electrodes $35 \mathrm{~cm}^{2}$, cathode over contralateral forehead), resulting in reduced pain levels by $50 \%$ or more after 4 weeks (Mori et al., 2010) and the same protocol improved tactile perception in MS patients with sensory deficits (Mori et al., 2013) Concerning motor fatigue, promising results have been reported describing that anodal tDCS over the motor cortex (10 min, $1.5 \mathrm{~mA}$, both electrodes $35 \mathrm{~cm}^{2}$, cathode over ipsilateral shoulder) decreased muscular fatigue in healthy subjects (Cogiamanian et al., 2007). Also, the positive effects recently described by Ferrucci et al. (2013) in 65\% of MS patients, were observed using bilateral anodal tDCS over the motor cortex (25 $\mathrm{cm}^{2}$, with a third electrode as extra-cephalic reference) at $1.5 \mathrm{~mA}$ stimulation intensity during 5 consecutive days, thus with a different montage and higher intensity than used in our study. The results described above suggest that increasing the intensity of the stimulation might have been more beneficial. 


\subsubsection{Placebo effect}

We observed a strong placebo effect that may have partially masked the real effect of tDCS. For instance, FSS scores for the whole patient group decreased only after sham stimulation mainly due to patients receiving sham stimulation first. Also, MFIS and depression scores during protocol 1 showed an interaction between order and the other factors, which can be attributed to a placebo effect. Placebo effects up to $40 \%$ are frequently observed in studies including patients with chronic pain or restless legs syndrome which, like fatigue, depend on subjective perception by the patients (Fulda and Wetter, 2008) With the applied crossover design, the response to the second block can be strongly influenced by the experiences during the first block, making it difficult to interpret the results. Therefore, future studies should replicate this finding with a between-subjects design, using an appropriately sized sample.

One other possible improvement to our study design would have been to systematically control for sleep disturbances, which can contribute to fatigue severity (Braley and Chervin, 2010). In a recent study, 3 weeks of bilateral anodal tDCS over the pre-motor cortex (1.5 mA, 15 min, cathode over the left shoulder) was able to slightly improve sleep and fatigue symptoms among patients with post-polio syndrome (Acler et al., 2013). Other possible shortcomings were avoided by our strict exclusion criteria, which minimize important confounders, such as minimum fatigue duration and relapses. Also, depression is often associated with fatigue (Kroencke et al., 2000) and would have been a serious confounder, since tDCS can reduce depression (Nitsche et al., 2009). Besides excluding patients with depression, we confirmed that this symptom was not affected by the stimulation throughout the intervention. Furthermore, patients were not taking medication known to influence fatigue, and all had the same disease course, which plays a role in cortical excitability and fatigue severity (Leocani et al., 2001). In addition, we have confirmed that the used sham method is valid even for longer stimulation period and repeated sessions, at least for naive subjects (Ambrus et al., 2012). Importantly, our results concerning NSE levels also support that of 5 days consecutive stimulation at $1 \mathrm{~mA}$ induces no neuronal damage.

In summary, we did not find a robust effect of anodal tDCS on MS fatigue, but the positive response of a subset of patients is encouraging. Thus, this exploratory study supports the potential of anodal tDCS on MS-fatigue therapy, but more research is needed to understand which factors to consider in tailoring the intervention to each patient's needs, and to understand its mechanisms of action in larger detail. 


\section{Conclusions}

\subsection{Summary of results}

In the presented studies, diverse methods of tES were tested for their ability to modulate behaviour in healthy subjects and in MS patients.

In the first project, we studied the effects of tES on healthy subjects performing a visuomotor coordination task. The participants received anodal tDCS, cathodal tDCS, hf-tRNS, lf-tRNS or sham stimulation with the electrodes placed over the M1 and the contralateral forehead for 10 minutes, while learning a complex motor task guided by visual feedback. Contrary to the expectations, we observed that none of the stimulation methods induced significant changes in task performance, either during the stimulation period or up to 20 minutes after it. Also, the stimulation did not significantly modulate motor-related or performance-related brain activation that measured with fMRI during the experiment, except for a moderate decrease in activation of areas of the frontal cortex and precuneous caused by hf-tRNS. In addition, there was a tendency for hf-tRNS to improve performance and for lf-tRNS to worsen it. However, as discussed, one limitation of this study was the different baseline performance levels between groups, which hinders a more complete discussion of the stimulation effects.

In the second project, we tested the therapeutic application of anodal tDCS against fatigue in patients with MS. We applied anodal tDCS over the left DLPFC with two different stimulation protocols (daily 20 min for 5 consecutive days or 20 min twice on only 1 day) and tracked fatigue changes over the course of approximately one month using several fatigue scales. Overall, the stimulation did not have a significant impact on fatigue levels measured with any scale. Although the sample sizes, particularly of the second protocol, are small, the results suggest that five consecutive days of stimulation is a better suited approach. Nevertheless, a subset of patients responded positively to the first stimulation protocol, with long lasting reduction of fatigue. Compared to non-responders, these patients had higher left frontal lesion load which could possibly modulate the stimulation effects. Thus, anodal tDCS holds promise as therapeutic intervention against fatigue in MS. The main limitation of the study was the strong placebo effect, which could be corrected by using parallel groups and the small sample size, which prevents us from withdrawing from the results observations as conclusive as desirable. 


\subsection{Final conclusions and future directions}

As evident in the literature, the data from the first study suggest that results obtained in physiology studies concerning the stimulation effects on cortical excitability are not easily translated to behavioural measurements, or even from one behavioural task to another. More than the characteristics of the stimulation, the effects of tDCS seem to strongly depend on factors such as the combination of the stimulation protocol with a behavioural paradigm, the state of the brain or other individual characteristics. In spite of the undeniable potential of tES techniques, there is still great room for improvement, and systematic studies to devise optimized stimulation protocols for specific applications are needed. Considering that the effect of the stimulation is often modest and subject to great variability, it is fundamental that any future study will implement a careful double-blind design and a big enough sample size to avoid being underpowered.

Results from the second study further underline the impact of individual anatomical brain characteristics on the effects induced by tDCS. TDCS studies on patients with brain damage should greatly benefit from including a structural MRI in their design for creating individualized head models for theoretical predictions of the electric field distribution in the brain during stimulation. Although it is not yet clear how to use these predictions for future study design, they can contribute to a more complete interpretation of the results. In addition to anatomical differences, which can modify the induced electric field, patients with neuropsychiatric disorders often show altered brain excitability and plasticity mechanisms. These can results in an altered reaction to the stimulation and call for adjustments on parameters such as current intensity. Future studies should focus on adapting methods obtained in healthy populations to specific patients groups or even a personalized application of the stimulation.

Ultimately, a better knowledge of the mechanisms by which tES acts on the brain is of paramount importance to explain the interaction of the parameters influencing the stimulation effects. The understanding of such factors can contribute to an informed and efficient application of tES techniques to utilize their full potential in basic and clinical research. 


\section{Appendix 1 - Fatigue scales}

FSS

1) My motivation is lower when I am fatigued.

2) Exercise brings on my fatigue.

3) I am easily fatigued.

4) Fatigue interferes with my physical functioning.

5) Fatigue causes frequent problems for me.

6) My fatigue prevents sustained physical functioning.

7) Fatigue interferes with carrying out certain duties and responsibilities.

8) Fatigue is among my three most disabling symptoms.

9) Fatigue interferes with my work, family, or social life.

\section{MSFSS}

1) Heat brings on fatigue.

2) Long periods of inactivity bring on fatigue.

3) Stress brings on fatigue.

4) Depression brings on fatigue.

5) Cool temperatures lessen fatigue.

6) Positive experiences lessen fatigue.

\section{MFIS}

1) I feel less alert.

2) I have difficulty paying attention for a long period of time.

3) I feel like I cannot think clearly.

4) I am more clumsy and uncoordinated.

5) I find that I am more forgetful.

6) I have to limit my physical activities.

7) I am less motivated to do anything that requires physical effort.

8) I am less motivated to engage in social activities.

9) I am limited to do anything outside my home.

10) I have trouble maintaining physical effort for long periods.

11) I find it difficult to make decisions.

12) I am less motivated to do anything that requires thinking.

13) I feel weak.

14) My physical discomfort is increased.

15) I am less able to finish tasks that require thinking.

16) I find it difficult to organize my thoughts when I am doing things at home or at work.

17) I am less able to complete tasks that require physical effort.

18) I feel slowed down in my thinking.

19) I find it hard to concentrate.

20) I have to limit my physical activities.

21) I require more frequent or longer periods of rest. 


\section{Bibliography}

Acler, M., Bocci, T., Valenti, D., Turri, M., Priori, A., Bertolasi, L., 2013. Transcranial Direct Current Stimulation (tDCS) for sleep disturbances and fatigue in patients with post-polio syndrome. Restor. Neurol. Neurosci. 31, 661-668.

Amadi, U., Ilie, A.S., Johansen-Berg, H., Stagg, C.J., 2013. Polarity-specific effects of motor transcranial direct current stimulation on fMRI resting state networks. Neuroimage (in press).

Amassian, V.E., Cracco, R.Q., Maccabee, P.J., Cracco, J.B., Rudell, A.P., Eberle, L., 1998. Transcranial Magnetic Stimulation in Study of the Visual Pathway. J. Clin. Neurophysiol. 15.

Ambrus, G.G., Al-Moyed, H., Chaieb, L., Sarp, L., Antal, A., Paulus, W., 2012. The fade-in - Short stimulation - Fade out approach to sham tDCS - Reliable at $1 \mathrm{~mA}$ for naïve and experienced subjects, but not investigators. Brain Stimul. 5, 499-504.

Ambrus, G.G., Paulus, W., Antal, A., 2010. Cutaneous perception thresholds of electrical stimulation methods: comparison of tDCS and tRNS. Clin. Neurophysiol. 121, 1908-1914.

Ambrus, G.G., Zimmer, M., Kincses, Z.T., Harza, I., Kovács, G., Paulus, W., Antal, A., 2011. The enhancement of cortical excitability over the DLPFC before and during training impairs categorization in the prototype distortion task. Neuropsychologia 49, 1974-1980.

Amtmann, D., Bamer, A.M., Noonan, V., Lang, N., Cook, K.F., 2012. Comparison of the psychometric properties of two fatigue scales in multiple sclerosis. Rehabil Psychol. 57, 159-166.

Anbeek, P., Vincken, K.L., van Osch, M.J.P., Bisschops, R.H.C., van der Grond, J., 2004. Probabilistic segmentation of white matter lesions in MR imaging. Neuroimage 21, 1037-1044.

Andersson, J.L.R., Jenkinson, M., Smith, S., 2007. Non-linear registration aka Spatial normalisation FMRIB Technial Report TRo7JA2.

Antal, A., Begemeier, S., Nitsche, M.A., Paulus, W., 2008a. Prior state of cortical activity influences subsequent practicing of a visuomotor coordination task. Neuropsychologia 46, 3157-3161.

Antal, A., Bikson, M., Datta, A., Lafon, B., Dechent, P., Parra, L.C., Paulus, W., 2014. Imaging artifacts induced by electrical stimulation during conventional fMRI of the brain. Neuroimage 85, 10401047 .

Antal, A., Boros, K., Poreisz, C., Chaieb, L., Terney, D., Paulus, W., 2008b. Comparatively weak aftereffects of transcranial alternating current stimulation (tACS) on cortical excitability in humans. Brain Stimul. 1, 97-105.

Antal, A., Nitsche, M.A., Kincses, T.Z., Kruse, W., Hoffmann, K.-P., Paulus, W., 2004a. Facilitation of visuo-motor learning by transcranial direct current stimulation of the motor and extrastriate visual areas in humans. Eur. J. Neurosci. 19, 2888-2892.

Antal, A., Nitsche, M.A., Kruse, W., Kincses, T.Z., Hoffmann, K.-P., Paulus, W., 2004b. Direct current stimulation over $\mathrm{V}_{5}$ enhances visuomotor coordination by improving motion perception in humans. J. Cogn. Neurosci. 16, 521-527.

Antal, A., Polania, R., Schmidt-Samoa, C., Dechent, P., Paulus, W., 2011. Transcranial direct current stimulation over the primary motor cortex during fMRI. Neuroimage 55, 590-596.

Antal, A., Terney, D., Kühnl, S., Paulus, W., 2010. Anodal Transcranial Direct Current Stimulation of the Motor Cortex Ameliorates Chronic Pain and Reduces Short Intracortical Inhibition. J. Pain Symptom Manage. 39, 890-903. 
Antal, A., Terney, D., Poreisz, C., Paulus, W., 2007. Towards unravelling task-related modulations of neuroplastic changes induced in the human motor cortex. Eur. J. Neurosci. 26, 2687-2691.

Ardolino, G., Bossi, B., Barbieri, S., Priori, A., 2005. Non-synaptic mechanisms underlie the aftereffects of cathodal transcutaneous direct current stimulation of the human brain. J. Physiol. 568, $653-663$.

Aydin, K., Ucar, A., Oguz, K.K., Okur, O.O., Agayev, A., Unal, Z., Yilmaz, S., Ozturk, C., 2007. Increased gray matter density in the parietal cortex of mathematicians: a voxel-based morphometry study. AJNR. Am. J. Neuroradiol. 28, 1859-1864.

Bakshi, R., Miletich, R., Henschel, K., Shaikh, Z., Janardhan, V., Wasay, M., Stengel, L., Ekes, R., Kinkel, P., 1999. Fatigue in multiple sclerosis: cross-sectional correlation with brain MRI findings in 71 patients. Neurology 53, 1151-1153.

Barkhof, F., 2002. The clinico-radiological paradox in multiple sclerosis revisited. Curr. Opin. Neurol. $15,239-245$.

Batsikadze, G., Moliadze, V., Paulus, W., Kuo, M.-F., Nitsche, M.A., 2013. Partially non-linear stimulation intensity-dependent effects of direct current stimulation on motor cortex excitability in humans. J. Physiol. 7, 1987-2000.

Battaglini, M., Jenkinson, M., De Stefano, N., 2012. Evaluating and reducing the impact of white matter lesions on brain volume measurements. Hum. Brain Mapp. 33, 2062-2071.

Baudewig, J., Nitsche, M.A., Paulus, W., Frahm, J., 2001. Regional modulation of BOLD MRI responses to human sensorimotor activation by transcranial direct current stimulation. Magn. Reson. Med. 45, 196-201.

Beckmann, C.F., DeLuca, M., Devlin, J.T., Smith, S.M., 2005. Investigations into resting-state connectivity using independent component analysis. Philos. Trans. R. Soc. B Biol. Sci. 360, 10011013 .

Beckmann, M., Johansen-Berg, H., Rushworth, M.F.S., 2009. Connectivity-based parcellation of human cingulate cortex and its relation to functional specialization. J. Neurosci. 29, 1175-1190.

Bermudez, P., Zatorre, R.J., 2005. Differences in gray matter between musicians and nonmusicians. Ann. N. Y. Acad. Sci. 1060, 395-399.

Bester, M., Lazar, M., Petracca, M., Babb, J.S., Herbert, J., Grossman, R.I., Inglese, M., 2013. Tractspecific white matter correlates of fatigue and cognitive impairment in benign multiple sclerosis. J. Neurol. Sci. 330, 61-66.

Bikson, M., Inoue, M., Akiyama, H., Deans, J.K., Fox, J.E., Miyakawa, H., Jefferys, J.G.R., 2004. Effects of uniform extracellular DC electric fields on excitability in rat hippocampal slices in vitro. J. Physiol. 557, 175-190.

Biswal, B., Yetkin, F.Z., Haughton, V.M., Hyde, J.S., 1995. Functional Connectivity in the Motor Cortex of Resting Human Brain Using Echo-Planar MRI. Magn. Reson. Med. 34, 537-541.

Bliss, T., Cooke, S., 2011. Long-term potentiation and long-term depression: a clinical perspective. Clinics 66, 3-17.

Boggio, P.S., Castro, L.O., Savagim, E.A., Braite, R., Cruz, V.C., Rocha, R.R., Rigonatti, S.P., Silva, M.T.A., Fregni, F., 2006a. Enhancement of non-dominant hand motor function by anodal transcranial direct current stimulation. Neurosci. Lett. 404, 232-236.

Boggio, P.S., Ferrucci, R., Mameli, F., Martins, D., Martins, O., Vergari, M., Tadini, L., Scarpini, E., Fregni, F., Priori, A., 2012. Prolonged visual memory enhancement after direct current stimulation in Alzheimer's disease. Brain Stimul. 5, 223-230. 
Boggio, P.S., Ferrucci, R., Rigonatti, S.P., Covre, P., Nitsche, M., Pascual-leone, A., Fregni, F., 2006b. Effects of transcranial direct current stimulation on working memory in patients with Parkinson ' s disease. J. Neurol. Sci. 249, $31-38$.

Boggio, P.S., Khoury, L.P., Martins, D.C.S., Martins, O.E.M.S., de Macedo, E.C., Fregni, F., 2009. Temporal cortex direct current stimulation enhances performance on a visual recognition memory task in Alzheimer disease. J. Neurol. Neurosurg. Psychiatry 80 , 444-447.

Boggio, P.S., Rigonatti, S.P., Ribeiro, R.B., Myczkowski, M.L., Nitsche, M.A., Pascual-Leone, A., Fregni, F., 2008a. A randomized, double-blind clinical trial on the efficacy of cortical direct current stimulation for the treatment of major depression. Int. J. Neuropsychopharmacol. 11, 249-254.

Boggio, P.S., Sultani, N., Fecteau, S., Merabet, L., Mecca, T., Pascual-Leone, A., Basaglia, A., Fregni, F., 2008b. Prefrontal cortex modulation using transcranial DC stimulation reduces alcohol craving: A double-blind, sham-controlled study. Drug Alcohol Depend. 92, 55-60.

Braley, T.J., Chervin, R.D., 2010. Fatigue in multiple sclerosis: mechanisms, evaluation, and treatment. Sleep 33, 1061-1067.

Brown, T.R., Kraft, G.H., 2005. Exercise and Rehabilitation for Individuals with Multiple Sclerosis. Phys. Med. Rehabil. Clin. N. Am. 16, 513-555.

Brunelin, J., Mondino, M., Gassab, L., Haesebaert, F., Gaha, L., Suaud-Chagny, M.F., Saoud, M., Mechri, A., Poulet, E., 2012. Examining Transcranial Direct-Current Stimulation (tDCS) as a Treatment for Hallucinations in Schizophrenia. Am J Psychiatry 169, 719-724.

Brunoni, A.R., Ferrucci, R., Bortolomasi, M., Vergari, M., Tadini, L., Boggio, P.S., Giacopuzzi, M., Barbieri, S., Priori, A., 2011. Transcranial direct current stimulation (tDCS) in unipolar vs. bipolar depressive disorder. Prog. Neuro-Psychopharmacology Biol. Psychiatry 35, 96-101.

Brunoni, A.R., Valiengo, L., Baccaro, A., Al, E., 2013. The sertraline vs electrical current therapy for treating depression clinical study: Results from a factorial, randomized, controlled trial. JAMA Psychiatry 70, 383-391.

Bullmore, E., Sporns, O., 2009. Complex brain networks: graph theoretical analysis of structural and functional systems. Nat. Rev. Neurosci. 10, 186-198.

Calhoun, V.D., Adali, T., Pearlson, G.D., Pekar, J.J., 2001. A method for making group inferences from functional MRI data using independent component analysis. Hum. Brain Mapp. 14, 140-151.

Cappelletti, M., Gessaroli, E., Hithersay, R., Mitolo, M., Didino, D., Kanai, R., Cohen Kadosh, R., Walsh, V., 2013. Transfer of Cognitive Training across Magnitude Dimensions Achieved with Concurrent Brain Stimulation of the Parietal Lobe. J. Neurosci. 33, 14899-14907.

Cavanna, A.E., Trimble, M.R., 2006. The precuneus: a review of its functional anatomy and behavioural correlates. Brain 129, 564-583.

Chaieb, L., Kovacs, G., Cziraki, C., Greenlee, M., Paulus, W., Antal, A., 2009. Short-duration transcranial random noise stimulation induces blood oxygenation level dependent response attenuation in the human motor cortex. Exp. brain Res. 198, 439-444.

Chalder, T., Berelowitz, G., Pawlikowska, T., Watts, L., Wessely, S., Wright, D., Wallace, E., 1993. Development of a fatigue scale. J Psychosom Res 37, 147-153.

Chaudhuri, A., Behan, P.O., 2004. Fatigue in neurological disorders. Lancet 363, 978-988.

Citri, A., Malenka, R.C., 2008. Synaptic plasticity: multiple forms, functions, and mechanisms. Neuropsychopharmacology 33, 18-41. 
Cogiamanian, F., Marceglia, S., Ardolino, G., Barbieri, S., Priori, A., 2007. Improved isometric force endurance after transcranial direct current stimulation over the human motor cortical areas. Eur. J. Neurosci. 26, 242-249.

Collingridge, G.L., Peineau, S., Howland, J.G., Wang, Y.T., 2010. Long-term depression in the CNS. Nat. Rev. Neurosci. 11, 459-473.

Colombo, B., Martinelli Boneschi, F., Rossi, P., Rovaris, M., Maderna, L., Filippi, M., Comi, G., 2000. MRI and motor evoked potential findings in nondisabled multiple sclerosis patients with and without symptoms of fatigue. J. Neurol. 247, 506-509.

Comi, G., Leocani, L., Rossi, P., Colombo, B., 2001. Physiopathology and treatment of fatigue in multiple sclerosis. J. Neurol. 248, 174-179.

Cooke, S.F., Bliss, T.V.P., 2006. Plasticity in the human central nervous system. Brain 129, 1659-1673.

Cramer, S.C., Sur, M., Dobkin, B.H., O’Brien, C., Sanger, T.D., Trojanowski, J.Q., Rumsey, J.M., Hicks, R., Cameron, J., Chen, D., Chen, W.G., Cohen, L.G., deCharms, C., Duffy, C.J., Eden, G.F., Fetz, E.E., Filart, R., Freund, M., Grant, S.J., Haber, S., Kalivas, P.W., Kolb, B., Kramer, A.F., Lynch, M., Mayberg, H.S., McQuillen, P.S., Nitkin, R., Pascual-Leone, A., Reuter-Lorenz, P., Schiff, N., Sharma, A., Shekim, L., Stryker, M., Sullivan, E. V, Vinogradov, S., 2011. Harnessing neuroplasticity for clinical applications. Brain 134, 1591-609.

Cuypers, K., Leenus, D.J.F., van den Berg, F.E., Nitsche, M.A., Thijs, H., Wenderoth, N., Meesen, R.L.J., 2013a. Is motor learning mediated by tDCS intensity? PLoS One 8, e67344.

Cuypers, K., Leenus, D.J.F., Van Wijmeersch, B., Thijs, H., Levin, O., Swinnen, S.P., Meesen, R.L.J., 2013b. Anodal tDCS increases corticospinal output and projection strength in multiple sclerosis. Neurosci. Lett. 554, 151-155.

Datta, A., Bikson, M., Fregni, F., 2010. Transcranial direct current stimulation in patients with skull defects and skull plates: high-resolution computational FEM study of factors altering cortical current flow. Neuroimage 52, 1268-1278.

Dayan, E., Cohen, L.G., 2011. Neuroplasticity subserving motor skill learning. Neuron 72, 443-454.

De Luca, M., Beckmann, C.F., De Stefano, N., Matthews, P.M., Smith, S.M., 2006. fMRI resting state networks define distinct modes of long-distance interactions in the human brain. Neuroimage 29, 1359-1367.

De Vries, M.H., Barth, A.C.R., Maiworm, S., Knecht, S., Zwitserlood, P., Flöel, A., 2009. Electrical Stimulation of Broca's Area Enhances Implicit Learning of an Artificial Grammar. J. Cogn. Neurosci. 22, 2427-2436.

DeLuca, J., Genova, H.M., Capili, E.J., Wylie, G.R., 2009. Functional Neuroimaging of Fatigue. Phys. Med. Rehabil. Clin. N. Am. 20, 325-337.

DeLuca, J., Genova, H.M., Hillary, F.G., Wylie, G., 2008. Neural correlates of cognitive fatigue in multiple sclerosis using functional MRI. J. Neurol. Sci. 270, 28-39.

Doyon, J., Benali, H., 2005. Reorganization and plasticity in the adult brain during learning of motor skills. Curr. Opin. Neurobiol. 15, 161-167.

Draganski, B., Gaser, C., Kempermann, G., Kuhn, H.G., Winkler, J., Büchel, C., May, A., 2006. Temporal and spatial dynamics of brain structure changes during extensive learning. J. Neurosci. $26,6314-6317$.

Ebert, D.H., Greenberg, M.E., 2013. Activity-dependent neuronal signalling and autism spectrum disorder. Nature 493, 327-37. 
Edwards, D., Cortes, M., Datta, A., Minhas, P., Wassermann, E.M., Bikson, M., 2013. Physiological and modeling evidence for focal transcranial electrical brain stimulation in humans: A basis for highdefinition tDCS. Neuroimage 74, 266-275.

Elbers, R.G., Rietberg, M.B., van Wegen, E.E.H., Verhoef, J., Kramer, S.F., Terwee, C.B., Kwakkel, G., 2012. Self-report fatigue questionnaires in multiple sclerosis, Parkinson's disease and stroke: a systematic review of measurement properties. Qual. Life Res. 21, 925-944.

Engström, M., Flensner, G., Landtblom, A.-M., Ek, A.-C., Karlsson, T., 2013. Thalamo-striato-cortical determinants to fatigue in multiple sclerosis. Brain Behav. 3, 715-728.

Faber, M., Vanneste, S., Fregni, F., De Ridder, D., 2012. Top down prefrontal affective modulation of tinnitus with multiple sessions of tDCS of dorsolateral prefrontal cortex. Brain Stimul. 5, 492498.

Fauvel, B., Groussard, M., Chételat, G., Fouquet, M., Landeau, B., Eustache, F., Desgranges, B., Platel, H., 2014. Morphological brain plasticity induced by musical expertise is accompanied by modulation of functional connectivity at rest. Neuroimage (in press).

Ferrucci, R., Mameli, F., Guidi, I., Mrakic-Sposta, S., Vergari, M., Marceglia, S., Cogiamanian, F., Barbieri, S., Scarpini, E., Priori, A., 2008. Transcranial direct current stimulation improves recognition memory in Alzheimer disease. Neurol. 71, 493-498.

Ferrucci, R., Vergari, M., Cogiamanian, F., Bocci, T., Ciocca, M., Tomasini, E., De Riz, M., Scarpini, E., Priori, A., 2013. Transcranial direct current stimulation (tDCS) for fatigue in multiple sclerosis. NeuroRehabilitation (in press).

Fertonani, A., Pirulli, C., Miniussi, C., 2011. Random noise stimulation improves neuroplasticity in perceptual learning. J. Neurosci. 31, 15416-15423.

Filippi, M., Evangelou, N., Kangarlu, A., Inglese, M., Mainero, C., Horsfield, M. a, Rocca, M. a, 2014. Ultra-high-field MR imaging in multiple sclerosis. J. Neurol. Neurosurg. Psychiatry 85, 60-66.

Filippi, M., Rocca, M., 2011. MR imaging of multiple sclerosis. Radiology 259, 659-681.

Filippi, M., Rocca, M.A., Benedict, R.H.B., DeLuca, J., Geurts, J.J.G., Rombouts, S.A.R.B., Ron, M., Comi, G., 2010. The contribution of MRI in assessing cognitive impairment in multiple sclerosis. Neurology 75, 2121-2128.

Filippi, M., Rocca, M.A., Colombo, B., Falini, A., Codella, M., Scotti, G., Comi, G., 2002. Functional magnetic resonance imaging correlates of fatigue in multiple sclerosis. Neuroimage $15,559-567$.

Flachenecker, P., Kümpfel, T., Kallmann, B., Gottschalk, M., Grauer, O., Rieckmann, P., Trenkwalder, C., Toyka, K. V, 2002. Fatigue in multiple sclerosis: a comparison of different rating scales and correlation to clinical parameters. Mult. Scler. 8, 523-526.

Flöel, A., 2013. tDCS-enhanced motor and cognitive function in neurological diseases. Neuroimage 85, Part 3, 934-947.

Floyer-Lea, A., Matthews, P.M., 2004. Changing brain networks for visuomotor control with increased movement automaticity. J. Neurophysiol. 92, 2405-2412.

Floyer-Lea, A., Matthews, P.M., 2005. Distinguishable brain activation networks for short- and longterm motor skill learning. J. Neurophysiol. 94, 512-518.

Fregni, Felipe, Boggio, P.S., Lima, M.C., Ferreira, M.J.L., Wagner, T., Rigonatti, S.P., Castro, A.W., Souza, D.R., Riberto, M., Freedman, S.D., Nitsche, M.A., Pascual-Leone, A., 2006a. A shamcontrolled, phase II trial of transcranial direct current stimulation for the treatment of central pain in traumatic spinal cord injury. Pain 122, 197-209. 
Fregni, Felipe, Boggio, P.S., Nitsche, M.A., Marcolin, M.A., Rigonatti, S.P., Pascual-Leone, A., $2006 \mathrm{~b}$. Treatment of major depression with transcranial direct current stimulation. Bipolar Disord. 8, 203-204.

Fregni, Felipe, Gimenes, R., Valle, A.C., Ferreira, M.J.L., Rocha, R.R., Natalle, L., Bravo, R., Rigonatti, S.P., Freedman, S.D., Nitsche, M.A., Pascual-Leone, A., Boggio, P.S., 2006c. A randomized, sham-controlled, proof of principle study of transcranial direct current stimulation for the treatment of pain in fibromyalgia. Arthritis Rheum. 54, 3988-3998.

Fregni, F., Liguori, P., Fecteau, S., 2008. Cortical stimulation of the prefrontal cortex with transcranial direct current stimulation reduces cue-provoked smoking craving: a randomized, shamcontrolled study. J. Clin. Psychiatry 69, 32-40.

Fregni, F, Marcondes, R., Boggio, P.S., Marcolin, M.A., Rigonatti, S.P., Sanchez, T.G., Nitsche, M.A., Pascual-Leone, A., 2006. Transient tinnitus suppression induced by repetitive transcranial magnetic stimulation and transcranial direct current stimulation. Eur. J. Neurol. 13, 996-1001.

Fulda, S., Wetter, T.C., 2008. Where dopamine meets opioids: a meta-analysis of the placebo effect in restless legs syndrome treatment studies. Brain 131, 902-917.

Galea, J.M., Vazquez, A., Pasricha, N., de Xivry, J.-J.O., Celnik, P., 2011. Dissociating the roles of the cerebellum and motor cortex during adaptive learning: the motor cortex retains what the cerebellum learns. Cereb. Cortex 21, 1761-1770.

Gandiga, P.C., Hummel, F.C., Cohen, L.G., 2006. Transcranial DC stimulation (tDCS): A tool for double-blind sham-controlled clinical studies in brain stimulation. Clin. Neurophysiol. 117, 845850 .

Ganguly, K., Poo, M.-M., 2013. Activity-dependent neural plasticity from bench to bedside. Neuron 80, 729-741.

Genova, H.M., Rajagopalan, V., DeLuca, J., Das, A., Binder, A., Arjunan, A., Chiaravalloti, N., Wylie, G., 2013. Examination of Cognitive Fatigue in Multiple Sclerosis using Functional Magnetic Resonance Imaging and Diffusion Tensor Imaging. PLoS One 8, e78811.

Giovannoni, G., 2006. Multiple sclerosis related fatigue. J. Neurol. Neurosurg. Psychiatry 77, 2-3.

Heesen, C., Nawrath, L., Reich, C., Bauer, N., Schulz, K.-H., Gold, S.M., 2006. Fatigue in multiple sclerosis: an example of cytokine mediated sickness behaviour? J. Neurol. Neurosurg. Psychiatry 77, 34-39.

Herholz, S.C., Zatorre, R.J., 2012. Musical training as a framework for brain plasticity: behavior, function, and structure. Neuron 76, 486-502.

Holland, R., Leff, A.P., Josephs, O., Galea, J.M., Desikan, M., Price, C.J., Rothwell, J.C., Crinion, J., 2011. Speech facilitation by left inferior frontal cortex stimulation. Curr. Biol. 21, 1403-1407.

Hulst, H.E., Schoonheim, M.M., Roosendaal, S.D., Popescu, V., Schweren, L.J.S., van der Werf, Y.D., Visser, L.H., Polman, C.H., Barkhof, F., Geurts, J.J.G., Werf, Y.D. Van Der, 2012. Functional adaptive changes within the hippocampal memory system of patients with multiple sclerosis. Hum. Brain Mapp. 33, 2268-2280.

Hyde, K.L., Lerch, J., Norton, A., Forgeard, M., Winner, E., Evans, A.C., Schlaug, G., 2009. Musical training shapes structural brain development. J. Neurosci. 29, 3019-3025.

Induruwa, I., Constantinescu, C.S., Gran, B., 2012. Fatigue in multiple sclerosis - a brief review. J. Neurol. Sci. 323, 9-15.

Inglese, M., Park, S., Johnson, G., Babb, J.S., Miles, L., Jaggi, H., Herbert, J., Grossman, R.I., 2007. Deep Gray Matter Perfusion in Multiple Sclerosis. Arch. Neurol. 64, 196-202. 
Jacobson, L., Goren, N., Lavidor, M., Levy, D.A., 2012a. Oppositional transcranial direct current stimulation (tDCS) of parietal substrates of attention during encoding modulates episodic memory. Brain Res. 1439, 66-72.

Jacobson, L., Koslowsky, M., Lavidor, M., 2012b. tDCS polarity effects in motor and cognitive domains: a meta-analytical review. Exp. Brain Res. 216, 1-10.

Janardhan, V., Bakshi, R., 2002. Quality of life in patients with multiple sclerosis: The impact of fatigue and depression. J. Neurol. Sci. 205, 51-58.

Jenkinson, M., Bannister, P., Brady, M., Smith, S., 2002. Improved Optimization for the Robust and Accurate Linear Registration and Motion Correction of Brain Images. Neuroimage 17, 825-841.

Jenkinson, M., Beckmann, C.F., Behrens, T.E.J., Woolrich, M.W., Smith, S.M., 2012. Fsl. Neuroimage $62,782-790$.

Jenkinson, M., Smith, S., 2001. A global optimisation method for robust affine registration of brain images. Med. Image Anal. 5, 143-156.

Kadosh, R.C., Soskic, S., Iuculano, T., Kanai, R., Walsh, V., 2010. Modulating neuronal activity produces specific and long-lasting changes in numerical competence. Curr. Biol. 20, 2016-2020.

Keeser, D., Meindl, T., Bor, J., Palm, U., Pogarell, O., Mulert, C., Brunelin, J., Möller, H.-J., Reiser, M., Padberg, F., 2011. Prefrontal transcranial direct current stimulation changes connectivity of resting-state networks during fMRI. J. Neurosci. 31, 15284-15293.

Kim, C.R., Kim, D.-Y., Kim, L.S., Chun, M.H., Kim, S.J., Park, C.H., 2012. Modulation of cortical activity after anodal transcranial direct current stimulation of the lower limb motor cortex: a functional MRI study. Brain Stimul. 5, 462-467.

Krakauer, J.W., Mazzoni, P., 2011. Human sensorimotor learning: adaptation, skill, and beyond. Curr. Opin. Neurobiol. 21, 636-644.

Kroencke, D.C., Lynch, S.G., Denney, D.R., 2000. Fatigue in multiple sclerosis: relationship to depression, disability, and disease pattern. Mult. Scler. 6, 131-136.

Krupp, L.B., Alvarez, L.A., LaRocca, N.G., Scheinberg, L.C., 1988. Fatigue in Multiple Sclerosis. Arch. Neurol. 45, 435-437.

Krupp, L.B., Coyle, P.K., Doscher, C., Miller, A., Cross, A.H., Jandorf, L., Halper, J., Johnson, B., Morgante, L., Grimson, R., 1995. Fatigue therapy in multiple sclerosis: Results of a double-blind, randomized, parallel trial of amantadine, pemoline, and placebo. Neurology 45, 1956-1961.

Krupp, L.B., LaRocca, N.G., Muir-Nash, J., Steinberg, A.D., 1989. The fatigue severity scale: Application to patients with multiple sclerosis and systemic lupus erythematosus. Arch. Neurol. 46, 1121-1123.

Krupp, L.B., Serafin, D.J., Christodoulou, C., 2010. Multiple sclerosis-associated fatigue. Expert Rev. Neurother. 10, 1437-1447.

Kuo, H.-I., Bikson, M., Datta, A., Minhas, P., Paulus, W., Kuo, M.-F., Nitsche, M.A., 2013. Comparing cortical plasticity induced by conventional and high-definition $4 \times 1$ ring tDCS: a neurophysiological study. Brain Stimul. 6, 644-648.

Kuo, M.-F., Nitsche, M.A., 2012. Effects of Transcranial Electrical Stimulation on Cognition. Clin. EEG Neurosci. $43,192-199$.

Kuo, M.-F., Paulus, W., Nitsche, M.A., 2013. Therapeutic effects of non-invasive brain stimulation with direct currents (tDCS) in neuropsychiatric diseases. Neuroimage 85, Part 3, 948-960. 
Kuo, M.-F., Unger, M., Liebetanz, D., Lang, N., Tergau, F., Paulus, W., Nitsche, M.A., 2008. Limited impact of homeostatic plasticity on motor learning in humans. Neuropsychologia 46, 2122-2128.

Lange, R., Volkmer, M., Heesen, C., Liepert, J., 2009. Modafinil effects in multiple sclerosis patients with fatigue. J. Neurol. 256, 645-650.

Lemieux, L., Allen, P.J., Franconi, F., Symms, M.R., Fish, D.R., 1997. Recording of EEG during fMRI experiments: patient safety. Magn. Reson. Med. 38, 943-952.

Leocani, L., Colombo, B., Magnani, G., Martinelli-Boneschi, F., Cursi, M., Rossi, P., Martinelli, V., Comi, G., 2001. Fatigue in multiple sclerosis is associated with abnormal cortical activation to voluntary movement--EEG evidence. Neuroimage 13, 1186-1192.

Lerdal, A., Celius, E.G., Krupp, L.B., Dahl, A.A., 2007. A prospective study of patterns of fatigue in multiple sclerosis. Eur. J. Neurol. 14, 1338-1343.

Liebetanz, D., Nitsche, M.A., Tergau, F., Paulus, W., 2002. Pharmacological approach to the mechanisms of transcranial DC-stimulation-induced after-effects of human motor cortex excitability. Brain 125, 2238-2247.

Lin, P.T., Hallett, M., 2009. The Pathophysiology of Focal Hand Dystonia. J. Hand Ther. 22, 109-114.

Lindenberg, R., Renga, V., Zhu, L.L., Nair, D., Schlaug, G., 2010. Bihemispheric brain stimulation facilitates motor recovery in chronic stroke patients. Neurol. 75, 2176-2184.

Lledo, P.-M., Alonso, M., Grubb, M.S., 2006. Adult neurogenesis and functional plasticity in neuronal circuits. Nat. Rev. Neurosci. 7, 179-93.

Lynch, M.A., 2004. Long-Term Potentiation and Memory. Physiol. Rev. 84, 87-136.

Mainero, C., Caramia, F., Pozzilli, C., Pisani, A., Pestalozza, I., Borriello, G., Bozzao, L., Pantano, P., 2004. fMRI evidence of brain reorganization during attention and memory tasks in multiple sclerosis. Neuroimage 21, 858-867.

Mainero, C., Faroni, J., Gasperini, C., Filippi, M., Giugni, E., Ciccarelli, O., Rovaris, M., Bastianello, S., Comi, G., Pozzilli, C., 1999. Fatigue and magnetic resonance imaging activity in multiple sclerosis. J. Neurol. 246, 454-458.

Manenti, R., Brambilla, M., Petesi, M., Ferrari, C., Cotelli, M., 2013. Enhancing verbal episodic memory in older and young subjects after non-invasive brain stimulation. Front. Aging Neurosci. 5,49 .

Martin, D.M., Alonzo, A., Mitchell, P.B., Sachdev, P., Gálvez, V., Loo, C.K., 2011. Fronto-extracephalic transcranial direct current stimulation as a treatment for major depression: An open-label pilot study. J. Affect. Disord. 134, 459-463.

Martin, S., Grimwood, P.D., Morris, R.G.M., 200o. Synaptic plasticity and memory: an evaluation of the hypothesis. Annu. Rev. Neurosci. 23, 649-711.

May, A., Gaser, C., 2006. Magnetic resonance-based morphometry: a window into structural plasticity of the brain. Curr. Opin. Neurol. 19, 407-411.

Meesen, R.L.J., Thijs, H., Leenus, D.J.F., Cuypers, K., 2013. A single session of $1 \mathrm{~mA}$ anodal tDCSsupported motor training does not improve motor performance in patients with multiple sclerosis. Restor. Neurol. Neurosci. (in press).

Miller, D.H., Barkhof, F., Frank, J.A., Parker, G.J.M., Thompson, A.J., 2002. Measurement of atrophy in multiple sclerosis: pathological basis, methodological aspects and clinical relevance. Brain 125, $1676-1695$. 
Mills, R.J., Young, C.A., Nicholas, R.S., Pallant, J.F., Tennant, A., 2009. Rasch analysis of the Fatigue Severity Scale in multiple sclerosis. Mult. Scler. 15, 81-87.

Minhas, P., Bansal, V., Patel, J., Ho, J.S., Diaz, J., Datta, A., Bikson, M., 2010. Electrodes for highdefinition transcutaneous DC stimulation for applications in drug delivery and electrotherapy, including tDCS. J. Neurosci. Methods 190, 188-197.

Miranda, P.C., Mekonnen, A., Salvador, R., Ruffini, G., 2013. The electric field in the cortex during transcranial current stimulation. Neuroimage $70,48-58$.

Moliadze, V., Antal, A., Paulus, W., 2010. Electrode-distance dependent after-effects of transcranial direct and random noise stimulation with extracephalic reference electrodes. Clin. Neurophysiol. $121,2165-2171$.

Monte-Silva, K., Kuo, M.-F., Hessenthaler, S., Fresnoza, S., Liebetanz, D., Paulus, W., Nitsche, M.A., 2013. Induction of late LTP-like plasticity in the human motor cortex by repeated non-invasive brain stimulation. Brain Stimul. 1-9.

Mori, F., Codecà, C., Kusayanagi, H., Monteleone, F., Buttari, F., Fiore, S., Bernardi, G., Koch, G., Centonze, D., 2010. Effects of anodal transcranial direct current stimulation on chronic neuropathic pain in patients with multiple sclerosis. J. Pain 11, 436-442.

Mori, F., Nicoletti, C.G., Kusayanagi, H., Foti, C., Restivo, D.A., Marciani, M.G., Centonze, D., 2013. Transcranial direct current stimulation ameliorates tactile sensory deficit in multiple sclerosis. Brain Stimul. 6, 654-659.

Mulquiney, P.G., Hoy, K.E., Daskalakis, Z.J., Fitzgerald, P.B., 2011. Improving working memory: exploring the effect of transcranial random noise stimulation and transcranial direct current stimulation on the dorsolateral prefrontal cortex. Clin. Neurophysiol. 122, 2384-2389.

Multiple Sclerosis Council for Clinical Practice Guidelines, 1998. Fatigue and multiple sclerosis: evidence-based management strategies for fatigue in multiple sclerosis. Washington, DC Paralyzed Veterans Am.

Murphy, T.H., Corbett, D., 2009. Plasticity during stroke recovery: from synapse to behaviour. Nat Rev Neurosci 10, 861-872.

Nair, D.G., Renga, V., Lindenberg, R., Zhu, L., Schlaug, G., 2011. Optimizing recovery potential through simultaneous occupational therapy and non-invasive brain-stimulation using tDCS. Restor. Neurol. Neurosci. 29, 411-420.

Niepel, G., Tench, C.R., Morgan, P.S., Evangelou, N., Auer, D.P., Constantinescu, C.S., 2006. Deep gray matter and fatigue in MS: a T1 relaxation time study. J. Neurol. 253, 896-902.

Nitsche, M., Paulus, W., 2009. Noninvasive brain stimulation protocols in the treatment of epilepsy: Current state and perspectives. Neurotherapeutics 6, 244-250.

Nitsche, M.A., Boggio, P.S., Fregni, F., Pascual-Leone, A., 2009. Treatment of depression with transcranial direct current stimulation (tDCS): A Review. Exp. Neurol. 219, 14-19.

Nitsche, M.A., Cohen, L.G., Wassermann, E.M., Priori, A., Lang, N., Antal, A., Paulus, W., Hummel, F., Boggio, P.S., Fregni, F., Pascual-Leone, A., 2008. Transcranial direct current stimulation: State of the art 2008. Brain Stimul. 1, 206-223.

Nitsche, M.A., Doemkes, S., Karaköse, T., Antal, A., Liebetanz, D., Lang, N., Tergau, F., Paulus, W., 2007. Shaping the effects of transcranial direct current stimulation of the human motor cortex. J. Neurophysiol. 97, 3109-3117. 
Nitsche, M.A., Fricke, K., Henschke, U., Schlitterlau, A., Liebetanz, D., Lang, N., Henning, S., Tergau, F., Paulus, W., 2003a. Pharmacological modulation of cortical excitability shifts induced by transcranial direct current stimulation in humans. J. Physiol. 553, 293-301.

Nitsche, M.A., Paulus, W., 2000. Excitability changes induced in the human motor cortex by weak transcranial direct current stimulation. J. Physiol. 527 Pt 3, 633-639.

Nitsche, M.A., Paulus, W., 2001. Sustained excitability elevations induced by transcranial DC motor cortex stimulation in humans. Neurol. 57, 1899-1901.

Nitsche, M.A., Paulus, W., 2011. Transcranial direct current stimulation - update 2011. Restor. Neurol. Neurosci. 29, 463-492.

Nitsche, M.A., Schauenburg, A., Lang, N., Liebetanz, D., Exner, C., Paulus, W., Tergau, F., 2003b. Facilitation of implicit motor learning by weak transcranial direct current stimulation of the primary motor cortex in the human. J. Cogn. Neurosci. 15, 619-626.

Opitz, A., Windhoff, M., Heidemann, R.M., Turner, R., Thielscher, A., 2011. How the brain tissue shapes the electric field induced by transcranial magnetic stimulation. Neuroimage $58,849-859$.

Orban de Xivry, J.-J., Marko, M.K., Pekny, S.E., Pastor, D., Izawa, J., Celnik, P., Shadmehr, R., 2011. Stimulation of the human motor cortex alters generalization patterns of motor learning. J. Neurosci. 31, 7102-7110.

Pardini, M., Bonzano, L., Mancardi, G.L., Roccatagliata, L., 2010. Frontal networks play a role in fatigue perception in multiple sclerosis. Behav. Neurosci. 124, 329-336.

Pascual-Leone, a, Torres, F., 1993. Plasticity of the sensorimotor cortex representation of the reading finger in Braille readers. Brain 116, 39-52.

Pascual-Leone, A., Freitas, C., Oberman, L., Horvath, J., Halko, M., Eldaief, M., Bashir, S., Vernet, M., Shafi, M., Westover, B., Vahabzadeh-Hagh, A., Rotenberg, A., 2011. Characterizing Brain Cortical Plasticity and Network Dynamics Across the Age-Span in Health and Disease with TMS-EEG and TMS-fMRI. Brain Topogr. 24, 302-315.

Pellicano, C., Gallo, A., Li, X., Ikonomidou, V.N., Evangelou, I.E., Ohayon, J.M., Stern, S.K., Ehrmantraut, M., Cantor, F., McFarland, H.F., Bagnato, F., 2010. Relationship of cortical atrophy to fatigue in patients with multiple sclerosis. Arch. Neurol. 67, 447-453.

Peña-Gómez, C., Sala-Lonch, R., Junqué, C., Clemente, I.C., Vidal, D., Bargalló, N., Falcón, C., VallsSolé, J., Pascual-Leone, Á., Bartrés-Faz, D., 2012. Modulation of large-scale brain networks by transcranial direct current stimulation evidenced by resting-state functional MRI. Brain Stimul. $5,252-263$.

Penner, I.-K., Rausch, M., Kappos, L., Opwis, K., Radü, E.W., 2003. Analysis of impairment related functional architecture in MS patients during performance of different attention tasks. J. Neurol. 250, 461-472.

Pirulli, C., Fertonani, A., Miniussi, C., 2013. The role of timing in the induction of neuromodulation in perceptual learning by transcranial electric stimulation. Brain Stimul. 6, 683-689.

Polanía, R., Paulus, W., Antal, A., Nitsche, M.A., 2011. Introducing graph theory to track for neuroplastic alterations in the resting human brain: a transcranial direct current stimulation study. Neuroimage 54, 2287-2296.

Polanía, R., Paulus, W., Nitsche, M.A., 2012a. Modulating cortico-striatal and thalamo-cortical functional connectivity with transcranial direct current stimulation. Hum. Brain Mapp. 33, 2499-2508. 
Polanía, R., Paulus, W., Nitsche, M.A., 2012b. Reorganizing the intrinsic functional architecture of the human primary motor cortex during rest with non-invasive cortical stimulation. PLoS One 7, e30971.

Polman, C.H., Reingold, S.C., Banwell, B., Clanet, M., Cohen, J. a, Filippi, M., Fujihara, K., Havrdova, E., Hutchinson, M., Kappos, L., Lublin, F.D., Montalban, X., O'Connor, P., Sandberg-Wollheim, M., Thompson, A.J., Waubant, E., Weinshenker, B., Wolinsky, J.S., 2011. Diagnostic criteria for multiple sclerosis: 2010 revisions to the McDonald criteria. Ann. Neurol. 69, 292-302.

Pugliatti, M., Rosati, G., Carton, H., Riise, T., Drulovic, J., Vécsei, L., Milanov, I., 2006. The epidemiology of multiple sclerosis in Europe. Eur. J. Neurol. 13, 700-722.

Radman, T., Ramos, R.L., Brumberg, J.C., Bikson, M., 2009. Role of cortical cell type and morphology in subthreshold and suprathreshold uniform electric field stimulation in vitro. Brain Stimul. 2, $215-228$.

Rammohan, K.W., Rosenberg, J.H., Lynn, D.J., Blumenfeld, A.M., Pollak, C.P., Nagaraja, H.N., 2002. Efficacy and safety of modafinil (Provigil $\AA$ ) for the treatment of fatigue in multiple sclerosis: a two centre phase 2 study. J. Neurol. Neurosurg. Psychiatry 72 , 179-183.

Reis, J., Fritsch, B., 2011. Modulation of motor performance and motor learning by transcranial direct current stimulation. Curr. Opin. Neurol. 24, 590-596.

Reis, J., Schambra, H.M., Cohen, L.G., Buch, E.R., Fritsch, B., Zarahn, E., Celnik, P.A., Krakauer, J.W., 2009. Noninvasive cortical stimulation enhances motor skill acquisition over multiple days through an effect on consolidation. Proc. Natl. Acad. Sci. U. S. A. 106, 1590-1595.

Riberto, M., Alfieri, F.M., Pacheco, K.M. de B., Leite, V.D., Kaihami, H.N., Fregni, F., Battistella, L.R., 2011. Efficacy of transcranial direct current stimulation coupled with a multidisciplinary rehabilitation program for the treatment of fibromyalgia. Open Rheumatol. J. 5, 45-50.

Riccitelli, G., Rocca, M.A., Forn, C., Colombo, B., Comi, G., Filippi, M., 2011a. Voxelwise assessment of the regional distribution of damage in the brains of patients with multiple sclerosis and fatigue. AJNR. Am. J. Neuroradiol. 32, 874-479.

Riccitelli, G., Rocca, M.A., Pagani, E., Rodegher, M.E., Rossi, P., Falini, A., Comi, G., Filippi, M., 2011b. Cognitive impairment in multiple sclerosis is associated to different patterns of gray matter atrophy according to clinical phenotype. Hum. Brain Mapp. 32, 1535-1543.

Roelcke, U., Kappos, L., Lechner-Scott, J., Brunnschweiler, H., Huber, S., Ammann, W., Plohmann, A., Dellas, S., Maguire, R.P., Missimer, J., Radü, E.W., Steck, A., Leenders, K.L., Radii, E.W., 1997. Reduced glucose metabolism in the frontal cortex and basal ganglia of multiple sclerosis patients with fatigue: A 18F-fluorodeoxyglucose positron emission tomography study. Neurology 48, $1566-1571$.

Roosendaal, S.D., Geurts, J.J.., Vrenken, H., Hulst, H.E., Cover, K.S., Castelijns, J.A., Pouwels, P.J.., Barkhof, F., 2009. Regional DTI differences in multiple sclerosis patients. Neuroimage 44, 1397403.

Rothwell, J.C., 1993. Evoked potentials, magnetic stimulation studies, and event-related potentials. Curr. Opin. Neurol. 6, 715-723.

Rueger, M.A., Keuters, M.H., Walberer, M., Braun, R., Klein, R., Sparing, R., Fink, G.R., Graf, R., Schroeter, M., 2012. Multi-Session Transcranial Direct Current Stimulation (tDCS) Elicits Inflammatory and Regenerative Processes in the Rat Brain. PLoS One 7, e43776.

Ruffini, G., Wendling, F., Merlet, I., Molaee-Ardekani, B., Mekonnen, A., Salvador, R., Soria-Frisch, A., Grau, C., Dunne, S., Miranda, P.C., 2013. Transcranial Current Brain Stimulation (tCS): Models and Technologies. Neural Syst. Rehabil. Eng. IEEE Trans. 21, 333-345. 
Ruohonen, J., Karhu, J., 2012. tDCS possibly stimulates glial cells. Clin. Neurophysiol. 123, 20062009.

Saab, C.Y., 2012. Pain-related changes in the brain: diagnostic and therapeutic potentials. Trends Neurosci. 35, 629-637.

Saiote, C., Goldschmidt, T., Timäus, C., Steenwijk, M.D., Opitz, A., Antal, A., Paulus, W., Nitsche, M.A., 2014. Impact of transcranial direct current stimulation on fatigue in multiple sclerosis. Restor. Neurol. Neurosci. (in press).

Saiote, C., Polanía, R., Rosenberger, K., Paulus, W., Antal, A., 2013a. High-Frequency TRNS Reduces BOLD Activity during Visuomotor Learning. PLoS One 8, e59669.

Saiote, C., Turi, Z., Paulus, W., Antal, A., 2013b. Combining functional magnetic resonance imaging with transcranial electrical stimulation. Front. Hum. Neurosci. 7, 435.

Salvador, R., Mekonnen, A., Ruffini, G., Miranda, P.C., 2010. Modeling the electric field induced in a high resolution realistic head model during transcranial current stimulation. Eng. Med. Biol. Soc. (EMBC), 2010 Annu. Int. Conf. IEEE.

Saucedo Marquez, C.M., Zhang, X., Swinnen, S.P., Meesen, R., Wenderoth, N., 2013. Task-specific effect of transcranial direct current stimulation on motor learning. Front. Hum. Neurosci. 7, 333.

Schambra, H.M., Abe, M., Luckenbaugh, D. a, Reis, J., Krakauer, J.W., Cohen, L.G., 2011. Probing for hemispheric specialization for motor skill learning: a transcranial direct current stimulation study. J. Neurophysiol. 106, 652-661.

Schoonheim, M.M., Geurts, J.J.G., Landi, D., Douw, L., van der Meer, M.L., Vrenken, H., Polman, C.H., Barkhof, F., Stam, C.J., 2013. Functional connectivity changes in multiple sclerosis patients: a graph analytical study of MEG resting state data. Hum. Brain Mapp. 34, 52-61.

Schwid, S., Petrie, M., Murray, R., Leitch, J., Bowen, J., Alquist, A., Pelligrino, R., Roberts, A., HarperBennie, J., Milan, M., Guisado, R., Luna, B., Montgomery, L., Lamparter, R., Ku, Y., Lee, H., Goldwater, D., Cutter, G., Webbon, B., Group, N.C.S., 2003. A randomized controlled study of the acute and chronic effects of cooling therapy for MS. Neurology 60, 1955-1960.

Sepulcre, J., Masdeu, J.C., Goñi, J., Arrondo, G., Vélez de Mendizábal, N., Bejarano, B., Villoslada, P., 2009. Fatigue in multiple sclerosis is associated with the disruption of frontal and parietal pathways. Mult. Scler. 15, 337-344.

Shekhawat, G.S., Stinear, C.M., Searchfield, G.D., 2013. Transcranial Direct Current Stimulation Intensity and Duration Effects on Tinnitus Suppression. Neurorehabilitation and Neural Repair $27,164-172$.

Smith, S.M., 2002. Fast robust automated brain extraction. Hum. Brain Mapp. 17, 143-155.

Smith, S.M., Zhang, Y., Jenkinson, M., Chen, J., Matthews, P.M., Federico, A., De Stefano, N., 2002. Accurate, Robust, and Automated Longitudinal and Cross-Sectional Brain Change Analysis. Neuroimage 17, 479-489.

Snowball, A., Tachtsidis, I., Popescu, T., Thompson, J., Delazer, M., Zamarian, L., Zhu, T., Cohen Kadosh, R., 2013. Long-term enhancement of brain function and cognition using cognitive training and brain stimulation. Curr. Biol. 23, 987-992.

Stagg, C.J., Jayaram, G., Pastor, D., Kincses, Z.T., Matthews, P.M., Johansen-Berg, H., 2011. Polarity and timing-dependent effects of transcranial direct current stimulation in explicit motor learning. Neuropsychologia 49, 800-804.

Stagg, C.J., Johansen-Berg, H., 2013. Studying the Effects of Transcranial Direct-Current Stimulation in Stroke Recovery Using Magnetic Resonance Imaging. Front. Hum. Neurosci. 7, 857. 
Stagg, C.J., O’Shea, J., Kincses, Z.T., Woolrich, M., Matthews, P.M., Johansen-Berg, H., 2009. Modulation of movement-associated cortical activation by transcranial direct current stimulation. Eur. J. Neurosci. 30, 1412-1423.

Stam, C.J., Jones, B.F., Nolte, G., Breakspear, M., Scheltens, P., 2007. Small-world networks and functional connectivity in Alzheimer's disease. Cereb. Cortex 17, 92-99.

Stam, C.J., Reijneveld, J.C., 2007. Graph theoretical analysis of complex networks in the brain. Nonlinear Biomed. Phys. 1.

Stankoff, B., Waubant, E., Confavreux, C., Edan, G., Debouverie, M., Rumbach, L., Moreau, T., Pelletier, J., Lubetzki, C., Clanet, M., Group, F.M.S., 2005. Modafinil for fatigue in MS: A randomized placebo-controlled double-blind study Neurol. $64,1139-1143$.

Steenwijk, M.D., Pouwels, P.J.W., Daams, M., van Dalen, J.W., Caan, M.W. a., Richard, E., Barkhof, F., Vrenken, H., 2013. Accurate white matter lesion segmentation by $\mathrm{k}$ nearest neighbor classification with tissue type priors (kNN-TTPs). NeuroImage Clin. 3, 462-469.

Steinhoff, B.J., Tumani, H., Otto, M., Mursch, K., Wiltfang, J., Herrendorf, G., Bittermann, H.-J., Felgenhauer, K., Paulus, W., Markakis, E., 1999. Cisternal S100 protein and neuron-specific enolase are elevated and site-specific markers in intractable temporal lobe epilepsy. Epilepsy Res. $36,75-82$.

Stephan, K.E., Baldeweg, T., Friston, K.J., 2006. Synaptic plasticity and dysconnection in schizophrenia. Biol. Psychiatry 59, 929-939.

Tartaglia, M.C., Narayanan, S., Francis, S.J., Santos, A.C., De Stefano, N., Lapierre, Y., Arnold, D.L., 2004. The relationship between diffuse axonal damage and fatigue in multiple sclerosis. Arch. Neurol. 61, 201-207.

Tedeschi, G., Dinacci, D., Lavorgna, L., Prinster, A., Savettieri, G., Quattrone, A., Livrea, P., Messina, C., Reggio, A., Servillo, G., Bresciamorra, V., Orefice, G., Paciello, M., Brunetti, A., Paolillo, A., Coniglio, G., Bonavita, S., Di Costanzo, A., Bellacosa, A., Valentino, P., Quarantelli, M., Patti, F., Salemi, G., Cammarata, E., Simone, I., Salvatore, M., Bonavita, V., Alfano, B., 2007. Correlation between fatigue and brain atrophy and lesion load in multiple sclerosis patients independent of disability. J. Neurol. Sci. 263, 15-19.

Téllez, N., Alonso, J., Río, J., Tintoré, M., Nos, C., Montalban, X., Rovira, A., 2008. The basal ganglia: a substrate for fatigue in multiple sclerosis. Neuroradiology 50, 17-23.

Teo, F., Hoy, K.E., Daskalakis, Z.J., Fitzgerald, P.B., 2011. Investigating the Role of Current Strength in tDCS Modulation of Working Memory Performance in Healthy Controls. Front. Psychiatry 2, 45.

Terney, D., Chaieb, L., Moliadze, V., Antal, A., Paulus, W., 2008. Increasing human brain excitability by transcranial high-frequency random noise stimulation. J. Neurosci. 28, 14147-14155.

Thickbroom, G.W., Byrnes, M.L., Archer, S.A., Kermode, A.G., Mastaglia, F.L., 2005. Corticomotor organisation and motor function in multiple sclerosis. J. Neurol. 252, 765-771.

Tomassini, V., Jbabdi, S., Kincses, Z.T., Bosnell, R., Douaud, G., Pozzilli, C., Matthews, P.M., Johansen-Berg, H., 2011. Structural and functional bases for individual differences in motor learning. Hum. Brain Mapp. 32, 494-508.

Valle, A., Roizenblatt, S., Botte, S., Zaghi, S., Riberto, M., Tufik, S., Boggio, P.S., Fre, 2009. Efficacy of anodal transcranial direct current stimulation (tDCS) for the treatment of fibromyalgia: results of a randomized, sham-controlled longitudinal clinical. J. Pain Manag. 2, 353-361.

Van der Werf, S.P., Jongen, P.J.H., Lycklama à Nijeholt, G.J., Barkhof, F., Hommes, O.R., Bleijenberg, G., 1998. Fatigue in multiple sclerosis: Interrelations between fatigue complaints, cerebral MRI abnormalities and neurological disability. J. Neurol. Sci. 160, 164-170. 
Van Kessel, K., Moss-Morris, R., Willoughby, E., Chalder, T., Johnson, M.H., Robinson, E., 2008. A Randomized Controlled Trial of Cognitive Behavior Therapy for Multiple Sclerosis Fatigue. Psychosom. Med. 70 , 205-213.

Vines, B.W., Cerruti, C., Schlaug, G., 2008. Dual-hemisphere tDCS facilitates greater improvements for healthy subjects' non-dominant hand compared to uni-hemisphere stimulation. BMC Neurosci. 9, 103.

Vogt, B.A., Finch, D.M., Olson, C.R., 1992. Functional Heterogeneity in Cingulate Cortex: The Anterior Executive and Posterior Evaluative Regions. Cereb. Cortex 2, 435-443.

Wassermann, E.M., Zimmermann, T., 2012. Transcranial magnetic brain stimulation: Therapeutic promises and scientific gaps. Pharmacol. Ther. 133, 98-107.

Wattjes, M.P., Lutterbey, G.G., Gieseke, J., Träber, F., Klotz, L., Schmidt, S., Schild, H.H., 2007. Double Inversion Recovery Brain Imaging at 3T: Diagnostic Value in the Detection of Multiple Sclerosis Lesions. Am. J. Neuroradiol. 28, 54-59.

Whitlock, J.R., Heynen, A.J., Shuler, M.G., Bear, M.F., 2006. Learning induces long-term potentiation in the hippocampus. Science 313, 1093-1097.

Windhoff, M., Opitz, A., Thielscher, A., 2013. Electric field calculations in brain stimulation based on finite elements: An optimized processing pipeline for the generation and usage of accurate individual head models. Hum. Brain Mapp. 34, 923-935.

Woolrich, M.W., Ripley, B.D., Brady, M., Smith, S.M., 2001. Temporal Autocorrelation in Univariate Linear Modeling of FMRI Data. Neuroimage 14, 1370-1386.

Yaldizli, Ö., Glassl, S., Sturm, D., Papadopoulou, A., Gass, A., Tettenborn, B., Putzki, N., 2011. Fatigue and progression of corpus callosum atrophy in multiple sclerosis. J. Neurol. 258, 2199-2205.

Yaldizli, Ö., Penner, I.-K., Frontzek, K., Naegelin, Y., Amann, M., Papadopoulou, A., Sprenger, T., Kuhle, J., Calabrese, P., Radü, E.W., Kappos, L., Gass, A., 2013. The relationship between total and regional corpus callosum atrophy, cognitive impairment and fatigue in multiple sclerosis patients. Mult. Scler. J. (in press).

Zatorre, R.J., Fields, R.D., Johansen-Berg, H., 2012. Plasticity in gray and white: neuroimaging changes in brain structure during learning. Nat. Neurosci. 15, 528-536.

Zellini, F., Niepel, G., Tench, C.R., Constantinescu, C.S., 2009. Hypothalamic involvement assessed by T1 relaxation time in patients with relapsing-remitting multiple sclerosis. Mult. Scler. $15,1442-$ 1449. 


\section{List of Abbreviations}

ACC - anterior cingulate cortex

ANOVA - analysis of variance

BDI - Beck’s Depression Inventory

BOLD - Blood Oxygen Level Dependent

$\mathrm{ClF}$ - contralateral forehead

CNS - Central Nervous System

CSF - cerebrospinal fluid

DC - direct current

DLPFC - dorsolateral prefrontal cortex

DMN - default mode network

DTI - diffusion tensor imaging

EDSS - Expanded Disability Status Scale

EEG - electroencephalogram

EV - explanatory variable

FA - fractional anisotropy

FEAT - FMRI Expert Analysis Tool

FLAIR - fluid-attenuated inversion-

recovery

FLASH - fast low angle shot

FLIRT - FMRIB's Linear Image

Registration Tool

fMRI - functional magnetic resonance imaging

FNIRT - FMRIB's Non-linear Image

Registration Tool

FSL - FMRIB’s Software Library

FSS - Fatigue Severity Scale

FWHM - Full-Width Half-Maximum
GLM - general linear model

GM - gray matter

HADS-A - Hamilton's Anxiety and

Depression Scale - Anxiety

HADS-D - Hamilton's Anxiety and

Depression Scale - Depression

Hf-tRNS - high-frequency transcranial random noise stimulation

HPA - hypothalamo-pituitary-adrenal

HRF - hemodynamic response function

ICA - independent component analysis

IFN- $\gamma$ - Interferon- $\gamma$

IL-6 - Interleukin-6

Lf-tRNS - low-frequency transcranial random noise stimulation

LOC - lateral occipital cortex

LTD - long-term potentiation

LTP - long-term depression

$\mathrm{LV}$ - lesion volume

M1 - primary motor cortex

MEP - motor evoked potential

MFIS - Modified Fatigue Impact Scale

MNI - Montreal National Institute

MRI - magnetic resonance imaging

MS - multiple sclerosis

MSFSS - Multiple Sclerosis-specific

Fatigue Severity Scale

$\mathrm{NAA} / \mathrm{Cr}$ - N-acetyl aspartate/Creatine

Ratio 
NIBS - non-invasive brain stimulation

NMDA - N-Methyl-D-aspartate

NSE - neuron-specific enolase

PCC - posterior cingulate cortex

PET - positron emission tomography

PMd - dorsal premotor cortex

PPC - posterior parietal cortex

PPMS - primary progressive MS

PRMS - progressive-relapsing MS

ROI - region of interest

RRMS - relapsing-remitting MS

SEM - standard error of mean

SM1 - primary somatosensory cortex

SMA - supplementary motor area

SNR - signal-to-noise ratio

SPMS - secondary progressive MS
SRTT - serial reaction time task

tACS - transcranial alternating current

stimulation

tDCS - transcranial direct current

stimulation

TE - echo-time

tES - transcranial electric stimulation

TI - inversion time

TMS - transcranial magnetic stimulation

TNF- $\alpha$ - tumour necrosis factor- $\alpha$

$\mathrm{TR}$ - repetition time

tRNS - transcranial random noise

stimulation

$\mathrm{V} 1$ - primary visual cortex

VAS - visual analogy scale

$\mathrm{WM}$ - white matter 


\section{Contributions}

In Project 1, Konstantin Rosenberger contributed to data acquisition and Rafael Polanía contributed to data acquisition and analysis.

In Project 2, Klara Boros, Thomas Goldschmidt and Charles Timäus contributed to data acquisition; Martijn Steenwijk (Dept. of Radiology and Nuclear Medicine, VU Medical Center, Amsterdam) performed the automatic lesion segmentation, Thomas Goldschmidt contributed to manual correction of lesion masks; Wiebke Albrecht contributed to the MRI analysis; Alexander Opitz performed the electric field simulation. Prof. Nitsche contributed to data analysis.

All MRI sessions were performed in the Dept. of Cognitive Neurology (UMG). 


\section{Acknowledgements}

First of all, I would like to thank Prof. Antal for the support, encouragement and greatly appreciated patience; without her this thesis would not have been possible. I am also very thankful to Prof. Michael Hörner and Prof. Florentin Wörgötter, for their guidance throughout my $\mathrm{PhD}$ and valuable help in the toughest times. I would also like to thank Prof. Paulus, for the opportunity to work for my $\mathrm{PhD}$ in his department and for the ceaseless encouragement in making the research projects move forward. I am immensely grateful to Prof. Michael Nitsche for all the support and advice, and pleasant scientific discussions.

I would like to express sincere gratitude to Marion Kurze for helping with all the bureaucratic issues with so much patience and sympathy. I am also very thankful to the medical and technical staff of the department of Clinical Neurophysiology, for assistance with the patients and in several other ways. I am very grateful to Peter Dechent, Carsten Schmidt-Samoa, Gunter Helms, Ilona Pfahlert, Britta Perl and Nina Kaeding from the department of Cognitive Neurology, for MRI advice and assistance, and for the friendly and welcoming environment.

I would also like to acknowledge my colleagues at the department for lending their help in numerous situations and for creating a pleasant and enthusiastic environment for everyday work: Rafael Polanía, Konstantin Rosenberger, José Casadiego Bastidas, Geza Ambrus, Olga Gamboa, Manuel Hewitt, Shane Fresnoza, Giorgi Batsikadze, Thomas Goldschmidt, Charles Timäus, Zsolt Turi and Alexander Opitz.

My thanks go also to the GGNB and CSN for developing a program that supports $\mathrm{PhD}$ students in many ways and for fostering creative and interactive scientific environment. My special thanks go to Kirsten Pöhlker for so nicely welcoming me to Göttingen and for all the friendly assistance.

Heartfelt thanks go to my friends: to Giorgi and João for all the laughs and bearing with me through difficult periods; to Daniela for her cheerfulness and support; to Sandra, who will make me miss Göttingen; and to Sara, Marta, Manel and Inês. My deepest gratitude goes to MM for the invaluable encouragement, advice, and strength to keep on going. I also thank my mother, father and brother, and remaining family for the unconditional support.

Last but not least, I would like to thank my previous teachers, particularly at Instituto Gregoriano de Lisboa, for advice and inspiration that helped become the person I am today. 


\section{CV}

Catarina Saiote Ferreira Leite

Hospitalstrasse 20

37073 Göttingen

Date of Birth: 15.05.1985

Nationality: Portuguese

\section{Education}

Since May 2010

PhD student in Systems Neuroscience at the University of Göttingen.

November $2009 \quad$ MSc in Biomedical Engineering at Instituto Superior Técnico, Technical University of Lisbon

September 2006 - $\quad$ Exchange student at Czech Technical University, Prague

February, 2007

\section{Work Experience}

December 2009 - April 2010:

Research assistant at the department of Physics, Instituto Superior Técnico, Technical University of Lisbon.

\section{Publications}

Saiote C, Polanía R, Rosenberger K, Paulus W, Antal A. (2013). High-frequency TRNS reduces $B O L D$ activity during visuomotor learning. PLoS One. (8)3.

Saiote, C, Turi, Z, Paulus, W, Antal, A. (2013). Combining functional magnetic resonance imaging with transcranial electrical stimulation. Front. Hum. Neurosci. (7)435.

Chaieb L, Antal A, Pisoni A, Saiote C, Opitz A, Ambrus GG, Focke N, Paulus W. (2013). Safety of $5 \mathrm{kHz}$ tACS. Brain Stimulation (in press).

Antal, A, Fischer, T, Miller, R, Saiote, C, Chaieb, L, Wang, DJJ, Plessow, F, Paulus, W, Kirschbaum, C. Transcranial electrical stimulation modifies the neuronal response to psychosocial stress exposure. Human Brain Mapping (accepted).

Saiote C, Goldschmidt T, Timäus C, Steenwijk M.D., Opitz A, Antal A, Paulus W, Nitsche M. Impact of transcranial direct current stimulation on fatigue in multiple sclerosis. (accepted). 
Chaieb L, Saiote C, Paulus W, Antal A. Effects of Transcranial Electrical Stimulation on Sensory Functions. In: Improving functions in the typical brain (Editor: C. Kadosh). Elsevier, 2014, in press

Posters and presentations (only first author)

Saiote, C, Silva, J, Gomes, C, Lauterbach, M, Reimão, S, Figueiredo, P. Parametric fMRI correlates of faces at multiple orientations, HBM 2010, Barcelona.

Saiote, C, Polanía P, Rosenberger K, Paulus, W, Antal, A. Evaluation of the effect of transcranial electrical stimulation on motor learning using fMRI, ECCN 2011, Rome (best poster award).

Saiote, C, Nemeth, D, Janacsek, K, Turi, Zs, Ambrus, G, Paulus, W, Antal, A. Cathodal transcranial direct current stimulation over the right prefrontal cortex improves implicit learning in healthy individuals. $5^{\text {th }}$ International Conference on Non-invasive Brain Stimulation 2013, Leipzig.

Saiote C, Goldschmidt T, Timäus C, Steenwijk MD, Opitz A, Antal A, Paulus W, Nitsche M. Impact of prefrontal anodal transcranial direct current stimulation on fatigue in multiple sclerosis. GGNB Science Day 2013, Göttingen. 